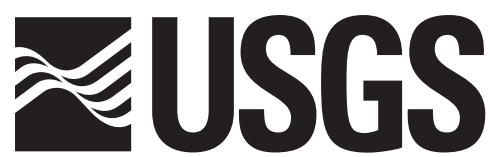

science for a changing world

In cooperation with the Texas Department of Transportation

\title{
Synthesis of Rainfall and Runoff Data Used for Texas Department of Transportation Research Projects 0-4193 and 0-4194
}

Open-File Report 2004-1035

(TxDOT Research Reports 0-4193-2 and 0-4194-2)

U.S. Department of the Interior

U.S. Geological Survey 


\begin{tabular}{|c|c|c|c|}
\hline $\begin{array}{l}\text { 1. Report No. } \\
\text { FHWA/TX-04/0-4193-2 } \\
\text { FHWA/TX-04/0-4194-2 }\end{array}$ & 2. Government Accession No. & \multicolumn{2}{|l|}{ 3. Recipient's Catalog No. } \\
\hline \multicolumn{2}{|c|}{$\begin{array}{l}\text { SYNTHESIS OF RAINFALL AND RUNOFF DATA USED } \\
\text { FOR TEXAS DEPARTMENT OF TRANSPORTATION } \\
\text { RESEARCH PROJECTS 0-4193 AND 0-4194 }\end{array}$} & \multicolumn{2}{|l|}{$\begin{array}{l}\text { 5. Report Date } \\
\text { June } 2004\end{array}$} \\
\hline \multicolumn{2}{|c|}{$\begin{array}{l}\text { 7. Author(s) } \\
\text { William H. Asquith, David B. Thompson, Theodore G. Cleveland, and } \\
\text { Xing Fang }\end{array}$} & \multicolumn{2}{|c|}{$\begin{array}{l}\text { 8. Performing Organization Report No. } \\
\text { OFR 2004-1035 }\end{array}$} \\
\hline \multicolumn{2}{|c|}{$\begin{array}{l}\text { 9. Performing Organization Name and Address } \\
\text { U.S. Geological Survey } \\
\text { Water Resources Division } \\
8027 \text { Exchange Drive } \\
\text { Austin, Texas } 78754\end{array}$} & \multicolumn{2}{|l|}{$\begin{array}{l}\text { 11. Contract or Grant No. } \\
\text { Project } 0-4193\end{array}$} \\
\hline \multicolumn{2}{|c|}{$\begin{array}{l}\text { 12. Sponsoring Agency Name and Address } \\
\text { Texas Department of Transportation } \\
\text { Research and Technology Implementation Office } \\
\text { 4000 Jackson Ave., Bldg. } 1 \\
\text { P.O. Box } 5080 \\
\text { Austin, TX } 78731\end{array}$} & \multicolumn{2}{|l|}{ 14. Sponsoring Agency Code } \\
\hline \multicolumn{4}{|c|}{$\begin{array}{l}\text { 15. Supplementary Notes } \\
\text { Project conducted in cooperation with the Te }\end{array}$} \\
\hline \multicolumn{4}{|c|}{$\begin{array}{l}\text { 16. Abstract } \\
\text { In the early 2000s, the Texas Department of Transportation funded several research projects to examine the unit } \\
\text { hydrograph and rainfall hyetograph techniques for hydrologic design in Texas for the estimation of design flows for } \\
\text { stormwater drainage systems. A research consortium comprised of Lamar University, Texas Tech University, the } \\
\text { University of Houston, and the U.S. Geological Survey (USGS), was chosen to examine the unit hydrograph and rainfall } \\
\text { hyetograph techniques. Rainfall and runoff data collected by the USGS at } 91 \text { streamflow-gaging stations in Texas formed a } \\
\text { basis for the research. These data were collected as part of USGS small-watershed projects and urban watershed studies that } \\
\text { began in the late } 1950 \text { s and continued through most of the } 1970 \text { s; a few gages were in operation in the mid-1980s. Selected } \\
\text { hydrologic events from these studies were available in the form of over } 220 \text { printed reports, which offered the best } \\
\text { aggregation of hydrologic data for the research objectives. Digital versions of the data did not exist. Therefore, a significant } \\
\text { effort was undertaken by the research consortium to manually enter the data into a digital database from the printed record. } \\
\text { The rainfall and runoff data for over } 1,650 \text { storms were entered. To enhance data integrity, considerable quality-control and } \\
\text { quality-assurance efforts were conducted as the database was assembled and after assembly to enhance data integrity. This } \\
\text { report documents the database and informs interested parties on its usage. }\end{array}$} \\
\hline $\begin{array}{l}\text { 17. Key Words } \\
\text { Precipitation, Rainfall, Runoff, } \\
\text { watershed, Texas }\end{array}$ & nyetograph, Unit hydrograph, Small & $\begin{array}{l}\text { 18. Distribution Statement } \\
\text { No restrictions. This documen } \\
\text { to the public through the Nati } \\
\text { Information Service, Springfi } \\
\text { 22161, www.ntis.gov }\end{array}$ & $\begin{array}{l}\text { is available } \\
\text { nal Technical } \\
d \text {, Virginia }\end{array}$ \\
\hline $\begin{array}{l}\text { 19. Security Classif. (of report) } \\
\text { Unclassified }\end{array}$ & $\begin{array}{l}\text { 20. Security Classif. (of this page) } \\
\text { Unclassified }\end{array}$ & $\begin{array}{l}\text { 21. No. of pages } \\
1,050\end{array}$ & 22. Price \\
\hline
\end{tabular}




\section{Synthesis of Rainfall and Runoff Data Used for Texas Department of Transportation Research Projects 0-4193 and 0-4194}

William H. Asquith, David B. Thompson, Theodore G. Cleveland, and Xing Fang

In cooperation with the Texas Department of Transportation

Open-File Report 2004-1035

(TxDOT Research Reports 0-4193-2 and 0-4194-2)

U.S. Department of the Interior U.S. Geological Survey 


\section{U.S. DEPARTMENT OF THE INTERIOR}

Gale A. Norton, Secretary

\section{U.S. GEOLOGICAL SURVEY}

Charles G. Groat, Director

Austin, Texas: June 2004

For more information about the USGS and its products:

Telephone: 1-888-ASK-USGS

World Wide Web: http://www.usgs.gov/

Any use of trade, product, or firm names is for descriptive purposes only and does not imply endorsement by the U.S. Government.

Although this report is in the public domain, permission must be secured from the individual copyright owners to reproduce any copyrighted materials contained within this report.

The contents of this report do not necessarily reflect the official view or policies of the Texas Department of Transportation (TxDOT). This report does not constitute a standard, specification, or regulation. 


\section{CONTENTS}

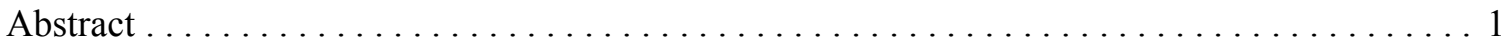

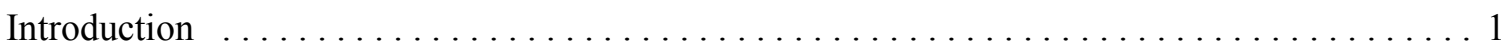

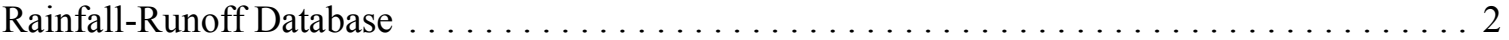

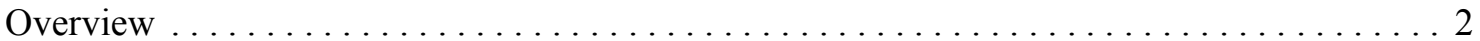

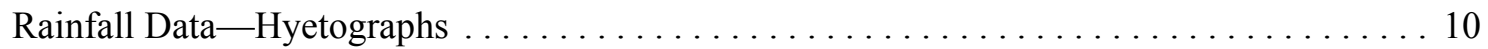

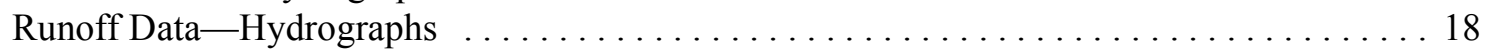

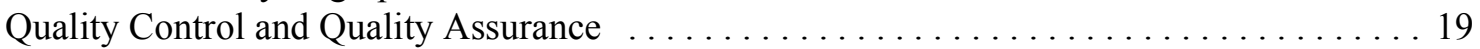

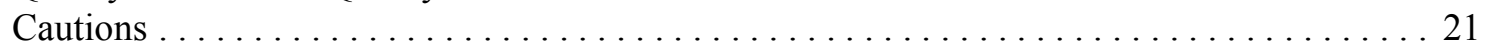

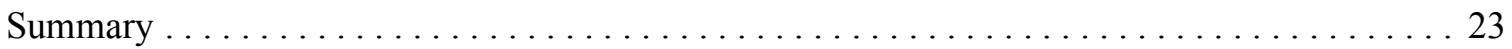

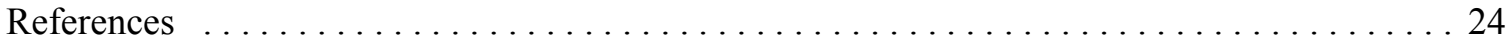

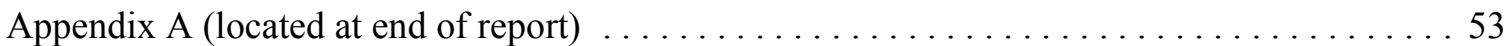

Hyetograph Data Files . . . . . . . . . . . . . . . . . . . . . . . . . . . . 53

austin Database Module ................................. 53

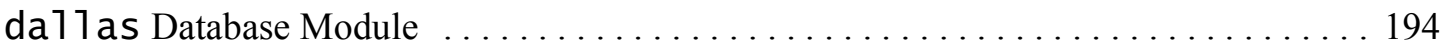

fortworth Database Module ... . . . . . . . . . . . . . . . . . . . . . . . . 285

sanantonio Database Module . . . . . . . . . . . . . . . . . . . . 342

sma 11 rura1sheds Database Module . . . . . . . . . . . . . . . 400

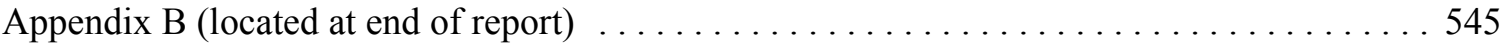

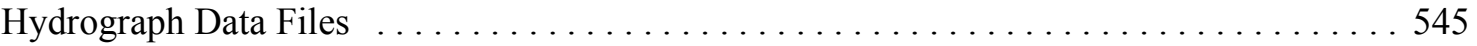

austin Database Module .................................... 545

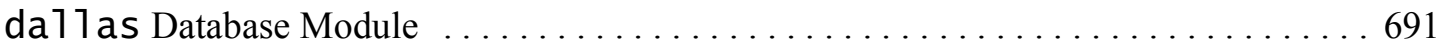

fortworth Database Module . . . . . . . . . . . . . . . . . . . . . . . . . . . . . . 776

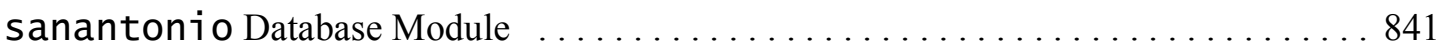

sma 11 rura1sheds Database Module ............................ 895

\section{FIGURES}

1. Map showing locations of USGS streamflow-gaging stations represented in the database . . . 2

2. Directory structure of the aust in database module $\ldots \ldots \ldots \ldots \ldots \ldots \ldots \ldots \ldots$

3. Directory structure of the da 11 as database module $\ldots \ldots \ldots \ldots \ldots \ldots \ldots \ldots$

4. Directory structure of the fortworth database module $\ldots \ldots \ldots \ldots \ldots \ldots \ldots \ldots$

5. Directory structure of the sanantonio database module $\ldots \ldots \ldots \ldots \ldots \ldots \ldots$

6. Directory structure of the sma 11 rura 1 sheds database module . . . . . . . . . . 10

7. Example hyetograph data file austin/shoa1creek/sta08156800_d/hyetos/

rain_sta08156800_1981_0523.dat from the austin database module . . . . . 11

8. Hyetographs for austin database module -401 storm events represented $\ldots \ldots \ldots \ldots 13$

9. Hyetographs for da 11 as database module -240 storm events represented . . . . . . . 14

10. Hyetographs for for tworth database module - 194 storm events represented . . . . . 15

11. Hyetographs for sanantonio database module -215 storm events represented . . . . 16

12. Hyetographs for sma 11 rura 1 sheds database module -607 storm events represented . 17

13. Example hydrograph data file austin/shoa1creek/sta08156800_d/hyetos/

unit_sta08156800_1981_0523.dat from the austin database module . . . . . 18

14. Location map for aust in database module adopted from

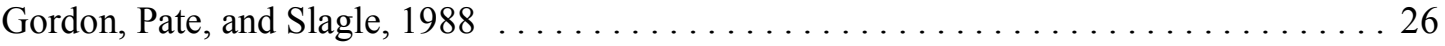

15. Location map for da 11 as database module adopted from

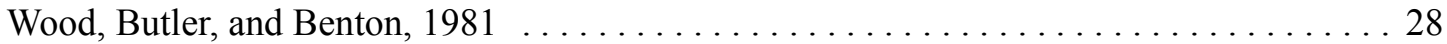

16. Location map for fortworth database module adopted from

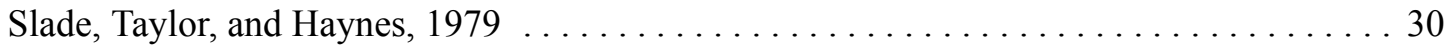

17. Location map for sanantonio database module adopted from Perez, 1983 . . . . . . . 31 
18. Location map for sma 11 rura 1 sheds database module for Cow Bayou watershed

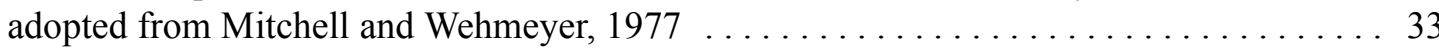

19. Location map for sma 11 rura 1 sheds database module for Green Creek watershed

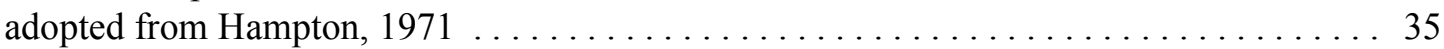

20. Location map for sma 11 rura 1 sheds database module for Little Pond and North Elm Creek watersheds adopted from Mitchell, $1974 \ldots \ldots \ldots \ldots \ldots \ldots \ldots$

21. Location map for sma11 rura1sheds database module for Deep Creek

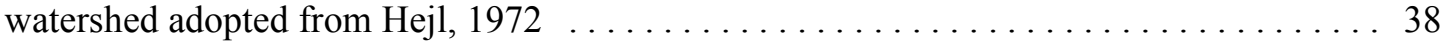

22. Location map for sma 17 rura 1 sheds database module for Mukewater Creek

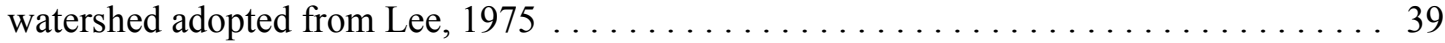

23. Location map for sma 11 rura 1 sheds database module for Calaveras Creek

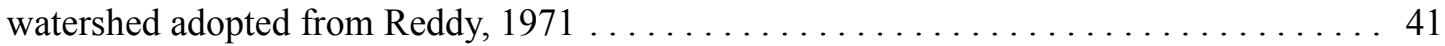

24. Location map for sma11 rura1sheds database module for Escondido Creek

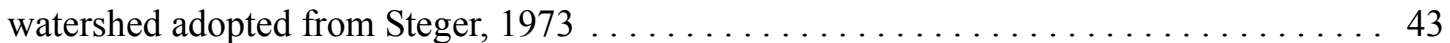

25. Location map for sma11 rura1sheds database module for Elm Fork Trinity River watershed adopted from Lucero, 1973 . . . . . . . . . . . . . . . . . . . . . . . . . . . . . 44

26. Location map for sma 11 rura 1 sheds database module for Honey Creek

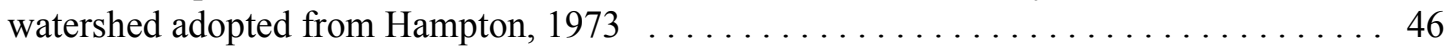

27. Location map for sma11 rura1 sheds database module for Little Elm Creek watershed adopted from Slade and Taylor, $1978 \ldots \ldots \ldots \ldots \ldots \ldots \ldots \ldots \ldots \ldots$

28. Location map for sma 11 rura1sheds database module for North Creek watershed adopted from Kidwell, 1979 . . . . . . . . . . . . . . . . . . . 50

29. Location map for sma 11 rura 1 sheds database module for Pin Oak Creek watershed adopted from Hampton, $1974 \ldots \ldots \ldots \ldots \ldots \ldots \ldots \ldots \ldots \ldots \ldots \ldots \ldots \ldots$

\section{TABLES}

1. U.S. Geological Survey streamflow-gaging stations within the austin database module . . 4

2. U.S. Geological Survey streamflow-gaging stations within the da11 as database module . . 5

3. U.S. Geological Survey streamflow-gaging stations within the fortworth

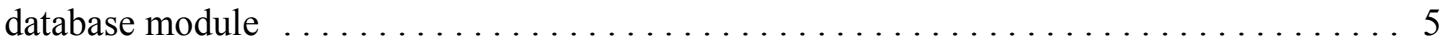

4. U.S. Geological Survey streamflow-gaging stations within the sanantonio

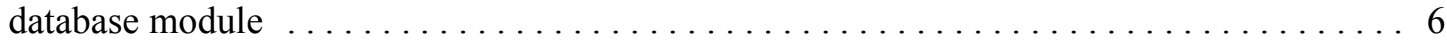

5. U.S. Geological Survey streamflow-gaging stations within the sma11 rura1sheds

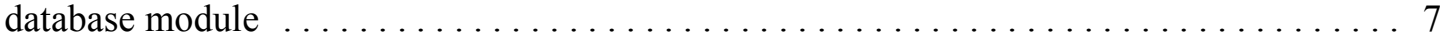

\section{ACKNOWLEDGMENTS}

The authors recognize the contributions of the following TxDOT personnel: George Herrmann, San Angelo District, Project Director 0-4193; Amy Ronnfeldt, Design Division (Austin), Project Director 0-4194; David Stolpa, Design Division (Austin), Program Coordinator for Projects 0-4193 and 0-4194. 


\title{
SYNTHESIS OF RAINFALL AND RUNOFF DATA USED FOR TEXAS DEPARTMENT OF TRANSPORTATION RESEARCH PROJECTS 0-4193 AND 0-4194
}

\author{
By William H. Asquith ${ }^{1}$, David B. Thompson ${ }^{2}$, \\ Theodore G. Cleveland ${ }^{3}$, and Xing Fang ${ }^{4}$
}

\begin{abstract}
In the early 2000s, the Texas Department of Transportation funded several research projects to examine the unit hydrograph and rainfall hyetograph techniques for hydrologic design in Texas for the estimation of design flows for stormwater drainage systems. A research consortium comprised of Lamar University, Texas Tech University, the University of Houston, and the U.S. Geological Survey (USGS), was chosen to examine the unit hydrograph and rainfall hyetograph techniques. Rainfall and runoff data collected by the USGS at 91 streamflow-gaging stations in Texas formed a basis for the research. These data were collected as part of USGS small-watershed projects and urban watershed studies that began in the late 1950s and continued through most of the 1970s; a few gages were in operation in the mid-1980s. Selected hydrologic events from these studies were available in the form of over 220 printed reports, which offered the best aggregation of hydrologic data for the research objectives. Digital versions of the data did not exist. Therefore, significant effort was undertaken by the consortium to manually enter the data into a digital database from the printed record. The rainfall and runoff data for over 1,650 storms were entered. To enhance data integrity, considerable quality-control and qualityassurance efforts were conducted as the database was assembled and after assembly to enhance data integrity. This report documents the database and informs interested parties on its usage.
\end{abstract}

\section{INTRODUCTION}

The U.S. Geological Survey (USGS) in collaborative peer-to-peer research projects with Lamar University, Texas Tech University, and the University of Houston, has developed a digital database of rainfall and runoff values for selected watersheds in Texas. The research was sponsored by Texas Department of Transportation research (TxDOT) projects $0-4139$ and $0-4194$. These projects are hereafter referred to as the "TxDOT research projects." The database is an aggregation of previously published sources and requires documentation in the context of the TxDOT research projects.

The data were derived from over 220 historical USGS data reports that occupy approximately 8 linear feet of bookshelf space. In total, the database contains more than 1,650 storms for 91 mostly small rural and urban watersheds in Texas. A small watershed is considered to have a drainage area of less than about 20 square miles. Each storm is

${ }^{1}$ U.S. Geological Survey, Austin, Texas

2 Texas Tech University, Lubbock, Texas

${ }^{3}$ University of Houston, Houston, Texas

${ }^{4}$ Lamar University, Beaumont, Texas 
recorded by rainfall and runoff for USGS streamflow-gaging stations in the watersheds. The locations of the stations are shown in figure 1. Detailed location maps of the respective study areas are available in each of the original reports, referenced in figures 14-29. Finally, a comprehensive citation list for the reports is provided in the "References" section of this report. The cited reports are organized by database module and are listed in chronological order.

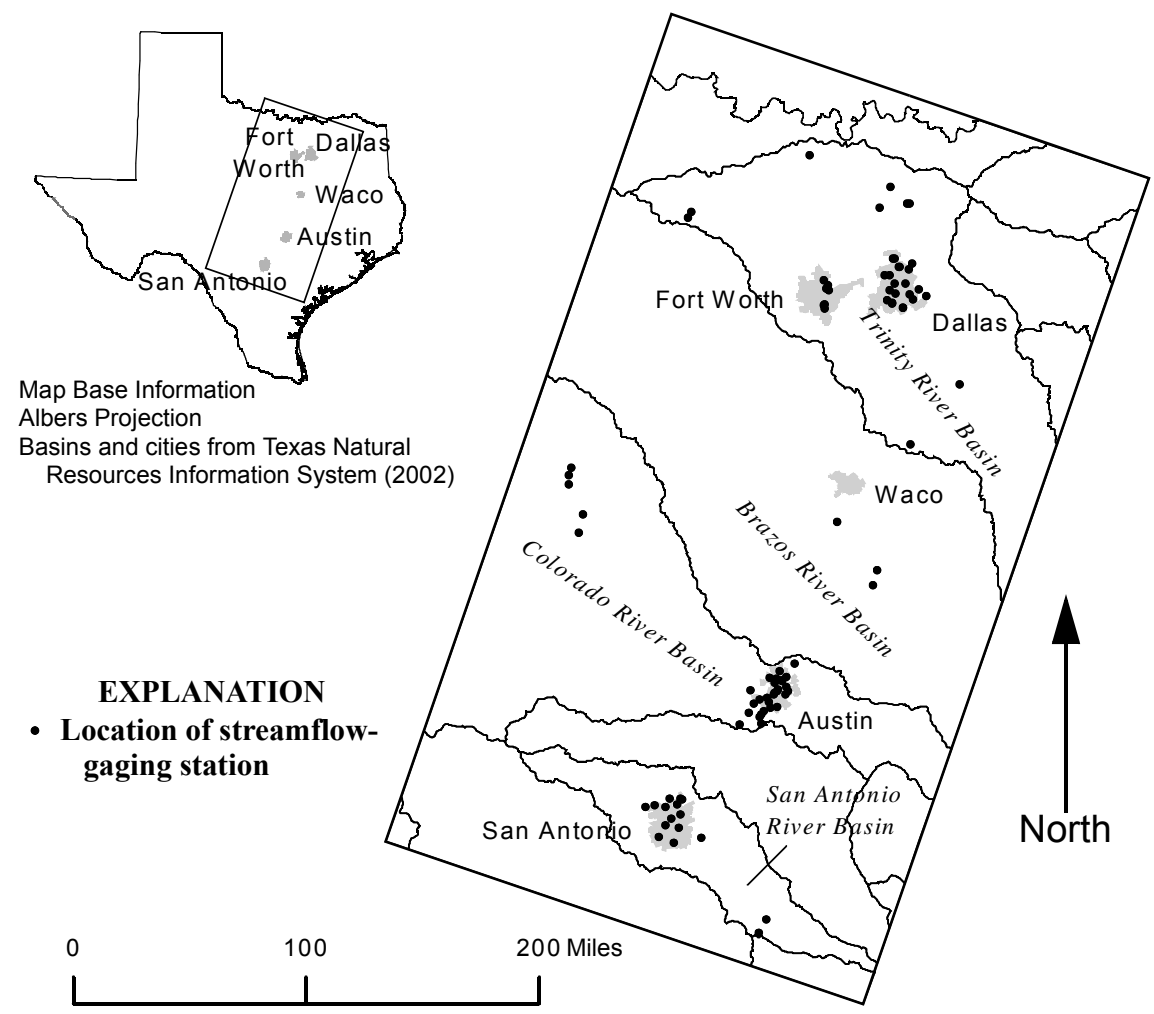

Figure 1. Locations of USGS streamflow-gaging stations represented in the database.

The purpose of this report is to document the database used by the investigators as part of the TxDOT research projects. The documentation of the database includes: (1) a basic description, (2) a listing of the original data sources, (3) the methods for converting data from original sources to the digital form used for the projects, (4) quality-assurance and quality-control measures implemented by the investigators, (5) description of limitations and caveats of the data, and (6) a complete listing of the data at the request of TxDOT.

\section{RAINFALL-RUNOFF DATABASE}

\section{Overview}

An overview of the database and its structure is described before details of the data are provided. The database contains rainfall and runoff values for watersheds in Texas. The database is separated into five "modules": austin, fortworth, da11as, sanantonio, and sma11rura1sheds. These modules correspond to a natural cataloging of the data reports. 
The austin, dallas, fortworth, and sanantonio modules contain rainfall-runoff data for watersheds within or near the cities of Austin, Dallas, Fort Worth, and San Antonio, Texas, respectively. The data collection activities in these cities are known as the USGS "urban studies." [The USGS also has conducted investigations and published data reports for the Houston area. These data were not used for the TxDOT research projects because of time and funding constraints and in part because of the contemporary publication of Liscum (2001).] The drainage network for each watershed generally comprises first to third order tributaries (see detailed location maps in "References" section). The land use included in the urban studies ranges from fully developed urban to natural or rural watersheds.

The sma11 rura1sheds module contains rainfall-runoff data for 12 intensively studied watershed units in rural regions of central to north-central Texas. The drainage network for each watershed generally comprises first to third order tributaries. The 12 watershed study units were studied in part by the historical development of the approximately 3,500 U.S. Soil Conservation Service (SCS) flood and soil-erosion control structures built in Texas between the period beginning in 1951-62 and ending in 1970-79. The USGS and SCS cooperatively collected data on a small fraction of these control structures and proximal watersheds.

The sma11ruralsheds module is further divided into four components: watersheds within the Brazos, Colorado, San Antonio, and Trinity River Basins. The Brazos River section contains four watershed study units: Cow Bayou, Green Creek, and Little Pond and North Elm Creeks watersheds. (Little Pond and North Elm Creeks neighbor one another, and data for these creeks were published together.) The Colorado River section contains two watershed study units: Deep Creek and Mukewater Creek. The San Antonio River section contains two watershed study units: Calaveras Creek and Escondido Creek. Finally, the Trinity River section contains five watershed study units: Elm Fork Trinity River, Honey Creek, Little Elm Creek, North Creek, and Pin Oak Creek.

The rainfall and runoff components of the database are described in separate sections of this report. Each "storm event" is recorded in the database by two data files. One file for the rainfall of the storm, and a second data file for the storm runoff. The storm events were chosen by the previous USGS analysts; the events are not inclusive of all rainfall-runoff events for the watersheds. Factors influencing whether an event was published in the original data reports include: instrument operation (data integrity), significance or magnitude of the storm, time of year, or simply the desire to have the data for a few storms per year published. The stations, for which incremental values of concomitant streamflow and rainfall for storm events are available, are listed in tables 1-5. 
Table 1. U.S. Geological Survey streamflow-gaging stations within the austin database module. $\left[\mathrm{mi}^{2}\right.$, square miles; Approx., approximate; SH, State Highway; U.S., U.S. Highway; FM, Farm to Market Road; IH, Interstate Highway. $1 \mathrm{mi}^{2}$ equals 2.59 square kilometers $\left(\mathrm{km}^{2}\right)$ ]

\begin{tabular}{|c|c|c|c|c|c|}
\hline $\begin{array}{c}\text { Station } \\
\text { no. }\end{array}$ & Station name & Latitude & Longitude & $\begin{array}{c}\text { Drainage } \\
\text { area } \\
\left(\mathrm{mi}^{2}\right)\end{array}$ & $\begin{array}{l}\text { Approx. } \\
\text { period of } \\
\text { record }\end{array}$ \\
\hline 08154700 & Bull Creek at Loop 360, Austin, Texas & $30^{\circ} 22^{\prime} 19^{\prime \prime}$ & $97^{\circ} 47^{\prime} 04^{\prime \prime}$ & 22.3 & $1978-86$ \\
\hline 08155200 & Barton Creek at SH 71, Oak Hill, Texas & $30^{\circ} 17^{\prime} 46^{\prime \prime}$ & $97^{\circ} 55^{\prime} 31^{\prime \prime}$ & 89.7 & $1978-82$ \\
\hline 08155300 & Barton Creek at Loop 360, Austin, Texas & $30^{\circ} 14^{\prime} 40^{\prime \prime}$ & $97^{\circ} 48^{\prime} 07^{\prime \prime}$ & 116.0 & $1979-86$ \\
\hline 08155550 & West Bouldin Creek at Riverside Drive, Austin, Texas & $30^{\circ} 15^{\prime} 49^{\prime \prime}$ & $97^{\circ} 45^{\prime} 17^{\prime \prime}$ & 3.12 & $1977-85$ \\
\hline 08156650 & Shoal Creek at Steck Avenue, Austin, Texas & $30^{\circ} 21^{\prime} 55^{\prime \prime}$ & $97^{\circ} 44^{\prime} 11^{\prime \prime}$ & 2.79 & $1975-82$ \\
\hline 08156700 & Shoal Creek at Northwest Park, Austin, Texas & $30^{\circ} 20^{\prime} 50^{\prime \prime}$ & $97^{\circ} 44^{\prime} 41^{\prime \prime}$ & 7.03 & $1976-83$ \\
\hline 08156750 & Shoal Creek at White Rock Drive, Austin, Texas & $30^{\circ} 20^{\prime} 21^{\prime \prime}$ & $97^{\circ} 44^{\prime} 50^{\prime \prime}$ & 7.56 & $1976-80$ \\
\hline 08156800 & Shoal Creek at 12th Street, Austin, Texas & $30^{\circ} 16^{\prime} 35^{\prime \prime}$ & $97^{\circ} 45^{\prime} 00^{\prime \prime}$ & 12.3 & $1975-86$ \\
\hline 08157000 & Waller Creek at 38th Street, Austin, Texas & $30^{\circ} 17^{\prime} 49^{\prime \prime}$ & $97^{\circ} 43^{\prime} 36^{\prime \prime}$ & 2.31 & $1967-80$ \\
\hline 08157500 & Waller Creek at 23rd Street, Austin, Texas & $30^{\circ} 17^{\prime} 08^{\prime \prime}$ & $97^{\circ} 44^{\prime} 01^{\prime \prime}$ & 4.13 & $1967-80$ \\
\hline 08158050 & Boggy Creek at U.S. 183, Austin, Texas & $30^{\circ} 15^{\prime} 47^{\prime \prime}$ & $97^{\circ} 40^{\prime} 20^{\prime \prime}$ & 13.1 & $1976-85$ \\
\hline 08158100 & Walnut Creek at FM 1325, Austin, Texas & $30^{\circ} 24^{\prime} 35^{\prime \prime}$ & $97^{\circ} 42^{\prime} 41^{\prime \prime}$ & 12.6 & $1976-86$ \\
\hline 08158200 & Walnut Creek at Dessau Road, Austin, Texas & $30^{\circ} 22^{\prime} 30^{\prime \prime}$ & $97^{\circ} 39^{\prime} 37^{\prime \prime}$ & 26.2 & $1976-86$ \\
\hline 08158380 & Little Walnut Creek at Georgian Drive Austin, Texas & $30^{\circ} 21^{\prime} 15^{\prime \prime}$ & $97^{\circ} 41^{\prime} 52^{\prime \prime}$ & 5.22 & $1985-86$ \\
\hline 08158400 & Little Walnut Creek at IH 35, Austin, Texas & $30^{\circ} 20^{\prime} 57^{\prime \prime}$ & $97^{\circ} 41^{\prime} 34^{\prime \prime}$ & 5.57 & $1976-81$ \\
\hline 08158500 & Little Walnut Creek at Manor Road, Austin, Texas & $30^{\circ} 18^{\prime} 34^{\prime \prime}$ & $97^{\circ} 40^{\prime} 04^{\prime \prime}$ & 12.1 & $1976-81$ \\
\hline 08158600 & Walnut Creek at Webberville Road, Austin, Texas & $30^{\circ} 16^{\prime} 59^{\prime \prime}$ & $97^{\circ} 39^{\prime} 17^{\prime \prime}$ & 51.3 & $1974-86$ \\
\hline 08158700 & Onion Creek near Driftwood, Texas & $30^{\circ} 04^{\prime} 59^{\prime \prime}$ & $98^{\circ} 00^{\prime} 29^{\prime \prime}$ & 124 & $1980-87$ \\
\hline 08158800 & Onion Creek at Buda, Texas & $30^{\circ} 05^{\prime} 09^{\prime \prime}$ & $97^{\circ} 50^{\prime} 52^{\prime \prime}$ & 166 & $1980-83$ \\
\hline 08158810 & Bear Creek below FM 1826, Driftwood, Texas & $30^{\circ} 09^{\prime} 19^{\prime \prime}$ & $97^{\circ} 56^{\prime} 23 ”$ & 12.2 & $1980-86$ \\
\hline 08158820 & Bear Creek at FM 1626, Manchaca, Texas & $30^{\circ} 08^{\prime} 25^{\prime \prime}$ & $97^{\circ} 50^{\prime} 50^{\prime \prime}$ & 24.0 & $1980-83$ \\
\hline 08158825 & Little Bear Creek at FM 1626, Manchaca, Texas & $30^{\circ} 07^{\prime} 31^{\prime \prime}$ & $97^{\circ} 51^{\prime} 43 ”$ & 21.0 & $1980-83$ \\
\hline 08158840 & Slaughter Creek at FM 1826, Austin, Texas & $30^{\circ} 12^{\prime} 32^{\prime \prime}$ & $97^{\circ} 54^{\prime} 11^{\prime \prime}$ & 8.24 & $1979-86$ \\
\hline 08158860 & Slaughter Creek at FM 2304, Austin, Texas & $30^{\circ} 09^{\prime} 43^{\prime \prime}$ & $97^{\circ} 49^{\prime} 55^{\prime \prime}$ & 23.1 & $1981-82$ \\
\hline 08158880 & Boggy Creek (south) at Circle "S" Road, Austin, Texas & $30^{\circ} 10^{\prime} 50^{\prime \prime}$ & $97^{\circ} 46^{\prime} 55^{\prime \prime}$ & 3.58 & $1976-86$ \\
\hline 08158920 & Williamson Creek at Oak Hill, Texas & $30^{\circ} 14^{\prime} 06^{\prime \prime}$ & $97^{\circ} 51^{\prime} 36^{\prime \prime}$ & 6.3 & $1979-84$ \\
\hline 08158930 & Williamson Creek at Manchaca Road, Austin, Texas & $30^{\circ} 13^{\prime} 16^{\prime \prime}$ & $97^{\circ} 47^{\prime} 36^{\prime \prime}$ & 19.0 & $1976-84$ \\
\hline 08158970 & Williamson Creek at Jimmy Clay Road, Austin, Texas & $30^{\circ} 11 ’ 21^{\prime \prime}$ & $97^{\circ} 43^{\prime} 56^{\prime \prime}$ & 27.6 & $1976-86$ \\
\hline 08159150 & Wilbarger Creek near Pflugerville, Texas & $30^{\circ} 27^{\prime} 16^{\prime \prime}$ & $97^{\circ} 36^{\prime} 02^{\prime \prime}$ & 4.61 & $1967-77$ \\
\hline
\end{tabular}


Table 2. U.S. Geological Survey streamflow-gaging stations within the da 11 as database module. $\left[\mathrm{mi}^{2}\right.$, square miles; Approx., approximate; U.S., U.S. Highway; IH, Interstate Highway; SH, State Highway. $1 \mathrm{mi}^{2}$ equals 2.59 square kilometers $\left(\mathrm{km}^{2}\right)$ ]

\begin{tabular}{|c|c|c|c|c|c|}
\hline $\begin{array}{c}\text { Station } \\
\text { no. }\end{array}$ & Station name & Latitude & Longitude & $\begin{array}{c}\text { Drainage } \\
\text { area } \\
\left(\mathrm{mi}^{2}\right)\end{array}$ & $\begin{array}{l}\text { Approx. } \\
\text { period of } \\
\text { record }\end{array}$ \\
\hline 08055580 & Joes Creek at Royal Lane, Dallas, Texas & $32^{\circ} 53^{\prime} 43^{\prime \prime}$ & $96^{\circ} 41^{\prime} 36^{\prime \prime}$ & 1.94 & $1974-79$ \\
\hline 08055600 & Joes Creek, Dallas, Texas & $32^{\circ} 51^{\prime} 41^{\prime \prime}$ & $96^{\circ} 52^{\prime} 27^{\prime \prime}$ & 7.51 & 1973-79 \\
\hline 08055700 & Bachman Branch, Dallas, Texas & $32^{\circ} 51^{\prime} 37^{\prime \prime}$ & $96^{\circ} 50^{\prime} 13^{\prime \prime}$ & 10.0 & 1964-79 \\
\hline 08056500 & Turtle Creek, Dallas, Texas & $32^{\circ} 48^{\prime} 26^{\prime \prime}$ & $96^{\circ} 48^{\prime} 08^{\prime \prime}$ & 7.98 & 1964-79 \\
\hline 08057020 & Coombs Creek at Sylvan Avenue, Dallas, Texas & $32^{\circ} 46^{\prime} 01^{\prime \prime}$ & $96^{\circ} 50^{\prime} 07^{\prime \prime}$ & 4.75 & $1965-79$ \\
\hline 08057050 & Cedar Creek at Bonnie View Road, Dallas, Texas & $32^{\circ} 44^{\prime} 50^{\prime \prime}$ & $96^{\circ} 47^{\prime} 44^{\prime \prime}$ & 9.42 & 1974-79 \\
\hline 08057120 & Spanky Branch at McCallum Lane, Dallas, Texas & $32^{\circ} 57^{\prime} 58^{\prime \prime}$ & $96^{\circ} 48^{\prime} 11^{\prime \prime}$ & 6.77 & $1973-78$ \\
\hline 08057130 & Rush Branch at Arapaho Road, Dallas, Texas & $32^{\circ} 57^{\prime} 45^{\prime \prime}$ & $96^{\circ} 47^{\prime} 44^{\prime \prime}$ & 1.22 & $1973-79$ \\
\hline 08057140 & Cottonwood Creek at Forest Lane, Dallas, Texas & $32^{\circ} 54^{\prime} 33^{\prime \prime}$ & $96^{\circ} 45^{\prime} 54^{\prime \prime}$ & 8.5 & $1973-78$ \\
\hline 08057160 & Floyd Branch at Forest Lane, Dallas, Texas & $32^{\circ} 54^{\prime} 33^{\prime \prime}$ & $96^{\circ} 45^{\prime} 34^{\prime \prime}$ & 4.17 & $1974-79$ \\
\hline 08057320 & Ash Creek at Highland Road, Dallas, Texas & $32^{\circ} 48^{\prime} 18^{\prime \prime}$ & $96^{\circ} 43^{\prime} 04^{\prime \prime}$ & 6.92 & $1973-78$ \\
\hline 08057415 & Elam Creek at Seco Boulevard, Dallas, Texas & $32^{\circ} 44^{\prime} 14^{\prime \prime}$ & $96^{\circ} 41^{\prime} 36^{\prime \prime}$ & 1.25 & $1973-79$ \\
\hline 08057418 & Fivemile Creek at Kiest Boulevard, Dallas, Texas & $32^{\circ} 42^{\prime} 19^{\prime \prime}$ & $96^{\circ} 51^{\prime} 32^{\prime \prime}$ & 7.65 & $1976-79$ \\
\hline 08057420 & Fivemile Creek at U.S. 77, Dallas, Texas & $32^{\circ} 41^{\prime} 15^{\prime \prime}$ & $96^{\circ} 49^{\prime} 22^{\prime \prime}$ & 13.2 & $1973-79$ \\
\hline 08057425 & Woody Branch at U.S. 77, Dallas, Texas & $32^{\circ} 40^{\prime} 58^{\prime \prime}$ & $96^{\circ} 49^{\prime} 22^{\prime \prime}$ & 11.5 & $1973-79$ \\
\hline 08057435 & Newton Creek at IH 635, Dallas, Texas & $32^{\circ} 39^{\prime} 19^{\prime \prime}$ & $96^{\circ} 44^{\prime} 41^{\prime \prime}$ & 5.91 & $1976-79$ \\
\hline 08057440 & Whites Branch at IH 635, Dallas Texas & $32^{\circ} 39^{\prime} 26^{\prime \prime}$ & $96^{\circ} 44^{\prime} 25^{\prime \prime}$ & 2.53 & $1976-79$ \\
\hline 08057445 & Prairie Creek at U.S. 175, Dallas, Texas & $32^{\circ} 42^{\prime} 17^{\prime \prime}$ & $96^{\circ} 40^{\prime} 11^{\prime \prime}$ & 9.03 & 1976-79 \\
\hline 08061620 & Duck Creek at Buckingham Road, Garland, Texas & $32^{\circ} 55^{\prime} 53^{\prime \prime}$ & $96^{\circ} 39^{\prime} 55^{\prime \prime}$ & 8.05 & 1973-79 \\
\hline 08061920 & South Mesquite Creek at SH 352, Mesquite, Texas & $32^{\circ} 46^{\prime} 09^{\prime \prime}$ & $96^{\circ} 37^{\prime} 18^{\prime \prime}$ & 13.4 & $1973-79$ \\
\hline 08061950 & South Mesquite Creek at Mercury Road, Mesquite, Texas & $32^{\circ} 43^{\prime} 32^{\prime \prime}$ & $96^{\circ} 34^{\prime} 12^{\prime \prime}$ & 23.0 & $1969-79$ \\
\hline
\end{tabular}

Table 3. U.S. Geological Survey streamflow-gaging stations within the fortworth database module. $\left[\mathrm{mi}^{2}\right.$, square miles; Approx., approximate; --, not available; IH, Interstate Highway; W, West. $1 \mathrm{mi}^{2}$ equals 2.59 square kilometers $\left.\left(\mathrm{km}^{2}\right)\right]$

\begin{tabular}{|c|c|c|c|c|c|}
\hline $\begin{array}{c}\text { Station } \\
\text { no. }\end{array}$ & Station name & Latitude & Longitude & $\begin{array}{c}\text { Drainage } \\
\text { area } \\
\left(\mathrm{mi}^{2}\right)\end{array}$ & $\begin{array}{l}\text { Approx. } \\
\text { period of } \\
\text { record }\end{array}$ \\
\hline-- & Seminary South Shopping Center, Fort Worth, Texas & -- & -- & 0.38 & $1970-76$ \\
\hline 08048520 & Sycamore Creek at IH 35-W, Fort Worth, Texas & $32^{\circ} 39^{\prime} 55^{\prime \prime}$ & $97^{\circ} 19^{\prime} 16^{\prime \prime}$ & 17.7 & $1970-77$ \\
\hline 08048530 & $\begin{array}{l}\text { Sycamore Creek tributary above Seminary South } \\
\text { Shopping Center, Fort Worth, Texas }\end{array}$ & $32^{\circ} 41^{\prime} 08^{\prime \prime}$ & $97^{\circ} 19^{\prime} 44^{\prime \prime}$ & 0.97 & $1970-77$ \\
\hline 08048540 & Sycamore Creek tributary at IH 35-W, Fort Worth, Texas & $32^{\circ} 41^{\prime} 18^{\prime \prime}$ & $97^{\circ} 19^{\prime} 11^{\prime}$ & 1.35 & $1970-76$ \\
\hline 08048550 & Dry Branch at Blandin Street, Fort Worth, Texas & $32^{\circ} 47^{\prime} 19^{\prime \prime}$ & $97^{\circ} 18^{\prime} 22^{\prime \prime}$ & 1.08 & 1969-76 \\
\hline 08048600 & Dry Branch at Fain Street, Fort Worth, Texas & $32^{\circ} 46^{\prime} 34^{\prime \prime}$ & $97^{\circ} 17^{\prime} 18^{\prime \prime}$ & 2.15 & 1969-77 \\
\hline 08048820 & Little Fossil Creek at IH 820, Fort Worth, Texas & $32^{\circ} 50^{\prime} 22^{\prime \prime}$ & $97^{\circ} 19^{\prime} 22^{\prime \prime}$ & 5.64 & 1969-77 \\
\hline 08048850 & Little Fossil Creek at Mesquite Street, Fort Worth, Texas & $32^{\circ} 48^{\prime} 33^{\prime \prime}$ & $97^{\circ} 17^{\prime} 28^{\prime \prime}$ & 12.3 & 1969-77 \\
\hline
\end{tabular}


Table 4. U.S. Geological Survey streamflow-gaging stations within the sanantonio database module. $\left[\mathrm{mi}^{2}\right.$, square miles; Approx., approximate; FM, Farm to Market Road. $1 \mathrm{mi}^{2}$ equals 2.59 square kilometers $\left.\left(\mathrm{km}^{2}\right)\right]$

\begin{tabular}{|c|c|c|c|c|c|}
\hline $\begin{array}{c}\text { Station } \\
\text { no. }\end{array}$ & Station name & Latitude & Longitude & $\begin{array}{c}\text { Drainage } \\
\text { area } \\
\left(\mathrm{mi}^{2}\right)\end{array}$ & $\begin{array}{l}\text { Approx. } \\
\text { period of } \\
\text { record }\end{array}$ \\
\hline 08177600 & Olmos Creek tributary at FM 1535, Shavano Park, Texas & $29^{\circ} 34^{\prime} 35^{\prime \prime}$ & $98^{\circ} 32^{\prime} 45^{\prime \prime}$ & 0.33 & $1970-81$ \\
\hline 08177700 & Olmos Creek at Dresden Drive, San Antonio, Texas & $29^{\circ} 29^{\prime} 56^{\prime \prime}$ & $98^{\circ} 30^{\prime} 36^{\prime \prime}$ & 21.2 & $1969-78$ \\
\hline 08178300 & Alazan Creek at St. Cloud Street, San Antonio, Texas & $29^{\circ} 27^{\prime} 29^{\prime \prime}$ & $98^{\circ} 32^{\prime} 59^{\prime \prime}$ & 3.26 & $1969-79$ \\
\hline 08178555 & $\begin{array}{l}\text { Harlendale Creek at West Harding Street, San Antonio, } \\
\text { Texas }\end{array}$ & $29^{\circ} 21^{\prime} 05^{\prime \prime}$ & $98^{\circ} 29^{\prime} 32^{\prime \prime}$ & 2.43 & $1977-80$ \\
\hline 08178600 & $\begin{array}{l}\text { Panther Springs Creek at FM } 2696 \text { near San Antonio, } \\
\text { Texas }\end{array}$ & $29^{\circ} 37^{\prime} 31^{\prime \prime}$ & $98^{\circ} 31^{\prime} 06^{\prime \prime}$ & 9.54 & $1969-75$ \\
\hline 08178620 & $\begin{array}{l}\text { Lorence Creek at Thousand Oaks Boulevard, San } \\
\text { Antonio, Texas }\end{array}$ & $29^{\circ} 35^{\prime} 24^{\prime \prime}$ & $98^{\circ} 27^{\prime} 47^{\prime \prime}$ & 4.05 & $1981-81$ \\
\hline 08178640 & West Elm Creek at San Antonio, Texas & $29^{\circ} 37^{\prime} 23^{\prime \prime}$ & $98^{\circ} 26^{\prime} 29^{\prime \prime}$ & 2.45 & $1976-79$ \\
\hline 08178645 & East Elm Creek at San Antonio, Texas & $29^{\circ} 37^{\prime} 04^{\prime \prime}$ & $98^{\circ} 25^{\prime} 41^{\prime \prime}$ & 2.33 & $1976-79$ \\
\hline 08178690 & $\begin{array}{l}\text { Salado Creek tributary at Bitters Road, San Antonio, } \\
\text { Texas }\end{array}$ & $29^{\circ} 31^{\prime} 36^{\prime \prime}$ & $98^{\circ} 26^{\prime} 25^{\prime \prime}$ & 0.26 & $1969-81$ \\
\hline 08178736 & Salado Creek tributary at Bee Street, San Antonio, Texas & $29^{\circ} 26^{\prime} 38^{\prime \prime}$ & $98^{\circ} 27^{\prime} 13^{\prime \prime}$ & 0.45 & $1972-76$ \\
\hline 08181000 & Leon Creek tributary at FM 1604, San Antonio, Texas & $29^{\circ} 35^{\prime} 14^{\prime \prime}$ & $98^{\circ} 37^{\prime} 40^{\prime \prime}$ & 5.57 & $1970-79$ \\
\hline 08181400 & Helotes Creek at Helotes, Texas & $29^{\circ} 34^{\prime} 42^{\prime}$ & $98^{\circ} 41^{\prime} 29^{\prime \prime}$ & 15.0 & $1969-81$ \\
\hline 08181450 & Leon Creek tributary at Kelly Air Force Base, Texas & $29^{\circ} 23^{\prime} 12^{\prime \prime}$ & $98^{\circ} 36^{\prime} 00^{\prime \prime}$ & 1.19 & 1969-79 \\
\hline
\end{tabular}

Description of the database layout is provided for clarity. A listing of the directory nomenclature of the data hierarchy for each module is shown in figures $2-6$. In each figure the "/" symbol is an individual directory separator. The parent module is shown at the beginning of each line, such as austin. For the four urban databases (figs. 2-5), the second entry in the line is the local watershed name, such as shoalcreek. For the sma11 ruralsheds module (fig. 6), the second entry in the line is a major river basin in Texas, such as BrazosBasin. The specific station is identified by sta\#\#\#\#\#\#\#_d, where \# represents a single digit in the USGS eight-digit station number. Following the station number component are two subdirectories: hyetos and hydros. These directories contain the actual data files. The hyetos directory contains the rainfall or hyetograph data files, and the hydros directory contains the runoff data files.

For brevity, a listing of the data file names are not provided in this section. However, the entire contents of the database are provided at the end of this report, and the data file names are shown in the header of the data files (see next two sections). The rainfall data files are named according to the following example: rain_sta08156800_1981_0523.dat. The station number follows the "sta" component, the next four digits represent the year (1981) and the following four digits represent the month (May) and day (23rd) of the storm. If multiple storms occurred on the same day for the same station, then another underscore and four digits representing the hour and minute of the start of the storm follows. Single digit values for month, day, hour, and minute are always prepended with a leading 0 ; this practice conveniently ensures that sorted lists of file names are in chronological order. 
Table 5. U.S. Geological Survey streamflow-gaging stations within the sma 11 rura 1 sheds database module.

$\left[\mathrm{mi}^{2}\right.$, square miles; Approx., approximate; Sub., Subwatershed; The * notes that two different drainage areas have been published for this station. $1 \mathrm{mi}^{2}$ equals 2.59 square kilometers $\left.\left(\mathrm{km}^{2}\right)\right]$

\begin{tabular}{|c|c|c|c|c|c|}
\hline $\begin{array}{c}\text { Station } \\
\text { no. }\end{array}$ & Station name & Latitude & Longitude & $\begin{array}{c}\text { Drainage } \\
\text { area } \\
\left(\mathrm{mi}^{2}\right)\end{array}$ & $\begin{array}{l}\text { Approx. } \\
\text { period of } \\
\text { record }\end{array}$ \\
\hline \multicolumn{6}{|c|}{ TRINITY RIVER BASIN } \\
\hline 08042650 & North Creek Sub. 28A near Jermyn, Texas & $33^{\circ} 14^{\prime} 52^{\prime \prime}$ & $98^{\circ} 19^{\prime} 19^{\prime \prime}$ & 6.82 & $1973-79$ \\
\hline 08042700 & North Creek near Jacksboro, Texas & $33^{\circ} 16^{\prime} 57^{\prime \prime}$ & $98^{\circ} 17^{\prime} 53^{\prime \prime}$ & 21.6 & 1959-79 \\
\hline 08050200 & Elm Fork Trinity River Sub. 6 near Muenster, Texas & $33^{\circ} 37^{\prime} 13^{\prime \prime}$ & $97^{\circ} 24^{\prime} 15^{\prime \prime}$ & .77 & $1961-70$ \\
\hline 08052630 & Little Elm Creek Sub. 10 near Gunter, Texas & $33^{\circ} 24^{\prime} 33^{\prime \prime}$ & $96^{\circ} 48^{\prime} 41^{\prime \prime}$ & 2.10 & $1966-76$ \\
\hline 08052700 & Little Elm Creek near Aubrey, Texas & $33^{\circ} 17^{\prime} 00^{\prime \prime}$ & $96^{\circ} 53 ’ 33^{\prime \prime}$ & 75.5 & $1959-76$ \\
\hline 08057500 & Honey Creek Sub. 11 near McKinney, Texas & $33^{\circ} 18^{\prime} 12^{\prime \prime}$ & $96^{\circ} 41^{\prime} 22^{\prime \prime}$ & 2.14 & $1960-70$ \\
\hline 08058000 & Honey Creek Sub.12 near McKinney, Texas & $33^{\circ} 18^{\prime} 20^{\prime \prime}$ & $96^{\circ} 40^{\prime} 12^{\prime \prime}$ & 1.26 & $1959-70$ \\
\hline 08063200 & Pin Oak Creek near Hubbard, Texas & $31^{\circ} 48^{\prime} 01^{\prime \prime}$ & $96^{\circ} 43^{\prime} 02^{\prime \prime}$ & 17.6 & $1959-71$ \\
\hline \multicolumn{6}{|c|}{ BRAZOS RIVER BASIN } \\
\hline 08094000 & Green Creek Sub. 1 near Dublin, Texas & $32^{\circ} 10^{\prime} 00^{\prime \prime}$ & $98^{\circ} 20^{\prime} 30^{\prime \prime}$ & $\begin{array}{c}* 3.18 / \\
4.19\end{array}$ & $1959-71$ \\
\hline 08096800 & Cow Bayou Sub. 4 near Bruceville, Texas & $31^{\circ} 19^{\prime} 59^{\prime \prime}$ & $97^{\circ} 16^{\prime} 02^{\prime \prime}$ & $\begin{array}{c}* 5.25 / \\
5.04\end{array}$ & $1959-75$ \\
\hline 08098300 & Little Pond Creek near Burlington, Texas & $31^{\circ} 01^{\prime} 35^{\prime \prime}$ & $96^{\circ} 59^{\prime} 17^{\prime \prime}$ & 22.2 & $1964-72$ \\
\hline 08108200 & North Elm Creek near Cameron, Texas & $30^{\circ} 55^{\prime} 52^{\prime \prime}$ & $97^{\circ} 01^{\prime} 13^{\prime \prime}$ & 48.6 & $1964-72$ \\
\hline \multicolumn{6}{|c|}{ COLORADO RIVER BASIN } \\
\hline 08136900 & Mukewater Creek Sub. 10A near Trickham, Texas & $31^{\circ} 39^{\prime} 01^{\prime \prime}$ & $99^{\circ} 13^{\prime} 30^{\prime \prime}$ & 21.8 & $1966-73$ \\
\hline 08137000 & Mukewater Creek Sub. 9 near Trickham, Texas & $31^{\circ} 41^{\prime} 40^{\prime \prime}$ & $99^{\circ} 12^{\prime} 18^{\prime \prime}$ & 4.02 & $1961-73$ \\
\hline 08137500 & Mukewater Creek at Trickham, Texas & $31^{\circ} 35^{\prime} 24^{\prime \prime}$ & $99^{\circ} 13^{\prime} 36^{\prime \prime}$ & 70.4 & $1959-61$ \\
\hline 08139000 & Deep Creek Sub. 3 near Placid,Texas & $31^{\circ} 17^{\prime} 25^{\prime \prime}$ & $99^{\circ} 09^{\prime} 22^{\prime \prime}$ & 3.42 & $1960-71$ \\
\hline 08140000 & Deep Creek Sub. 8 near Mercury, Texas & $31^{\circ} 24^{\prime} 08^{\prime \prime}$ & $99^{\circ} 07^{\prime} 17^{\prime \prime}$ & $\begin{array}{c}* 4.32 / \\
5.41\end{array}$ & $1960-71$ \\
\hline \multicolumn{6}{|c|}{ SAN ANTONIO RIVER BASIN } \\
\hline 08182400 & Calaveras Creek Sub. 6 near Elmendorf, Texas & $29^{\circ} 22^{\prime} 49^{\prime \prime}$ & $98^{\circ} 17^{\prime} 33^{\prime \prime}$ & 7.01 & $1961-71$ \\
\hline 08187000 & Escondido Creek Sub. 1 near Kenedy, Texas & $28^{\circ} 46^{\prime} 41^{\prime \prime}$ & $97^{\circ} 53^{\prime} 41^{\prime \prime}$ & 3.29 & $1959-71$ \\
\hline 08187900 & Escondido Creek Sub. 11 near Kenedy, Texas & $28^{\circ} 51^{\prime} 39^{\prime \prime}$ & $97^{\circ} 50^{\prime} 39^{\prime \prime}$ & 8.43 & $1962-70$ \\
\hline
\end{tabular}

The runoff data files are named similarly except unit instead of rain begins the file name: unit_sta08156800_1981_0523.dat. A special note is needed concerning the runoff data file name; the date-time values of the runoff data file name exactly match those in the rainfall file. The beginning of the storm rainfall dictated the date-time value in both rainfall and runoff file names. 
austin/BartonCreek/sta08155200_d/hydros austin/BartonCreek/sta08155200_d/hyetos austin/BartonCreek/sta08155300_d/hydros austin/BartonCreek/sta08155300_d/hyetos austin/BearCreek/sta08158810_d/hydros austin/BearCreek/sta08158810_d/hyetos austin/BearCreek/sta08158820_d/hydros austin/BearCreek/sta08158820_d/hyetos austin/BearCreek/sta08158825_d/hydros austin/BearCreek/sta08158825_d/hyetos austin/BoggyCreek/sta08158050_d/hydros austin/BoggyCreek/sta08158050_d/hyetos austin/BoggyCreekSouth/sta08158880_d/ hydros

austin/BoggyCreekSouth/sta08158880 d/ hyetos

austin/BullCreek/sta08154700_d/hydros austin/BullCreek/sta08154700_d/hyetos

austin/LittleWalnutCreek/sta08158380_d/ hydros

austin/LittlewalnutCreek/sta08158380_d/ hyetos

austin/OnionCreek/sta08158700_d/hydros austin/OnionCreek/sta08158700_d/hyetos austin/OnionCreek/sta08158800_d/hydros austin/Onioncreek/sta08158800_d/hyetos austin/ShoalCreek/sta08156650_d/hydros austin/ShoalCreek/sta08156650_d/hyetos austin/Shoalcreek/sta08156700_d/hydros austin/ShoalCreek/sta08156700_d/hyetos austin/ShoalCreek/sta08156750_d/hydros austin/ShoalCreek/sta08156750_d/hyetos austin/ShoalCreek/sta08156800_d/hydros austin/ShoalCreek/sta08156800_d/hyetos austin/SlaughterCreek/sta08158840_d/hydros
austin/SlaughterCreek/sta08158840_d/hyetos austin/SlaughterCreek/sta08158860_d/hydros austin/SlaughterCreek/sta08158860_d/hyetos austin/WallerCreek/sta08157000_d/hydros austin/WallerCreek/sta08157000_d/hyetos austin/WallerCreek/sta08157500_d/hydros austin/WallerCreek/sta08157500_d/hyetos austin/WalnutCreek/sta08158100_d/hydros austin/WalnutCreek/sta08158100_d/hyetos austin/WalnutCreek/sta08158200_d/hydros austin/WalnutCreek/sta08158200_d/hyetos austin/WalnutCreek/sta08158400_d/hydros austin/WalnutCreek/sta08158400_d/hyetos austin/WalnutCreek/sta08158500_d/hydros austin/WalnutCreek/sta08158500_d/hyetos austin/WalnutCreek/sta08158600_d/hydros austin/WalnutCreek/sta08158600_d/hyetos austin/WestBouldinCreek/sta08155550_d/ hydros

austin/WestBouldinCreek/sta08155550_d/ hyetos

austin/WilbargerCreek/sta08159150_d/hydros austin/WilbargerCreek/sta08159150_d/hyetos austin/WilliamsonCreek/sta08158920_d/ hydros

austin/WilliamsonCreek/sta08158920_d/ hyetos

austin/WilliamsonCreek/sta08158930_d/ hydros

austin/WilliamsonCreek/sta08158930_d/ hyetos

austin/WilliamsonCreek/sta08158970_d/ hydros

austin/WilliamsonCreek/sta08158970_d/ hyetos

Figure 2. Directory structure of the aust in database module. 


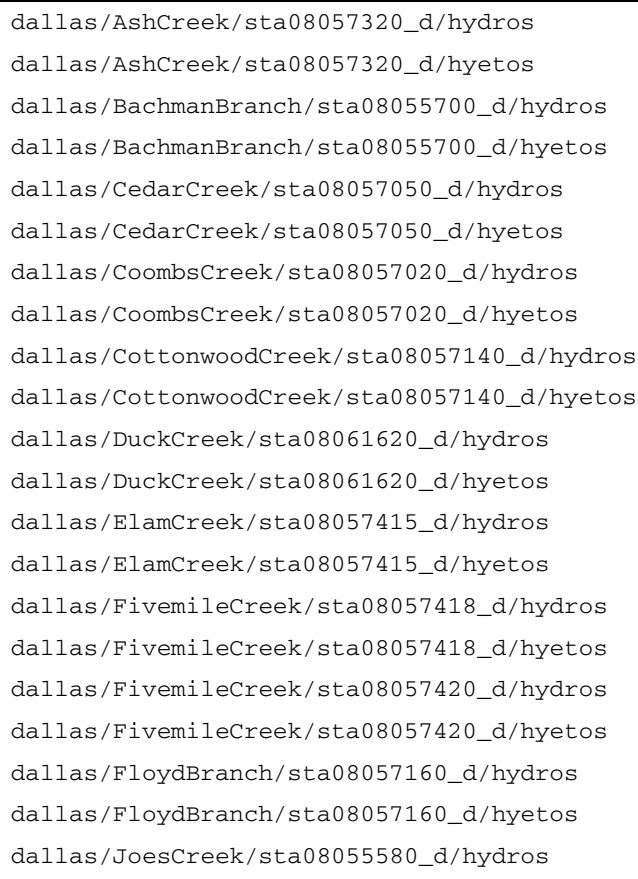

dallas/JoesCreek/sta08055580_d/hyetos dallas/JoesCreek/sta08055600_d/hydros dallas/JoesCreek/sta08055600_d/hyetos dallas/NewtonCreek/sta08057435_d/hydros dallas/NewtonCreek/sta08057435_d/hyetos dallas/PrairieCreek/sta08057445_d/hydros dallas/PrairieCreek/sta08057445_d/hyetos dallas/RushBranch/sta08057130_d/hydros dallas/RushBranch/sta08057130_d/hyetos dallas/SouthMesquite/sta08061920_d/hydros dallas/SouthMesquite/sta08061920_d/hyetos dallas/SouthMesquite/sta08061950_d/hydros dallas/SouthMesquite/sta08061950_d/hyetos dallas/SpankyCreek/sta08057120_d/hydros dallas/SpankyCreek/sta08057120_d/hyetos dallas/TurtleCreek/sta08056500_d/hydros dallas/TurtleCreek/sta08056500_d/hyetos dallas/WhitesBranch/sta08057440_d/hydros dallas/WhitesBranch/sta08057440_d/hyetos dallas/WoodyBranch/sta08057425_d/hydros dallas/WoodyBranch/sta08057425_d/hyetos

Figure 3. Directory structure of the da 11 as database module.

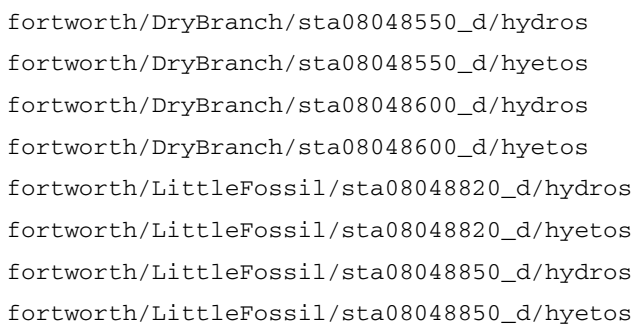

\section{Figure 4. Directory structure of the fortworth database module.}

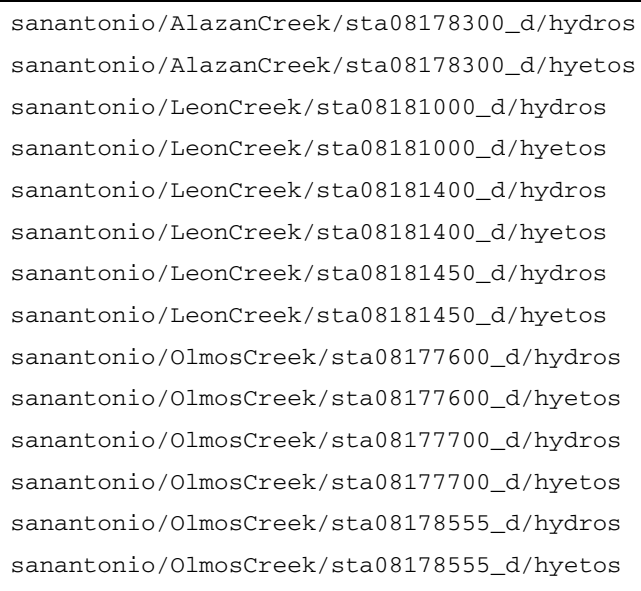

Figure 5. Directory structure of the sanantonio database module. 


\begin{tabular}{|c|c|}
\hline $\begin{array}{l}\text { smallruralsheds/BrazosBasin/CowBayou/ } \\
\text { sta08096800_d/hydros }\end{array}$ & $\begin{array}{l}\text { smallruralsheds/SanAntonioBasin/Escondido/ } \\
\text { sta08187000_d/hydros }\end{array}$ \\
\hline $\begin{array}{l}\text { smallruralsheds/BrazosBasin/CowBayou/ } \\
\text { sta08096800_d/hyetos }\end{array}$ & $\begin{array}{l}\text { smallruralsheds/SanAntonioBasin/Escondido/ } \\
\text { sta08187000_d/hyetos }\end{array}$ \\
\hline $\begin{array}{l}\text { smallruralsheds/BrazosBasin/Green/ } \\
\text { sta08094000_d/hydros }\end{array}$ & $\begin{array}{l}\text { smallruralsheds/SanAntonioBasin/Escondido/ } \\
\text { sta08187900_d/hydros }\end{array}$ \\
\hline $\begin{array}{l}\text { smallruralsheds/BrazosBasin/Green/ } \\
\text { sta08094000_d/hyetos }\end{array}$ & $\begin{array}{l}\text { smallruralsheds/SanAntonioBasin/Escondido/ } \\
\text { sta08187900_d/hyetos }\end{array}$ \\
\hline \multicolumn{2}{|l|}{$\begin{array}{l}\text { smallruralsheds/BrazosBasin/Pond-Elm/ } \\
\text { sta08098300 d/hydros }\end{array}$} \\
\hline $\begin{array}{l}\text { smallruralsheds/BrazosBasin/Pond-Elm/ } \\
\text { sta08098300_d/hyetos }\end{array}$ & sta08050200_d/hydros \\
\hline $\begin{array}{l}\text { smallruralsheds/BrazosBasin/Pond-Elm/ } \\
\text { sta08108200_d/hydros }\end{array}$ & $\begin{array}{l}\text { smallruralsheds/TrinityBasin/ElmFork/ } \\
\text { sta08050200_d/hyetos }\end{array}$ \\
\hline \multirow{2}{*}{$\begin{array}{l}\text { smallruralsheds/BrazosBasin/Pond-Elm/ } \\
\text { sta08108200_d/hyetos }\end{array}$} & $\begin{array}{l}\text { smallruralsheds/TrinityBasin/Honey/ } \\
\text { sta08057500_d/hydros }\end{array}$ \\
\hline & $\begin{array}{l}\text { smallruralsheds/TrinityBasin/Honey/ } \\
\text { sta08057500_d/hyetos }\end{array}$ \\
\hline $\begin{array}{l}\text { smallruralsheds/ColoradoBasin/Deep/ } \\
\text { sta08139000_d/hydros }\end{array}$ & $\begin{array}{l}\text { smallruralsheds/TrinityBasin/Honey/ } \\
\text { sta08058000_d/hydros }\end{array}$ \\
\hline $\begin{array}{l}\text { smallruralsheds/ColoradoBasin/Deep/ } \\
\text { sta08139000_d/hyetos }\end{array}$ & $\begin{array}{l}\text { smallruralsheds/TrinityBasin/Honey/ } \\
\text { sta08058000_d/hyetos }\end{array}$ \\
\hline $\begin{array}{l}\text { smallruralsheds/ColoradoBasin/Deep/ } \\
\text { sta08140000_d/hydros }\end{array}$ & $\begin{array}{l}\text { smallruralsheds/TrinityBasin/LittleElm/ } \\
\text { sta08052630_d/hydros }\end{array}$ \\
\hline $\begin{array}{l}\text { smallruralsheds/ColoradoBasin/Deep/ } \\
\text { sta08140000_d/hyetos }\end{array}$ & $\begin{array}{l}\text { smallruralsheds/TrinityBasin/LittleElm/ } \\
\text { sta08052630_d/hyetos }\end{array}$ \\
\hline $\begin{array}{l}\text { smallruralsheds/ColoradoBasin/Mukewater/ } \\
\text { sta08136900_d/hydros }\end{array}$ & $\begin{array}{l}\text { smallruralsheds/TrinityBasin/LittleElm/ } \\
\text { sta08052700_d/hydros }\end{array}$ \\
\hline $\begin{array}{l}\text { smallruralsheds/ColoradoBasin/Mukewater/ } \\
\text { sta08136900_d/hyetos }\end{array}$ & $\begin{array}{l}\text { smallruralsheds/TrinityBasin/LittleElm/ } \\
\text { sta08052700_d/hyetos }\end{array}$ \\
\hline $\begin{array}{l}\text { smallruralsheds/ColoradoBasin/Mukewater/ } \\
\text { sta08137000_d/hydros }\end{array}$ & $\begin{array}{l}\text { smallruralsheds/TrinityBasin/North/ } \\
\text { sta08042650_d/hydros }\end{array}$ \\
\hline $\begin{array}{l}\text { smallruralsheds/ColoradoBasin/Mukewater/ } \\
\text { sta08137000_d/hyetos }\end{array}$ & $\begin{array}{l}\text { smallruralsheds/TrinityBasin/North/ } \\
\text { sta08042650_d/hyetos }\end{array}$ \\
\hline $\begin{array}{l}\text { smallruralsheds/ColoradoBasin/Mukewater/ } \\
\text { sta08137500_d/hydros }\end{array}$ & $\begin{array}{l}\text { smallruralsheds/TrinityBasin/North/ } \\
\text { sta08042700_d/hydros }\end{array}$ \\
\hline $\begin{array}{l}\text { smallruralsheds/ColoradoBasin/Mukewater/ } \\
\text { sta08137500_d/hyetos }\end{array}$ & $\begin{array}{l}\text { smallruralsheds/TrinityBasin/North/ } \\
\text { sta08042700_d/hyetos }\end{array}$ \\
\hline smallruralsheds /SandantonioBasin/Calave & $\begin{array}{l}\text { smallruralsheds/TrinityBasin/Pinoak/ } \\
\text { sta08063200_d/hydros }\end{array}$ \\
\hline sta08182400_d/hydros & smallruralsheds/TrinityBasin/PinOak/ \\
\hline $\begin{array}{l}\text { smallruralsheds/SanAntonioBasin/Calaveras/ } \\
\text { sta08182400_d/hyetos }\end{array}$ & sta08063200_d/hyetos \\
\hline
\end{tabular}

Figure 6. Directory structure of the sma 17 rura 1 sheds database module.

\section{Rainfall Data-Hyetographs}

Each storm within the database is represented by a single rainfall data or hyetograph file. There are 1,657 hyetograph files in the database; these files contain approximately 57,900 lines of data. Three standard components of an hyetograph data file are provided in figure 7 . The three main components of the file are the header, the column titles, and the data records. The header of the file is identified by the lines containing the leading \# sign. A single field line containing the label or column titles follows the header. This line is delimited by one or more spaces. Finally, the data records, which also are one or more space delimited, make up the remainder of the file. A part of the data lines have been removed from the figure because of limited space. 


\begin{tabular}{|c|c|c|c|c|}
\hline \multicolumn{5}{|l|}{ \# HYETOGRAPH FILE } \\
\hline \multicolumn{5}{|c|}{ \# Filename=rain_sta08156800_1981_0523.dat } \\
\hline \\
\hline \\
\hline \\
\hline \multicolumn{5}{|c|}{ \# drainagearea $(\operatorname{mi2})=12.3$} \\
\hline \multicolumn{5}{|c|}{ \# DATE_TIME=date and time in MM/DD/YYYY@HH:MM } \\
\hline \multicolumn{5}{|c|}{ \# PRECIP1=1SHL raw recorded data } \\
\hline \multicolumn{5}{|c|}{ \# PRECIP2=2SHL raw recorded data } \\
\hline \multicolumn{5}{|c|}{ \# ACCUM_WTD_PRECIP=accumulated weighted precipitation in inches } \\
\hline \multicolumn{2}{|c|}{ DATE_TIME HOURS_PASSED PRECIP1 PRECIP2 ACCUM_WTD_PRECIP } & PRECIP1 & PRECIP2 & ACCUM_WTD_PRECIP \\
\hline 05/23/1981@00:00:00 & 0.0000 & 0.0000 & 0.0000 & 0.0000 \\
\hline 05/23/1981@23:30:00 & 23.5000 & 0.0700 & 0.0000 & 0.0200 \\
\hline 05/23/1981@23:45:00 & 23.7500 & 0.5700 & 0.1900 & 0.2800 \\
\hline 05/24/1981@00:00:00 & 24.0000 & 0.7400 & 0.5700 & 0.6100 \\
\hline 05/24/1981@00:30:00 & 24.5000 & 1.0400 & 0.9200 & 0.9500 \\
\hline 05/24/1981@01:00:00 & 25.0000 & 1.1300 & 1.0700 & 1.0800 \\
\hline \multicolumn{5}{|c|}{... portion cut for brevity } \\
\hline 05/25/1981@00:15:00 & 48.2500 & 8.5100 & 7.8800 & 8.0300 \\
\hline 05/25/1981@00:30:00 & 48.5000 & 5700 & 7.9800 & 8.1200 \\
\hline $05 / 25 / 1981 @ 00: 45: 00$ & 48.7500 & 8.6500 & 8.0200 & 8.1700 \\
\hline 05/25/1981@01:00:00 & 49.0000 & 8.7300 & 8.0700 & 8.2300 \\
\hline 05/25/1981@01:30:00 & 49.5000 & 8.7500 & 8.0800 & 8.2400 \\
\hline 05/25/1981@02:00:00 & 50.0000 & 8.7900 & 8.1400 & 8.3000 \\
\hline
\end{tabular}

Figure 7. Example hyetograph data file austin/shoalcreek/sta08156800_d/hyetos / rain_sta08156800_1981_0523.dat from the austin database module.

Some other aspects of the data file in figure 7 that need description are the values for PRECIP1, PRECIP2, and ACCUM_WTD_PRECIP. The ACCUM_WTD_PRECIP (accumulated weighted precipitation) is the best available estimate of rainfall for the entire watershed and was derived from the PRECIP1 and PRECIP2 values. For example, two rain gages, labeled PRECIP1 and PRECIP2, were operating during the May 1981 storm event in the Shoal Creek watershed. If additional rain gages were available during the event, then the labeling follows the PRECIP\# convention. Often, but not always, one of the rain gages was coincident with the streamflow-gaging station. The raw data are shown in the PRECIP\# fields. Sometimes the rainfall data collected for a watershed might include one or more weighing or volumetric rain gages, which previous authors determined more accurate data about total rainfall than the recording charts or tipping bucket rain gages. Volumetric rain gage data are not reported in the file, and hence are not explicitly represented in the database. The ACCUM_WTD_PRECIP values were computed by previous USGS analysts by amalgamated weighting of partial areas on the watershed and adjustments to the volumetric values when available. The partial area weight factors and volumetric adjustments are not reported for each rain gage for each storm in the database; the original data report is required to extract the appropriate weight and adjustment factors to convert the PRECIP\# data to the ACCUM_WTD_PRECIP data.

The 1,657 hyetographs for the five database modules are shown in figures $8-12$. In each of the five figures, the values composing each hyetograph are plotted against the concomitant time values in the top graphs (graphs A). The period of record available in each module is seen in graphs A. The separate watersheds within each database module are not differentiated. It is evident in graphs A that the number of storms represented per year is relatively uniform for the four urban database modules (figs. 8-11). Some minor clustering of events on a yearly basis is exhibited; this reflects concepts of "wet" and "dry" years. The sma11ruralsheds module (fig. 12) exhibits a marked reduction in the 
number of storm events per year starting about 1972 as the study units were decommissioned. The last storms available in the database occur in 1987 for the austin module. Although the USGS and numerous collaborating agencies continue to collect data in rural and urban areas in Texas, compilation and publication of both rainfall and runoff data for individual storms immediately applicable to the TxDOT research projects are not available after about 1987 (outside of the Houston area).

It is important to note that the total number of visually distinct hyetographs in graphs A does not equal the number of events seen in the other graphs (B and $\mathrm{C}$ ) or indicated in the figure title. This difference in count exists because numerous events typically occur simultaneously; a single storm system often affects many stations in the area surrounding and along the path of the system. This has the effect of causing many hyetographs to become masked in graphs A. Because many of the hyetographs occur on the same day or other intervals of time, the observed hyetographs are not each independent. Therefore, meteorological mechanisms generating each hyetograph are not random or statistically independent - the database does not contain as much independent information as suggested by the total number of hyetographs.

In order to visualize seasonal differences in the magnitude and the number of events, the year was converted to a common base (on a leap year) for the middle graphs (graphs B) in figures 8-12. A strong seasonal clustering of events is seen for the austin module (fig. 8). One cluster occurs between the middle of April and the middle of June, and a secondary cluster occurs in October. The da11as module (fig. 9) also exhibits a primary event cluster between the middle of April and the middle of June. Distinct clusters are harder to visualize in the fortworth module (fig. 10); although a weak cluster near the end of May might exist. The sanantonio module (fig. 11) exhibits a strong primary cluster between the middle of April and the middle of June. This is consistent with the austin module. A secondary cluster for the sanantonio module is less distinct and much longer lasting than that for the austin module. The cluster begins at the beginning of August and gradually tapers off towards the end of the year. The sma71ruralsheds module, which has the greatest range in geographic location of the five modules and includes areas proximate to the other four modules, exhibits two or three event clusters. One cluster occurs between the middle of April and the middle of June; a possible cluster near the end of July; and a final cluster between the end of August to the middle of October.

In the bottom graphs (graphs $\mathrm{C}$ ) in figures 8-12, each hyetograph is plotted against the HOURS_PASSED field. A substantial variation in the temporal distribution of relative rainfall intensity (slope or first derivative of the lines) exists. 

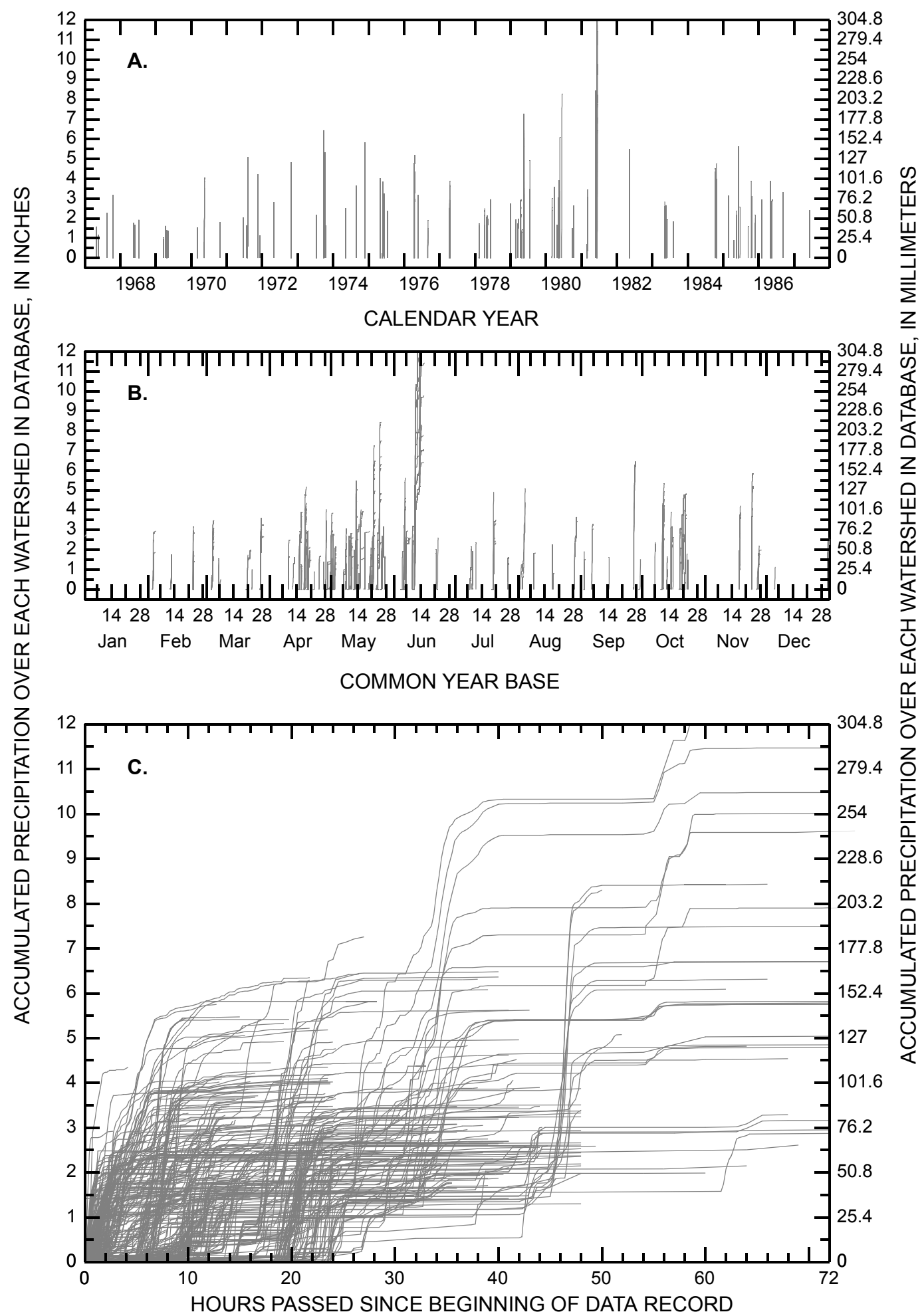

Figure 8. Hyetographs for austin database module—401 storm events represented. 


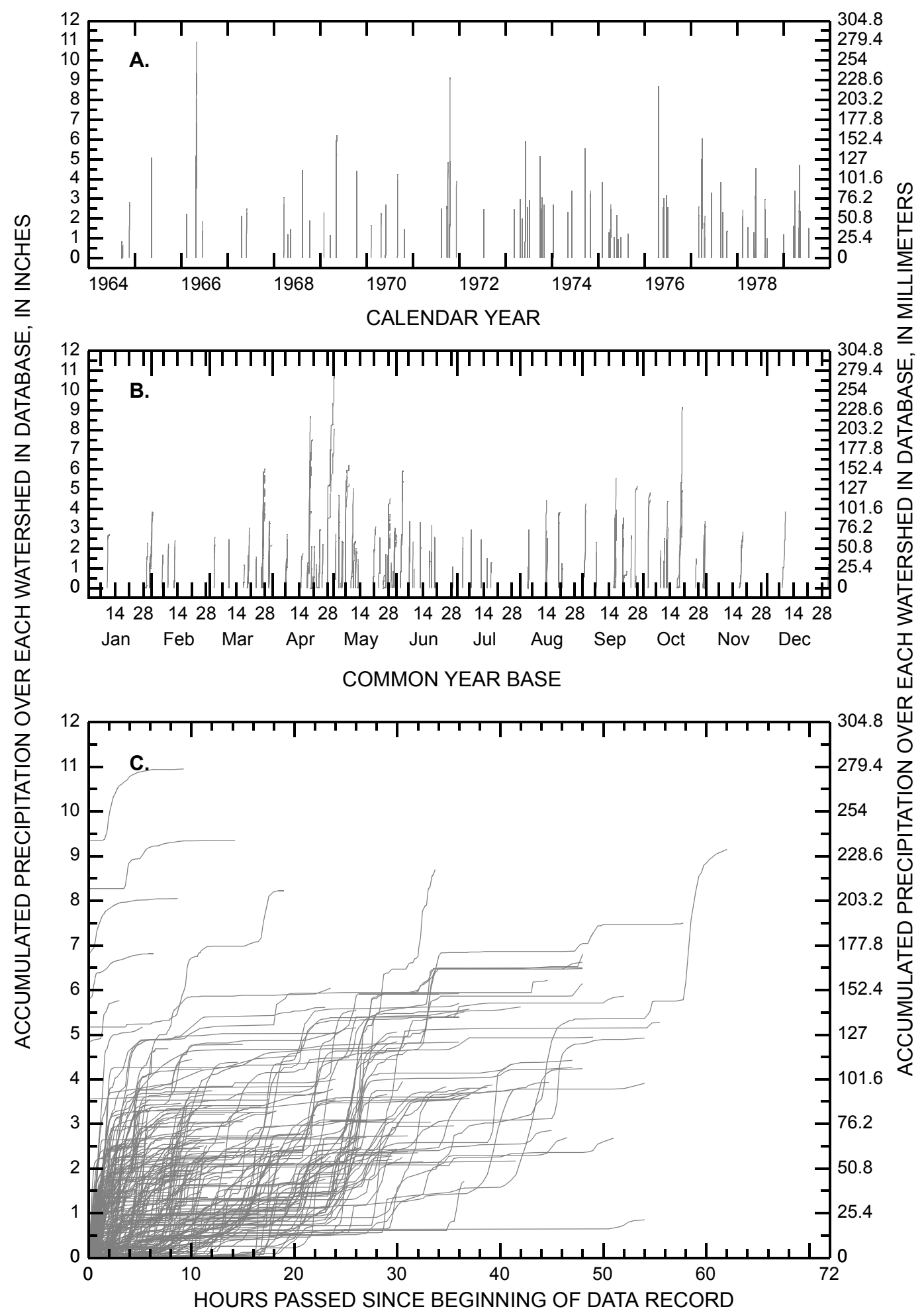

Figure 9. Hyetographs for da 11 as database module-240 storm events represented. 


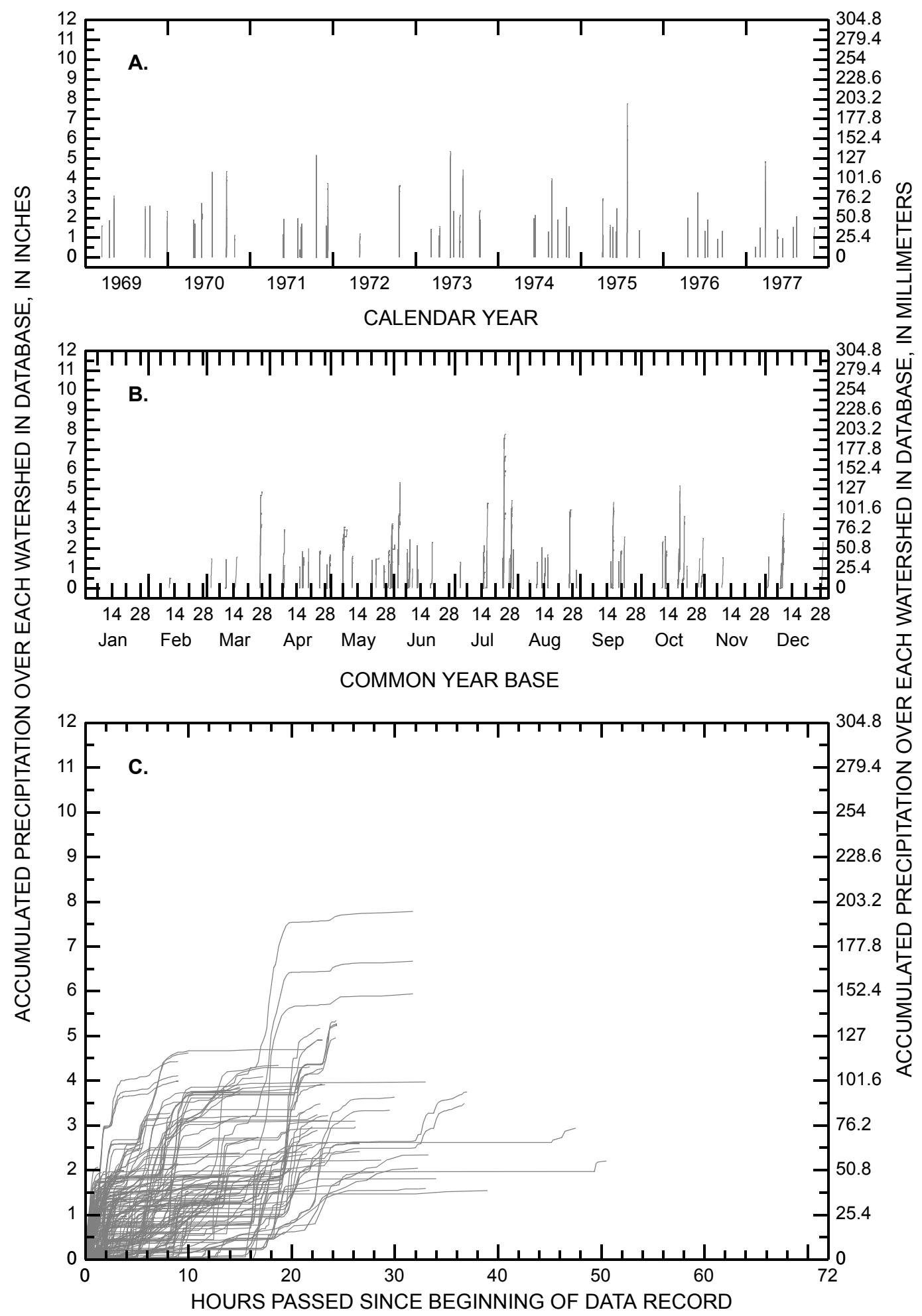

Figure 10. Hyetographs for fortworth database module—194 storm events represented. 


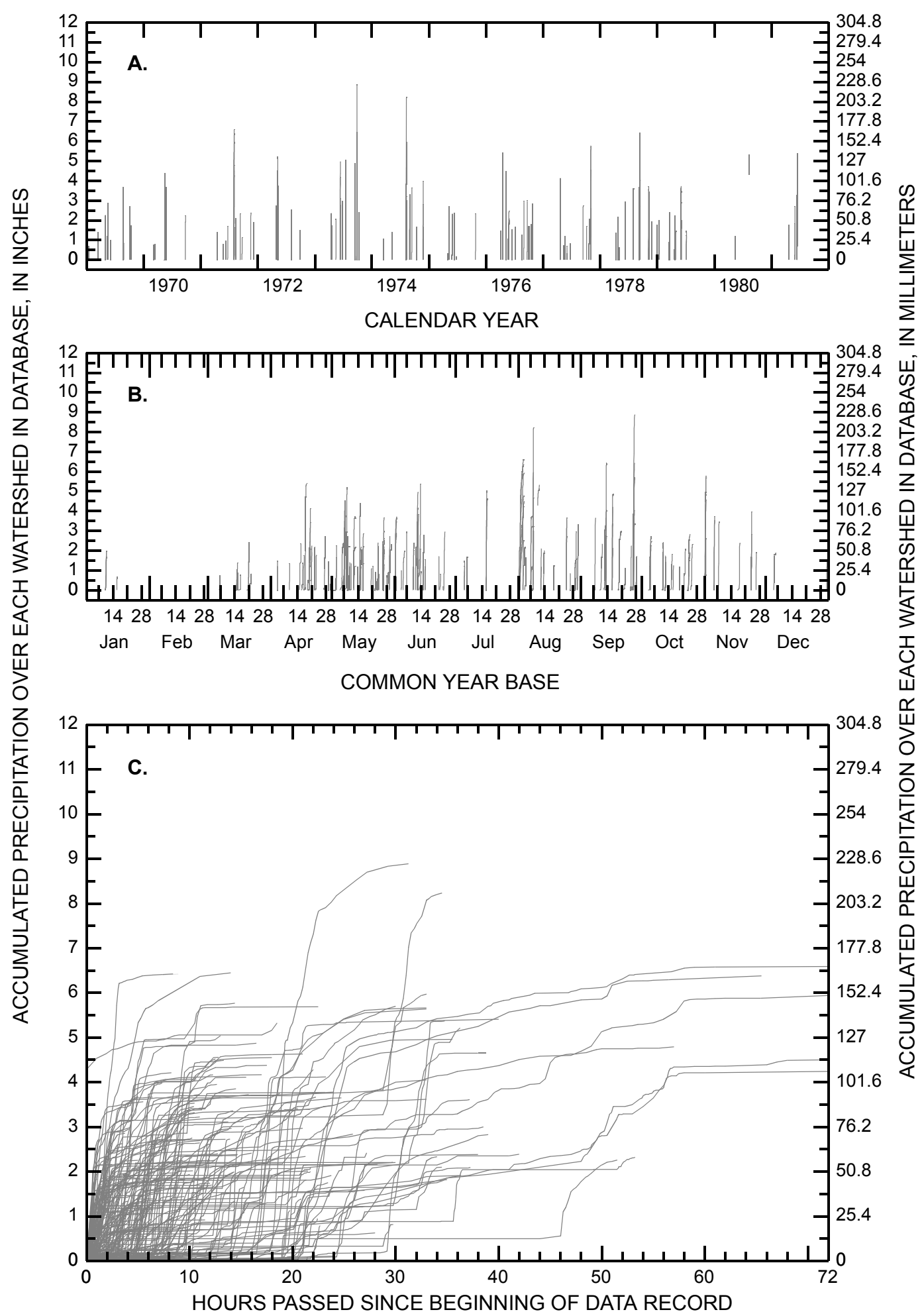

Figure 11. Hyetographs for sanantonio database module-215 storm events represented. 


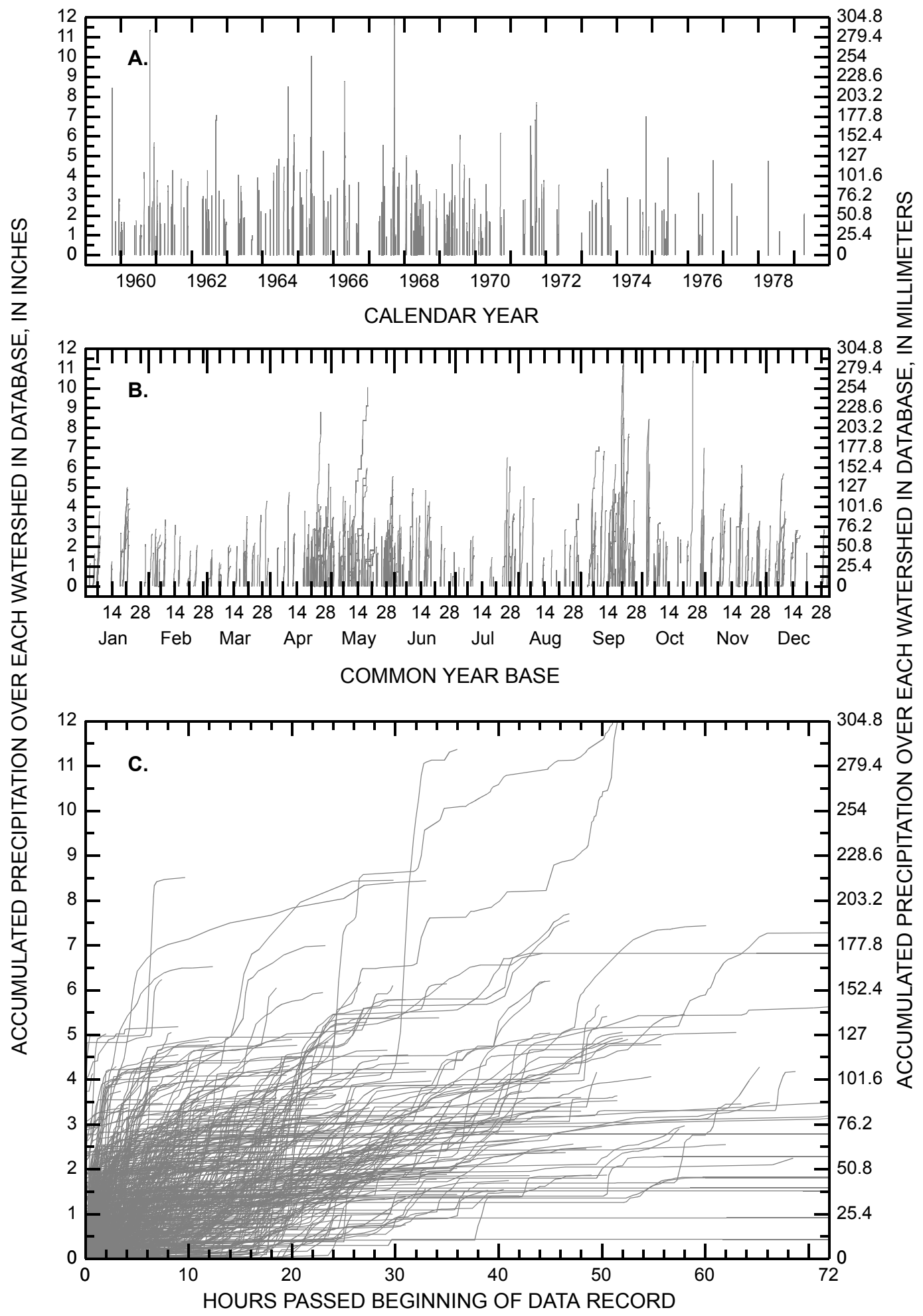

Figure 12. Hyetographs for sma 17 rura 1 sheds database module -607 storm events represented. 


\section{Runoff Data-Hydrographs}

The runoff for each storm within the database is represented by a single hydrograph file. There are 1,657 hydrograph files in the database; these files contain approximately 72,100 lines of data. Three standard components of a hydrograph file are provided in figure 13. The file format is closely related to the format for the hyetograph files. The three main components of the file are the header, the column titles, and the data records. The header of the file is identified by the lines containing the leading \# sign. A single field line containing the label or column titles follows the header. This line is delimited by one or more spaces. Finally, the data records, which also are space delimited, make up the remainder of the file. A part of the data lines have been removed from the figure because of limited space.

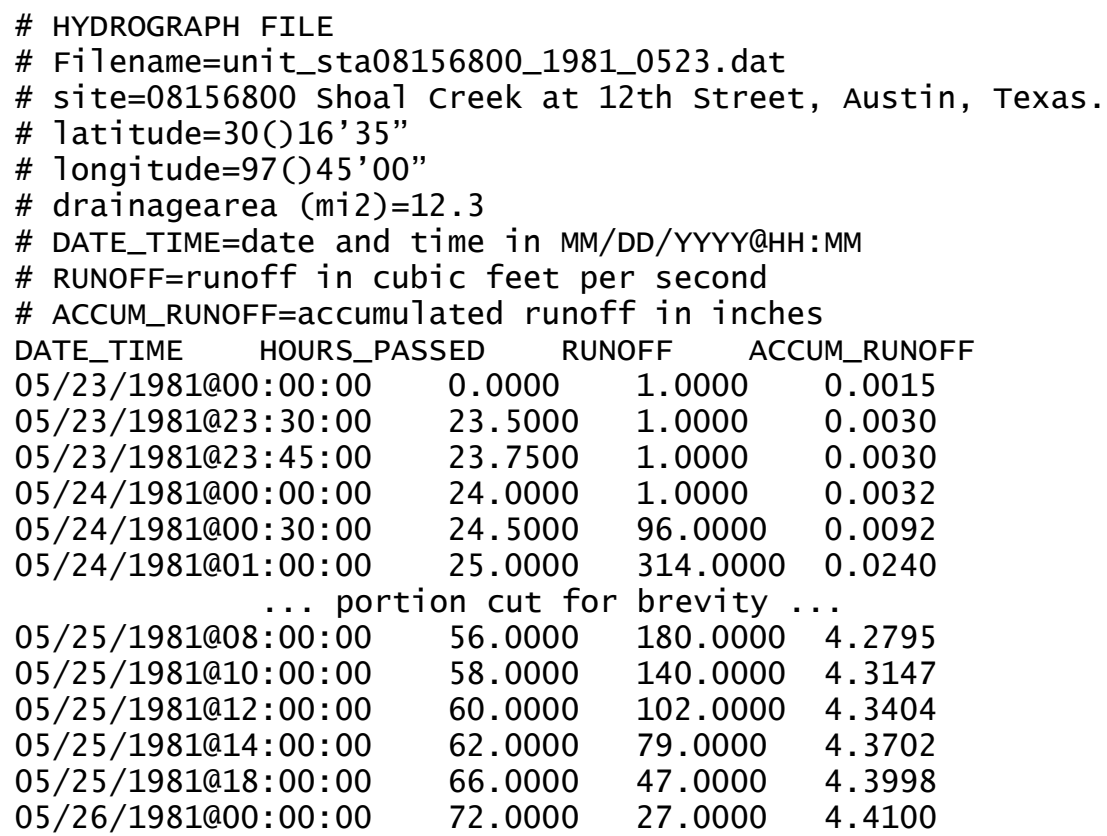

Figure 13. Example hydrograph data file austin/shoa1Creek/sta08156800_d/hyetos/ unit_sta08156800_1981_0523.dat from the austin database module.

Some other aspects of the data file in figure 13 that need description are the values for RUNOFF and ACCUM_RUNOFF. The RUNOFF is either inches of runoff between successive date-time stamps, or it is the instantaneous discharge in cubic feet per second for the corresponding date-time stamp. The USGS urban studies report RUNOFF in cubic feet per second; whereas, the reports represented in the sma11ruralsheds module have RUNOFF values reported in inches. The ACCUM_RUNOFF field contains the cumulative runoff in inches for the storm. An incremental runoff value in inches is computed from streamflow in cubic feet per second by

$$
R=2.58 \times 10^{-5} \times \frac{Q T}{A},
$$

where $R$ is runoff in inches, $Q$ is cubic feet per second, $T$ is time in minutes, and $A$ is drainage area in square miles. The $T$ value in eq. 1 is determined by the integration 
method; a mid-point method generally is used (see next section). A final note about the ACCUM_RUNOFF data is that base flow has not been removed (see "Cautions" section for further discussion).

\section{Note on Discharge}

An important component of the hydrograph data files is the backwards-computation of discharge (RUNOFF in the urban modules) in cubic feet per second from the ACCUM_RUNOFF in inches. All stations, except those with "subwatershed" in the station name, require that discharge is computed by a backwards-runoff centered-time finite difference (eq. 2).

The backwards-runoff centered-time finite difference is

$$
Q_{i}=645 \times A \times \frac{R_{i}-R_{i-1}}{0.5 \times\left(T_{i+1}-T_{i-1}\right)},
$$

where $Q_{i}$ is the discharge in cubic feet per second for the $i$ th HOURS_PASSED value, $R_{i}$ is the ACCUM_RUNOFF in inches for the $i$ th time, $T_{i}$ is the HOURS_PASSED value, and $A$ is the drainage area in square miles. The value 645 accounts for unit conversion.

For those stations that have "subwatershed" in the station name, a backwards-runoff backwards-time finite difference is used (eq. 3). An example subwatershed is station 08094000 Green Creek subwatershed no. 1 near Dublin, Texas (table 5). The reason for the different method is that the subwatersheds had hydrologic data collected on floodwater detention basins; inflow computations (the runoff) were made through change in storage conditions on the basin. Hence, runoff is in inches and not cubic feet per second. The peak discharge of inflow for the subwatersheds is computed by

$$
Q_{i}=645 \times A \times \frac{R_{i}-R_{i-1}}{T_{i}-T_{i-1}},
$$

where $Q_{i}$ is the discharge in cubic feet per second for the $i$ th HOURS_PASSED value, $R_{i}$ is the ACCUM_RUNOFF in inches for the $i$ th time, $T_{i}$ is the HOURS_PASSED value, and $A$ is the drainage area in square miles. The value 645 accounts for unit conversion.

\section{Quality Control and Quality Assurance}

The original data sources are in printed format and a range of tabulation styles: handwritten, dot matrix, hand-typed, and modern computer print. The research projects required digitally formatted data. The data were manually entered into an open-source computer text file versioning software system (http://www.cvshome.org) on a RedHat ${ }^{\mathrm{TM}}$ Linux $^{\mathrm{TM}}$ (http://www.redhat.com) server over the course of 2 years by approximately 20 individuals scattered between the four research entities. The data were derived from over 220 historical USGS data reports. The USGS provided database administrative support and off premises backup, and TTU administered the server in Lubbock. 
Absolute verification of the manually inputted data is not possible given the scale of the database and numerous individuals involved in data entry. However, during the course of database development, numerous custom Perl (http://www.perl.com and http:// www.perl.org) scripts were run to scan each line of data to search for anomalies and enhance data integrity. A summary of functions (not necessarily in order) performed by the scripts follows:

1. The scripts have confirmed that all date-time values are valid - that is the value is for a real date and time. The scripts are based on Perl date-time computation

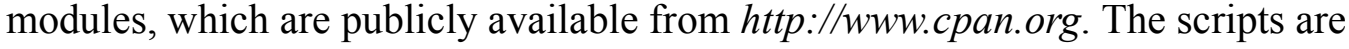
not able to test that the date-time value in the database is actually correct in respect to the original data.

2. The scripts have confirmed that the date-time values within each data file (rainfall and runoff) monotonically increase with time; a condition that is exhibited by the original data.

3. The scripts for each data file have cumulated the RUNOFF data and compared this total to the ACCUM_RUNOFF to identify anomalous data. A check based on explicit equality of the cumulated RUNOFF to the ACCUM_RUNOFF is not possible due to rounding and limited precision in the original data sources.

4. The scripts were used to compute the HOURS_PASSED field. The HOURS_PASSED field was not part of the original data sources.

5. The scripts have assured that the label line of the rainfall data files follow the DATE_TIME, HOURS_PASSED, PRECIP\#, and ACCUM_WTD_PRECIP convention.

6. The scripts have assured that the label line of the runoff data files follow the DATE_TIME, HOURS_PASSED, RUNOFF and ACCUM_RUNOFF convention.

7. The scripts have confirmed that cumulative values of rainfall and runoff are valid floating point numbers and that the numbers monotonically increase with time.

8. The data reports, particularly the urban study reports, consider the time value of zero-hundred hours (00:00) and the time value of twenty-four hundred hours (24:00) separately. Unfortunately 24:00 is not a recognized international standard in date-time computations. The time 24:00 is best considered as 00:00 of the next day. Scripts were necessary to remove 24:00 time stamps from the data files; careful manipulation of the rainfall and runoff values was required to maintain data integrity. However, the treatment of midnight varies from report to report, which influences the reliability of the ACCUM_RUNOFF values originally entered into the database. Interested readers can consult the data reports for further clarification. 
Furthermore, during the TxDOT research projects, the investigators each independently developed data-processing software to fulfill research objectives. These software programs frequently required digital comparison of the date-time values between the rainfall and runoff data files in the context of hydrologic research. Errors in the datetime stamps values were occasionally discovered. The data files then were compared to the original data sources and corrections applied. Others errors or inconsistencies were discovered in processing the data; when these errors were found, corrections were applied. Most error correction was completed by USGS staff in Austin.

A final script was run (May 2004) that recomputed the ACCUM_RUNOFF values using a mid-point integration method (half-width intervals were used for the initial and final RUNOFF values) from the RUNOFF values for the austin, dallas, fortworth, and sanantonio data modules. Such recomputation was deemed necessary to compensate for various original integration methods, data and intermediate computation precision (hand and early computer programs), various methods for the midnight transition, drainage area revisions (see "Cautions" section) and the fact that some original computations were hand made_-erroneous ACCUM_RUNOFF values often were detected.

\section{Cautions}

Several cautions regarding the database are listed in this section. These cautions influence how the data are processed and interpreted.

1. Drainage Area - The drainage areas for some of the stations particularly in the sma 17 rura1sheds module are known to have more than one published value. This influences the values for RUNOFF and sma 11 rura 1 sheds. These are identified in table 5. RUNOFF in the sma11 ruralsheds database is in inches. Inches of RUNOFF for a watershed is dependent on drainage area. The values in the sma 17 rura 1 sheds module are those available in the original data reports, and thus the drainage area used for the runoff calculations is the area available at the time that the original data were collected. The research team has explicitly chosen to leave the data in original values for the sma 11 rura1sheds module. However, the urban study modules have RUNOFF in cubic feet per second. Therefore, correction of the ACCUM_RUNOFF for revisions in drainage area was performed.

2. Latitude and Longitude - The latitude and longitude for some of the stations particularly in the sma11 rura1sheds module are known to have been revised (typos or errors in the reports) slightly over the course of the original data collection. The physical locations of the stations did not change. The research team believes that most accurate values are reflected in the tables. Exact location of the stations has little ramification on analysis objectives for the TxDOT research projects - unlike the changes in drainage area.

3. Rounding and Significant Figures - The data files were reformatted during quality-control and quality-assurance processing to assure file consistency. The reformatting included fixing decimal places to four. Four decimal places are necessary to maintain accuracy for the HOURS_PASSED values, which could have included data recorded as frequently as 5 minutes $(5 / 60=0.08 \overline{3})$. The RUNOFF values, when given in inches, were frequently recorded to but never greater than 
four decimal places. However, when RUNOFF values are in cubic feet per second, the original data were reported with various significant figures, but the values (discharge in cubic feet per second) are never more accurate than two decimal places. The ACCUM_RUNOFF values generally were reported to but never better than four decimal places. Users of the database should be aware that false precision is reflected in the data files. Users should also be aware that discharge computed using equations 2 or 3 in circumstances in which the difference between successive ACCUM_RUNOFF values is one or two significant figures (for example, $0.0001 \mathrm{inch}$ ) will only approximate the discharge reported in either the data files or the original reports.

4. Runoff as a Concept-The runoff data files in the database report total flow at the gaging station. Base flow was not removed from the data prior to recording in the original data reports, and hence, base flow is still a component of the data in this database. However, the majority of the stations represented are on small ephemeral streams; base flow represents a small component of the total flow at the station. The flow for the watershed frequently is zero at the beginning of the storms in the database.

5. Beginning and Ending of Runoff-The beginning of runoff data for a specific storm event report can be large relative to the peak discharge or total storm runoff. Likewise, the ending of the runoff data also can be large. Therefore uncertainty related to the actual beginning and ending of runoff exists for some storms.

6. Separate Files for Rainfall and Runoff - Several reasons exist for the use of two separate data files for each storm event in the database. The major reasons are:

a. The original data reports represented in the sma11 rura 1 sheds database module generally have separate tables for rainfall and runoff-logistically separate files made data entry easier.

b. The second reason is related to the first, which is that the original data reports represented in the sma11 rura1sheds database module do not necessarily have matching date-time stamps for rainfall and runoff. For example, during peak rainfall intensities the time interval could be a low as 5 minutes, while the runoff occurring at the same time might be in hourly intervals. If multiple datetime stamps were used, data file structure would be considerably more complicated and it would be more difficult to perform quality-control and quality-assurance tests. Sophisticated computer algorithms for date-time mathematics are required for some applications of concurrent processing of the rainfall and runoff data.

c. The beginning and ending times of rainfall hyetographs are not coincident with the beginning and ending times of a runoff hydrograph. Rainfall must occur before the runoff. Runoff hydrographs occur over a longer period of time relative to the rainfall; this is evidenced by the larger number of records of runoff data than rainfall data in the database.

d. TxDOT research project 0-4194 specifically involves the processing of rainfall data in pursuit of hyetograph definition in Texas. 


\section{SUMMARY}

The U.S. Geological Survey (USGS), Texas District, in collaborative peer-to-peer research projects with Lamar University, Texas Tech University, and the University of Houston, has developed a digital database of rainfall and runoff values for selected watersheds in Texas. The research was funded by the Texas Department of Transportation.

The data were derived from over 220 historical USGS data reports. The data were transcribed from hard-copy format to digital format over the course of 2 years. In total the database contains 1,657 storms for 91 rural and urban watersheds in Texas. Each storm is recorded by rainfall and runoff for USGS streamflow-gaging stations in the watersheds.

The composite database is divided into five database "modules." These modules are separated into four urban centers and are called austin, dallas, fortworth, and sanantonio, for the Austin, Dallas, Fort Worth, and San Antonio areas of Texas, respectively. The fifth module is referred to as the smal1ruralsheds database and contains clusters of intensively monitored small rural watershed study units within the Brazos River, Colorado River, San Antonio River, and Trinity River Basins of Texas.

Each storm event is recorded in the database by two data files. One file for the rainfall of the storm and a second data file for the storm runoff. Each storm within the database is represented by a single rainfall data or hyetograph file. There are 1,657 rainfall or hyetograph files in the database; these files contain approximately 57,900 lines of data. The hyetograph files contain date-time values and corresponding precipitation for one or more rain gages in the watershed; the files also contain the cumulative rainfall for the event. The runoff for each storm within the database is represented by a single hydrograph file. The hydrograph files contain date-time values, corresponding runoff (streamflow or change in reservoir storage), and cumulative runoff for the event. There are 1,657 hydrograph files in the database; these files contain approximately 72,100 lines of data.

An important component of the hydrograph data files is the discharge expressed in cubic feet per second for the event. The discharge is estimated from the cumulative runoff values. One of two types of finite-difference methods for discharge estimation are applicable for a given streamflow station. Each method is described, and a method to determine which discharge estimation type is applicable for a given watershed is described.

Discussion pertinent to the quality-control and quality-assurance method used on the database during the manual data-entry phase is provided. Finally, caveats, limitations, and miscellaneous discussion concerning the contents of the database are presented. 


\section{REFERENCES}

Liscum, Fred, 2001, Effects of urban development on stormwater runoff characteristics for the Houston, Texas, metropolitan area: U.S. Geological Survey Water-Resources Investigations Report 01-4071, 35 p.

\section{AUSTIN URBAN STUDIES}

U.S. Geological Survey, 1967, Basic data for urban hydrology study, Austin, Texas, 1967: U.S. Geological Survey, Texas District, 59 p.

U.S. Geological Survey, 1968, Compilation of hydrologic data, Austin, Texas, 1968: U.S. Geological Survey, Texas District, 68 p.

Robbins, W.D., 1969, Annual compilation and analysis of hydrologic data for urban studies in the Austin, Texas metropolitan area, 1969: U.S. Geological Survey, Texas District, $46 \mathrm{p}$.

VanZandt, J.K., 1972, Annual compilation and analysis of hydrologic data for urban studies in the Austin, Texas metropolitan area, 1970: U.S. Geological Survey OpenFile Report, 70 p.

Tovar, F.H., 1973, Annual compilation and analysis of hydrologic data for urban studies in the Austin, Texas metropolitan area, 1971: U.S. Geological Survey Open-File Report, $73 \mathrm{p}$.

Wehmeyer, E.E., 1974, Hydrologic data for urban studies in the Austin, Texas metropolitan area, 1972: U.S. Geological Survey Open-File Report, 49 p.

Mitchell, R.N., 1975, Hydrologic data for urban studies in the Austin, Texas metropolitan area, 1973: U.S. Geological Survey Open-File Report, 61 p.

Mitchell, R.N., 1976, Hydrologic data for urban studies in the Austin, Texas metropolitan area, 1974: U.S. Geological Survey Open-File Report, 60 p.

Choffel, K.L., Roddy, W.R., and Mitchell, R.N., 1977, Hydrologic data for urban studies in the Austin, Texas metropolitan area, 1975: U.S. Geological Survey Open-File Report 76-885, 126 p.

Maderak, M.L., Gordon, J.D., and Mitchell, R.N., 1978, Hydrologic data for urban studies in the Austin, Texas metropolitan area, 1976: U.S. Geological Survey Open-File Report 78-457, 263 p.

Slade, R.M., Jr., Gordon, J.D., and Mitchell, R.N., 1979, Hydrologic data for urban studies in the Austin, Texas metropolitan area, 1977: U.S. Geological Survey Open-File Report 79-271, 192 p.

Slade, R.M., Jr., Dorsey, M.E., Gordon, J.D., and Mitchell, R.N., 1980, Hydrologic data for urban studies in the Austin, Texas metropolitan area, 1978: U.S. Geological Survey Open-File Report 80-728, 227 p.

Slade, R.M., Jr., Dorsey, M.E., Gordon, J.D., Mitchell, R.N., and Gaylord, J.L., 1981, Hydrologic data for urban studies in the Austin, Texas metropolitan area, 1979: U.S. Geological Survey Open-File Report 81-628, 281 p. 
Slade, R.M., Jr., Gaylord, J.L., Dorsey, M.E., Mitchell, R.N., and Gordon, J.D., 1982, Hydrologic data for urban studies in the Austin, Texas metropolitan area, 1980: U.S. Geological Survey Open-File Report 82-506, 264 p.

Massey, B.C., Reeves, W.E., and Lear, W.A., 1982, Flood of May 24-25, 1981, in the Austin, Texas metropolitan area: U.S. Geological Survey Hydrologic Investigations Atlas HA-656, 2 p.

Slade, R.M., Jr., Veenhuis, J.E., Dorsey, M.E., Gardiner, H., and Smith, A.E., 1983, Hydrologic data for urban studies in the Austin, Texas metropolitan area, 1981: U.S. Geological Survey Open-File Report 83-44, 293 p.

Slade, R.M., Jr., Veenhuis, J.E., Dorsey, M.E., Stewart, S.L., and Ruiz, L.M., 1984, Hydrologic data for urban studies in the Austin, Texas metropolitan area, 1982: U.S. Geological Survey Open-File Report 84-061, 196 p.

Gordon, J.D., Pate, D.L., and Dorsey, M.E., 1985, Hydrologic data for urban studies in the Austin, Texas metropolitan area, 1983: U.S. Geological Survey Open-File Report 85-172, $154 \mathrm{p}$.

Gordon, J.D., Pate, D.L., and Dorsey, M.E., 1986, Hydrologic data for urban studies in the Austin metropolitan area, Texas, 1984: U.S. Geological Survey Open-File Report 85-676, $92 \mathrm{p}$.

Veenhuis, J.E. and Gannett, D.G., 1986, The effects of urbanization on floods in the Austin metropolitan area, Texas: U.S. Geological Survey Water-Resources Investigations Report 86-4069, 65 p.

Gordon, J.D., Pate, D.L., and Dorsey, M.E., 1987, Hydrologic data for urban studies in the Austin metropolitan area, Texas, 1985: U.S. Geological Survey Open-File Report $87-224,170$ p.

Gordon, J.D., Pate, D.L., and Slagle, D.L., 1988, Hydrologic data for urban studies in the Austin metropolitan area, Texas, 1986: U.S. Geological Survey Open-File Report 87-768, $144 \mathrm{p}$. 


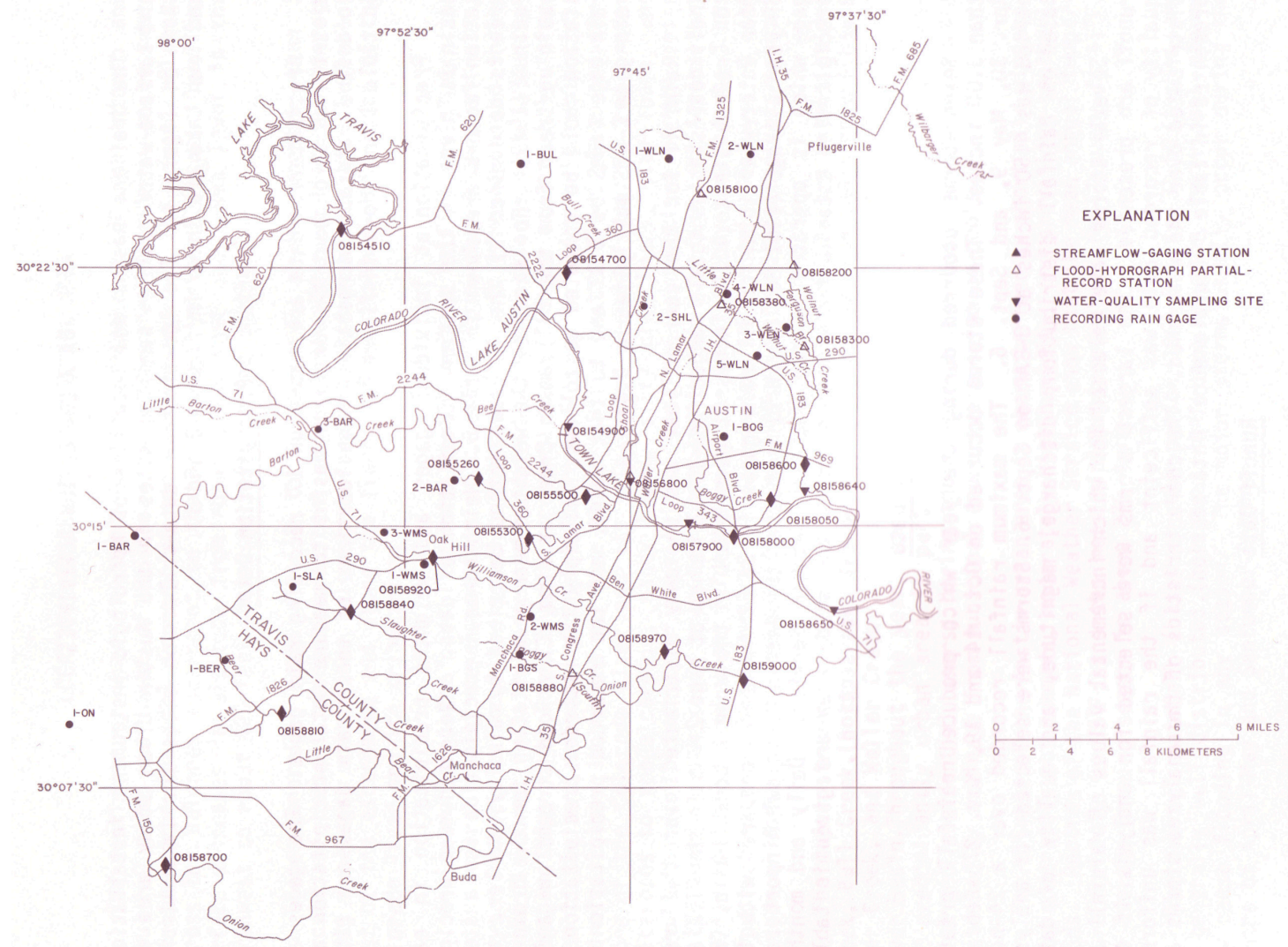

Figure 14. Location map for aust in database module adopted from Gordon, Pate, and Slagle, 1988.

\section{DALLAS URBAN STUDIES}

U.S. Geological Survey, 1965, Basic data for urban hydrology study, Dallas, Texas, 1965: U.S. Geological Survey, Texas District, 80 p.

U.S. Geological Survey, 1966, Basic data for urban hydrology study, Dallas, Texas, 1966:

U.S. Geological Survey, Texas District, 198 p.

U.S. Geological Survey, 1967, Basic data for urban hydrology study, Dallas, Texas, 1967: U.S. Geological Survey, Texas District, 80 p.

U.S. Geological Survey, 1968, Basic data for urban hydrology study, Dallas, Texas, 1968: U.S. Geological Survey, Texas District, 103 p.

Dempster, G.R., Jr. and Massey, B.C., 1971, Annual compilation and analysis of hydrologic data for urban studies in the Dallas, Texas metropolitan area, 1969: U.S. Geological Survey, Texas District, 136 p.

Dempster, G.R., Jr. and Massey, B.C., 1972, Annual compilation and analysis of hydrologic data for urban studies in the Dallas, Texas metropolitan area, 1970: U.S. Geological Survey, Texas District, 122 p. 
Massey, B.C., 1973, Annual compilation and analysis of hydrologic data for urban studies in the Dallas, Texas metropolitan area, 1971: U.S. Geological Survey, Texas District, $84 \mathrm{p}$.

Massey, B.C. and Wood, C.M., 1974, Hydrologic data for urban studies in the Dallas, Texas metropolitan area, 1972: U.S. Geological Survey Open-File Report, 136 p.

Dempster, G.R., Jr., 1974, Effects of urbanization on floods in the Dallas, Texas metropolitan area: U.S. Geological Survey Water-Resources Investigations Report $60-73,51 \mathrm{p}$.

Hampton, B.B., 1975, Hydrologic data for urban studies in the Dallas, Texas metropolitan area, 1973: U.S. Geological Survey Open-File Report, 146 p.

Hampton, B.B., 1976, Hydrologic data for urban studies in the Dallas, Texas metropolitan area, 1974: U.S. Geological Survey Open-File Report, 182 p.

Hampton, B.B. and Wood, C.M.,1977, Hydrologic data for urban studies in the Dallas, Texas metropolitan area, 1975: U.S. Geological Survey Open-File Report 77-381, 209 p.

Hampton, B.B. and Wood, C.M., 1978, Hydrologic data for urban studies in the Dallas, Texas metropolitan area, 1976: U.S. Geological Survey Open-File Report 78-437, $161 \mathrm{p}$.

Hampton, B.B. and Wood, C.M., 1979, Hydrologic data for urban studies in the Dallas, Texas metropolitan area, 1977: U.S. Geological Survey Open-File Report 79-562, $146 \mathrm{p}$.

Hampton, B.B. and Wood, C.M., 1980, Hydrologic data for urban studies in the Dallas, Texas metropolitan area, 1978: U.S. Geological Survey Open-File Report 80-1004, $114 \mathrm{p}$.

Wood, C.M., Butler, H.S., and Benton, J.D., 1981, Hydrologic data for urban studies in the Dallas, Texas metropolitan area, 1979: U.S. Geological Survey Open-File Report 81-491, $147 \mathrm{p}$.

Land, L.F., Schroeder, E.E., and Hampton, B.B., 1982, Techniques for estimating the magnitude and frequency of floods in the Dallas-Fort Worth metropolitan area, Texas: U.S. Geological Survey Water-Resources Investigations Report 82-18, 55 p. 


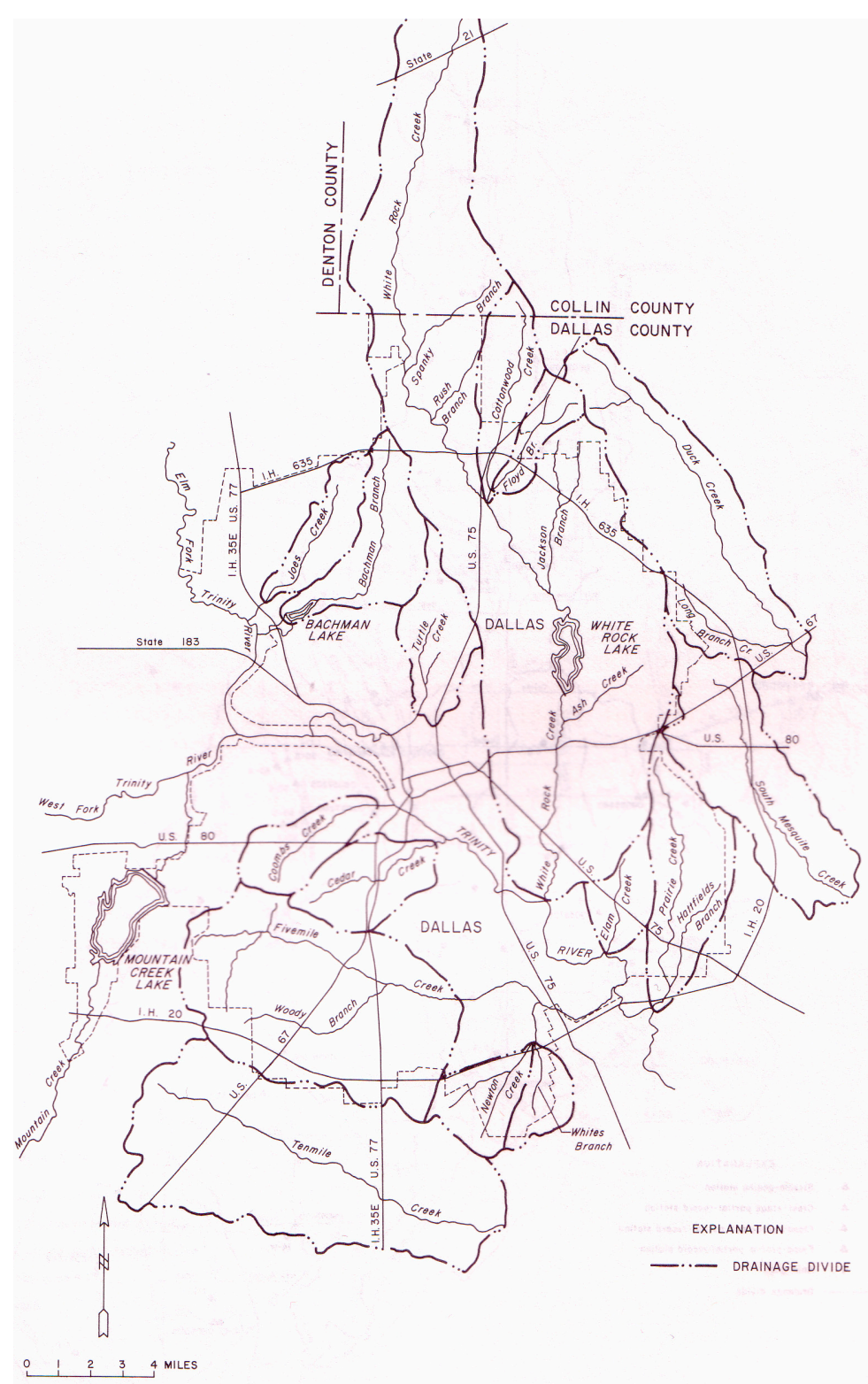

Figure 15. Location map for da 17 as database module adopted from Wood, Butler, and Benton, 1981.

\section{FORT WORTH URBAN STUDIES}

Dempster, G.R., Jr., and Massey, B.C., 1969, Annual compilation and analysis of hydrologic data for urban studies in the Fort Worth, Texas metropolitan area, 1969: U.S. Geological Survey Open-File Report, 50 p.

Dempster, G.R., Jr., and Massey, B.C., 1972, Annual compilation and analysis of hydrologic data for urban studies in the Fort Worth, Texas metropolitan area, 1970: U.S. Geological Survey Open-File Report, 89 p. 
Hampton, B.B., 1973, Annual compilation and analysis of hydrologic data for urban studies in the Fort Worth, Texas metropolitan area, 1971: U.S. Geological Survey Open-File Report, $77 \mathrm{p}$.

Hampton, B.B., 1974, Hydrologic data for urban studies in the Fort Worth, Texas metropolitan area, 1972: U.S. Geological Survey Open-File Report, 123 p.

Hampton, B.B., 1975, Hydrologic data for urban studies in the Fort Worth, Texas metropolitan area, 1973: U.S. Geological Survey Open-File Report, 129 p.

Slade, R.M., Jr., and Taylor, J.M., 1976, Hydrologic data for urban studies in the Fort Worth, Texas metropolitan area, 1974: U.S. Geological Survey Open-File Report, $100 \mathrm{p}$.

Slade, R.M., Jr., and Taylor, J.M., 1977, Hydrologic data for urban studies in the Fort Worth, Texas metropolitan area, 1975: U.S. Geological Survey Open-File Report $77-266,96 \mathrm{p}$.

Slade, R.M., Jr., and Taylor, J.M., 1978, Hydrologic data for urban studies in the Fort Worth, Texas metropolitan area, 1976: U.S. Geological Survey Open-File Report 77-770, $84 \mathrm{p}$.

Slade, R.M., Jr., Taylor, J.M., and Haynes, D.L., 1979, Hydrologic data for urban studies in the Fort Worth, Texas metropolitan area, 1977: U.S. Geological Survey Open-File Report 79-216, 39 p.

Land, L.F., Schroeder, E.E., and Hampton, B.B., 1982, Techniques for estimating the magnitude and frequency of floods in the Dallas-Fort Worth metropolitan area, Texas: U.S. Geological Survey Water-Resources Investigations Report 82-18, 55 p. [also listed in DALLAS URBAN STUDIES section] 


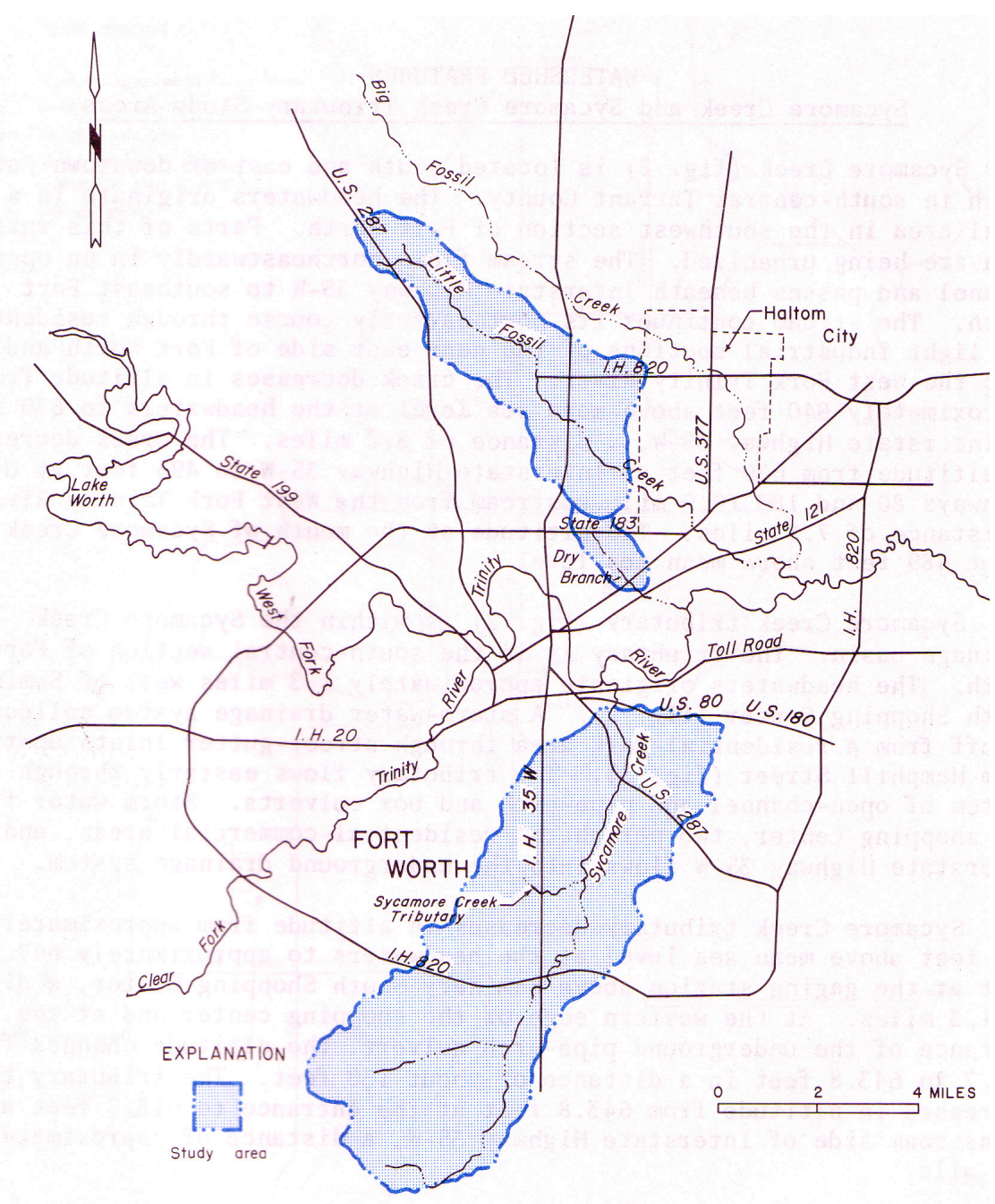

Figure 16. Location map for fortworth database module adopted from Slade, Taylor, and Haynes, 1979.

\section{SAN ANTONIO URBAN STUDIES}

Land, L.F., 1971, Annual compilation and analysis of hydrologic data for urban studies in the San Antonio, Texas metropolitan area, 1969: U.S. Geological Survey 109 p.

Land, L.F., 1972, Annual compilation and analysis of hydrologic data for urban studies in the San Antonio, Texas metropolitan area, 1970: U.S. Geological Survey, Texas District, $178 \mathrm{p}$.

Steger, R.D., 1973, Annual compilation and analysis of hydrologic data for urban studies in the San Antonio, Texas metropolitan area, 1971: U.S. Geological Survey, Texas District, $109 \mathrm{p}$.

Steger, R.D., 1974, Hydrologic data for urban studies in the San Antonio, Texas metropolitan area, 1972: U.S. Geological Survey Open-File Report, 102 p. 
Steger, R.D., 1975, Hydrologic data for urban studies in the San Antonio, Texas metropolitan area, 1973: U.S. Geological Survey Open-File Report, 127 p.

Gonzalez, Victor, 1976, Hydrologic data for urban studies in the San Antonio, Texas metropolitan area, 1974: U.S. Geological Survey Open-File Report, 109 p.

Harmsen, Lynn, 1977, Hydrologic data for urban studies in the San Antonio, Texas metropolitan area, 1975: U.S. Geological Survey Open-File Report 77-221, 91 p.

Harmsen, Lynn, 1978, Hydrologic data for urban studies in the San Antonio, Texas metropolitan area, 1976: U.S. Geological Survey Open-File Report 78-164, 132 p.

Perez, Roberto, 1980, Hydrologic data for urban studies in the San Antonio, Texas metropolitan area, 1977: U.S. Geological Survey Open-File Report 80-743, 100 p.

Perez, Roberto, 1981, Hydrologic data for urban studies in the San Antonio, Texas metropolitan area, 1978: U.S. Geological Survey Open-File Report 81-922, 91 p.

Perez, Roberto, 1982, Hydrologic data for urban studies in the San Antonio, Texas metropolitan area, 1979-80: U.S. Geological Survey Open-File Report 82-158, 125 p.

Perez, Roberto, 1983, Hydrologic data for urban studies in the San Antonio, Texas metropolitan area, 1981: U.S. Geological Survey Open-File Report 83-35, 58 p.

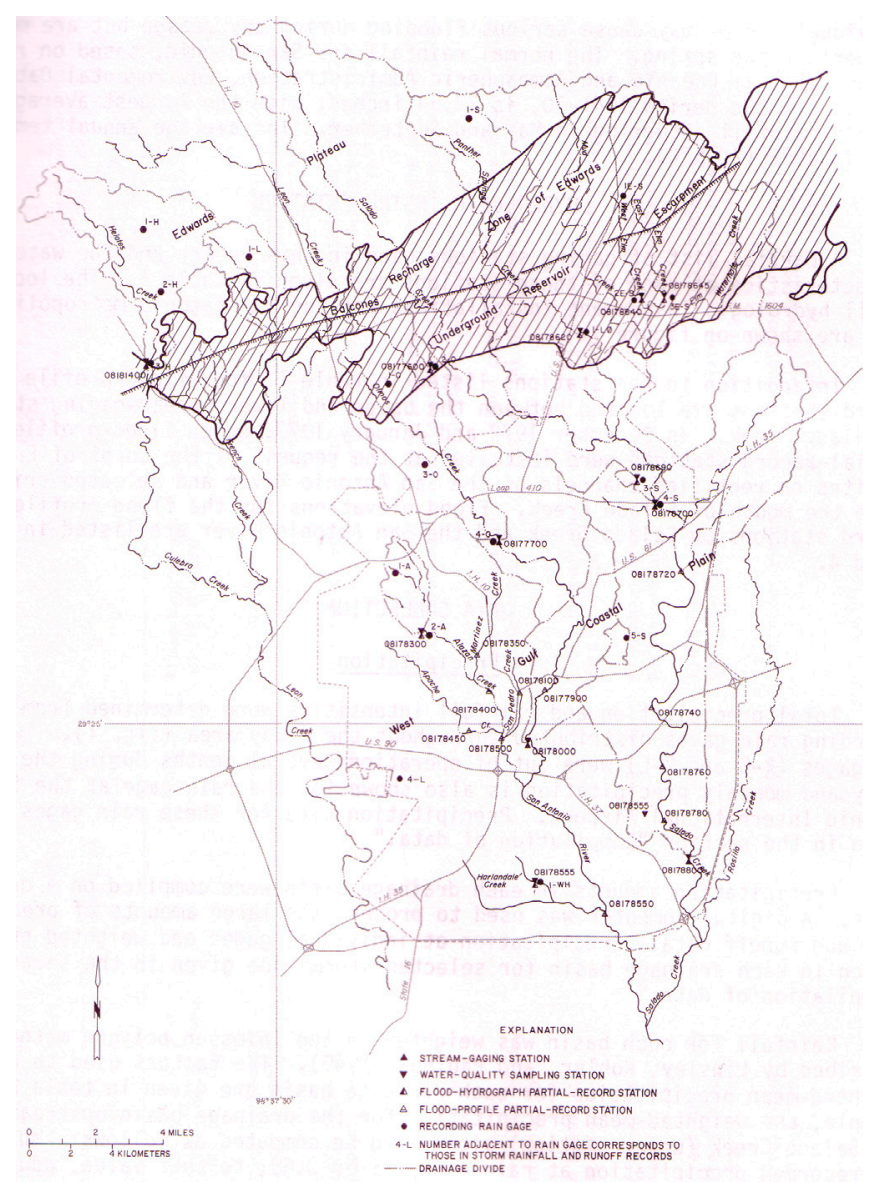

Figure 17. Location map for sanantonio database module adopted from Perez, 1983. 


\section{BRAZOS RIVER BASIN: Cow Bayou Watershed Studies}

U.S. Geological Survey, 1960, Hydrologic studies of small watersheds, Cow Bayou Basin, Texas: U.S. Geological Survey, Texas District, 52 p.

U.S. Geological Survey, 1961, Hydrologic studies of small watersheds, Cow Bayou Basin, Texas: U.S. Geological Survey, Texas District, 17 p.

U.S. Geological Survey, 1962, Hydrologic data of Cow Bayou, Brazos River Basin, Texas, 1962: U.S. Geological Survey, Texas District, 68 p.

U.S. Geological Survey, 1963, Compilation of hydrologic data, Cow Bayou, Brazos River Basin, Texas, 1963: U.S. Geological Survey, Texas District, 40 p.

U.S. Geological Survey, 1964, Compilation of hydrologic data, Cow Bayou, Brazos River Basin, Texas, 1964: U.S. Geological Survey, Texas District, 55 p.

U.S. Geological Survey, 1965, Compilation of hydrologic data, Cow Bayou, Brazos River Basin, Texas, 1965: U.S. Geological Survey, Texas District, 64 p.

U.S. Geological Survey, 1966, Compilation of hydrologic data, Cow Bayou, Brazos River Basin, Texas, 1966: U.S. Geological Survey, Texas District, 72 p.

U.S. Geological Survey, 1967, Compilation of hydrologic data, Cow Bayou, Brazos River Basin, Texas, 1967: U.S. Geological Survey, Texas District, 62 p.

U.S. Geological Survey, 1968, Compilation of hydrologic data, Cow Bayou, Brazos River Basin, Texas, 1968: U.S. Geological Survey, Texas District, 80 p.

U.S. Geological Survey, 1969, Hydrologic studies of small watersheds, Cow Bayou, Brazos River Basin, Texas, 1955-64: U.S. Geological Survey, Texas District, 66 p.

Sansom, J.N., 1969, Annual compilation and analysis of hydrologic data for Cow Bayou, Brazos River Basin, Texas, 1969: U.S. Geological Survey, Texas District, 76 p.

Watson, J.A., 1970, Annual compilation and analysis of hydrologic data for Cow Bayou, Brazos River Basin, Texas, 1970: U.S. Geological Survey, Texas District, 85 p.

Watson, J.A., 1971, Annual compilation and analysis of hydrologic data for Cow Bayou, Brazos River Basin, Texas, 1971: U.S. Geological Survey, Texas District, 63 p.

VanZandt, J.K., 1972, Hydrologic data for Cow Bayou, Brazos River Basin, Texas, 1972: U.S.Geological Survey Open-File Report, 72 p.

VanZandt, J.K., 1973, Hydrologic data for Cow Bayou, Brazos River Basin, Texas, 1973: U.S.Geological Survey Open-File Report, 74 p.

VanZandt, J.K., 1974, Hydrologic data for Cow Bayou, Brazos River Basin, Texas, 1974: U.S.Geological Survey Open-File Report, 63 p.

Mitchell, R.N., and Wehmeyer, E.E., 1977, Hydrologic data for Cow Bayou, Brazos River Basin, Texas, 1975: U.S.Geological Survey Open-File Report 76-723, 81 p. 


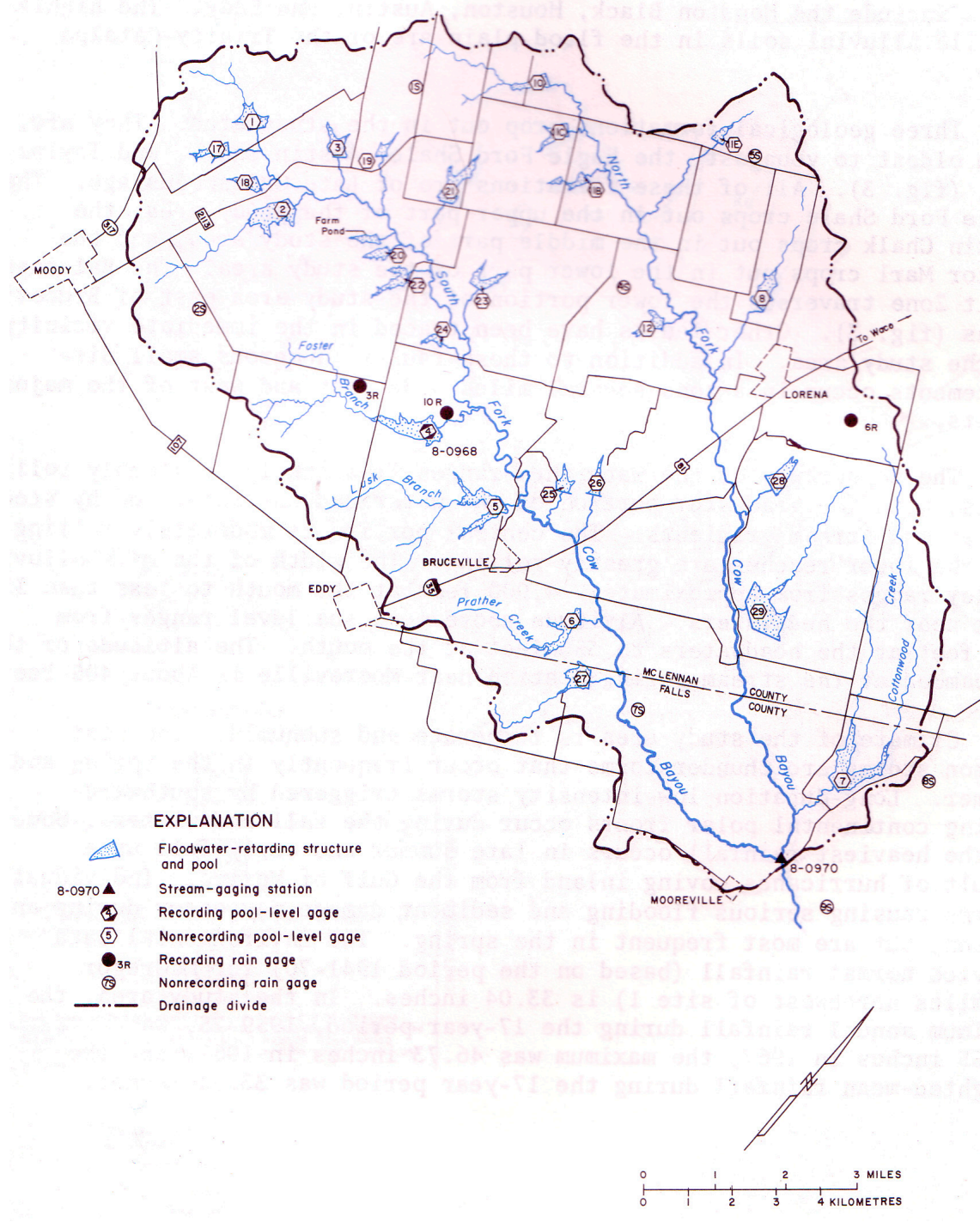

Figure 18. Location map for sma 11 rura 1 sheds database module for Cow Bayou watershed adopted from Mitchell and Wehmeyer, 1977.

\section{BRAZOS RIVER BASIN: Green Creek Watershed Studies}

U.S. Geological Survey, 1960, Hydrologic studies of small watersheds, Green Creek Basin, Texas: U.S. Geological Survey, Texas District, 42 p.

U.S. Geological Survey, 1961, Hydrologic studies of small watersheds, Green Creek study area, Texas, 1961: U.S. Geological Survey, Texas District, [variously paged].

U.S. Geological Survey, 1962, Hydrologic data of Green Creek, Brazos River Basin, Texas, 1962: U.S. Geological Survey, Texas District, [variously paged].

U.S. Geological Survey, 1963, Compilation of hydrologic data, Green Creek, Brazos River Basin, Texas, 1963: U.S. Geological Survey, Texas District, 52 p. 
U.S. Geological Survey, 1964, Compilation of hydrologic data, Green Creek, Brazos River Basin, Texas, 1964: U.S. Geological Survey, Texas District, 58 p.

U.S. Geological Survey, 1965, Compilation of hydrologic data, Green Creek, Brazos River Basin, Texas, 1965: U.S. Geological Survey, Texas District, 44 p.

U.S. Geological Survey, 1966, Compilation of hydrologic data, Green Creek, Brazos River Basin, Texas, 1966: U.S. Geological Survey, Texas District, 109 p.

U.S. Geological Survey, 1967, Compilation of hydrologic data, Green Creek, Brazos River Basin, Texas, 1967: U.S. Geological Survey, Texas District, 34 p.

U.S. Geological Survey, 1968, Compilation of hydrologic data, Green Creek, Brazos River Basin, Texas, 1968: U.S. Geological Survey, Texas District, 66 p.

Massey, B.C., 1969, Annual compilation and analysis of hydrologic data for Green Creek, Brazos River Basin, Texas, 1969: U.S. Geological Survey, Texas District, 44 p.

U.S. Geological Survey, 1969, Preparation of hydrologic data small watersheds project: U.S. Geological Survey, Texas District, 43 p.

Hampton, B.B., 1970, Annual compilation and analysis of hydrologic data for Green Creek, Brazos River Basin, Texas, 1970: U.S. Geological Survey Open-File Report, $42 \mathrm{p}$.

Hampton, B.B., 1971, Annual compilation and analysis of hydrologic data for Green Creek, Brazos River Basin, Texas, 1971: U.S. Geological Survey Open-File Report, $30 \mathrm{p}$.

U.S. Geological Survey, 1972, Hydrologic studies of small watersheds, Green Creek, Brazos River Basin, Texas, 1955-66: U.S. Geological Survey, Texas District, 55 p.

BRAZOS RIVER BASIN: Little Pond Creek and North EIm Creek Watershed Studies

Slade, R.M., Jr., 1970, Annual compilation and analysis of hydrologic data for Little Pond Creek and North Elm Creek, Brazos River Basin, Texas, 1970: U.S. Geological Survey Open-File Report, 44 p.

Hawkinson, R.O., 1972, Hydrologic studies of Little Pond Creek and North Elm Creek watersheds, Brazos River Basin, Texas, 1963-69: U.S. Geological Survey, Texas District, $137 \mathrm{p}$.

VanZandt, J.K., 1973, Annual compilation and analysis of hydrologic data for Little Pond Creek and North Elm Creek, Brazos River Basin, Texas, 1971: U.S. Geological Survey Open-File Report, 52 p.

Mitchell, R.N., 1974, Hydrologic data for Little Pond Creek and North Elm Creek, Brazos River Basin, Texas, 1972: U.S. Geological Survey Open-File Report, 37 p. 


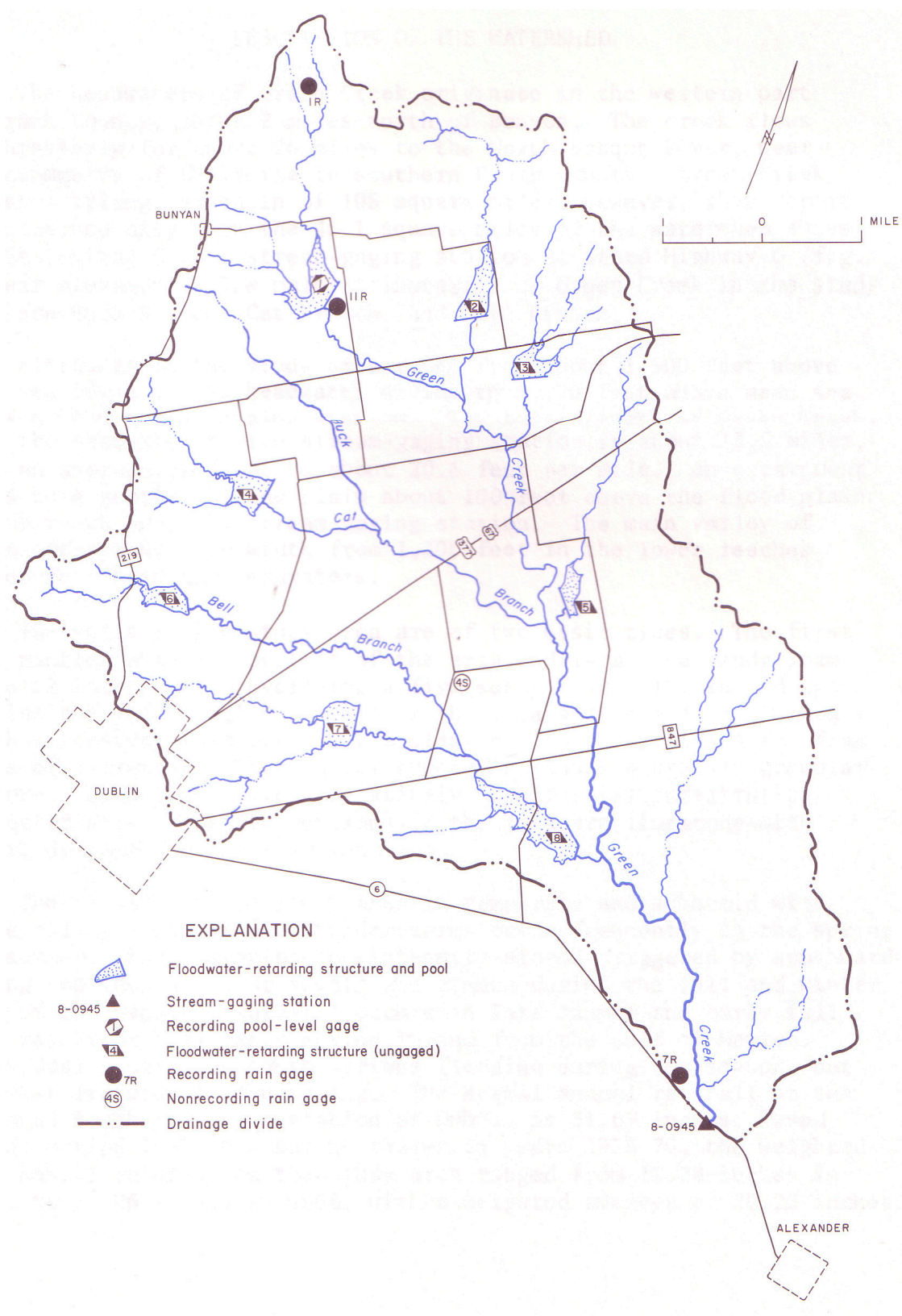

Figure 19. Location map for sma 17 rura 1 sheds database module for Green Creek watershed adopted from Hampton, 1971. 


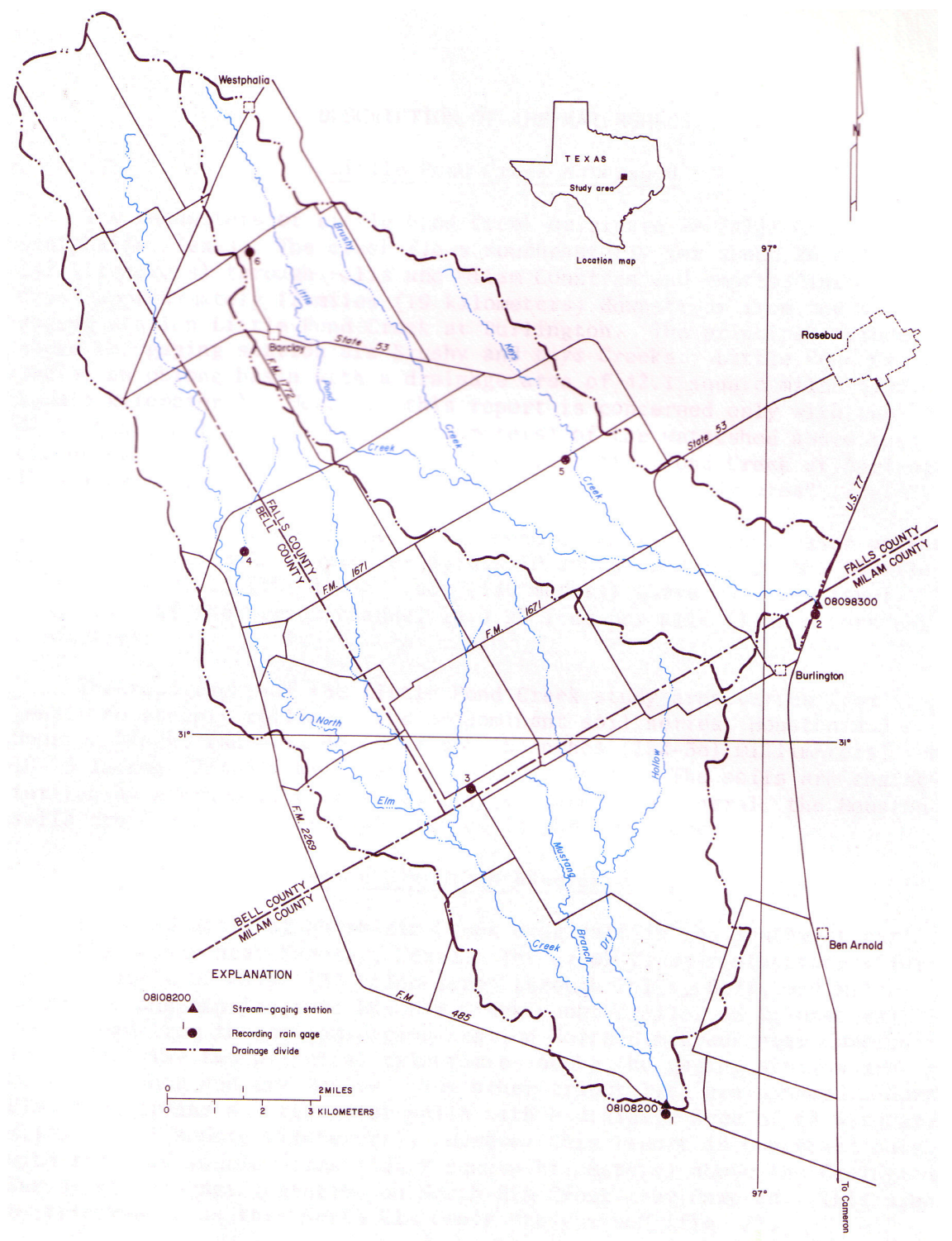

Figure 20. Location map for sma 11 rura 1 sheds database module for Little Pond and North EIm Creek watersheds adopted from Mitchell, 1974.

\section{COLORADO RIVER BASIN: Deep Creek Watershed Studies}

U.S. Geological Survey, 1960, Hydrologic studies of small watersheds, Deep Creek Basin, Texas: U.S. Geological Survey, Texas District, 58 p.

U.S. Geological Survey, 1961, Hydrologic studies of small watersheds, Deep Creek study area, Texas, 1961: U.S. Geological Survey, Texas District, 111 p.

U.S. Geological Survey, 1962, Hydrologic data of Deep Creek, Colorado River Basin, Texas, 1962: U.S. Geological Survey, Texas District, 51 p.

U.S. Geological Survey, 1963, Hydrologic data of Deep Creek, Colorado River Basin, Texas, 1963: U.S. Geological Survey, Texas District, 153 p. 
U.S. Geological Survey, 1964, Compilation of hydrologic data, Deep Creek, Colorado River Basin, Texas, 1964: U.S. Geological Survey, Texas District, 78 p.

U.S. Geological Survey, 1965, Hydrologic studies of small watersheds, Deep Creek, Colorado River Basin, Texas, 1951-61: U.S. Geological Survey, Texas District, 123 p.

U.S. Geological Survey, 1965, Compilation of hydrologic data, Deep Creek, Colorado River Basin, Texas, 1965: U.S. Geological Survey, Texas District, 72 p.

U.S. Geological Survey, 1966, Compilation of hydrologic data, Deep Creek, Colorado River Basin, Texas, 1966: U.S. Geological Survey, Texas District, 55 p.

U.S. Geological Survey, 1967, Compilation of hydrologic data, Deep Creek, Colorado River Basin, Texas, 1967: U.S. Geological Survey, Texas District, 87 p.

U.S. Geological Survey, 1968, Compilation of hydrologic data, Deep Creek, Colorado River Basin, Texas, 1968: U.S. Geological Survey, Texas District, 86 p.

Hejl, H.R., 1971, Annual compilation and analysis of hydrologic data for Deep Creek, Colorado River Basin, Texas, 1969: U.S. Geological Survey, Texas District, 73 p.

Hejl, H.R., 1972, Annual compilation and analysis of hydrologic data for Deep Creek, Colorado River Basin, Texas, 1970: U.S. Geological Survey, Texas District, 35 p.

Lee, J.N., 1973, Annual compilation and analysis of hydrologic data for Deep Creek, Colorado River Basin, Texas, 1971: U.S. Geological Survey, Texas District, 76 p.

\section{COLORADO RIVER BASIN: Mukewater Creek Watershed Studies}

U.S. Geological Survey, 1960, Hydrologic studies of small watersheds Mukewater Creek Basin, Texas: U.S. Geological Survey, Texas District, 30 p.

U.S. Geological Survey, 1961, Hydrologic studies of small watersheds Mukewater Creek study area, Texas, 1961: U.S. Geological Survey, Texas District, 47 p.

U.S. Geological Survey, 1962, Hydrologic data of Mukewater Creek, Colorado River Basin, Texas, 1962: U.S. Geological Survey, Texas District, 60 p.

U.S. Geological Survey, 1963, Compilation of hydrologic data of small watersheds Mukewater Creek, Colorado River Basin, Texas, 1963: U.S. Geological Survey, Texas District, $80 \mathrm{p}$.

U.S. Geological Survey, 1964, Compilation of hydrologic data of small watersheds Mukewater Creek, Colorado River Basin, Texas, 1964: U.S. Geological Survey, Texas District, $56 \mathrm{p}$.

U.S. Geological Survey, 1965, Hydrologic studies of small watersheds Mukewater Creek, Colorado River Basin, Texas, 1952-60: U.S. Geological Survey, Texas District, 70 p.

U.S. Geological Survey, 1965, Compilation of hydrologic data of small watersheds Mukewater Creek, Colorado River Basin, Texas, 1965: U.S. Geological Survey, Texas District, $67 \mathrm{p}$. 


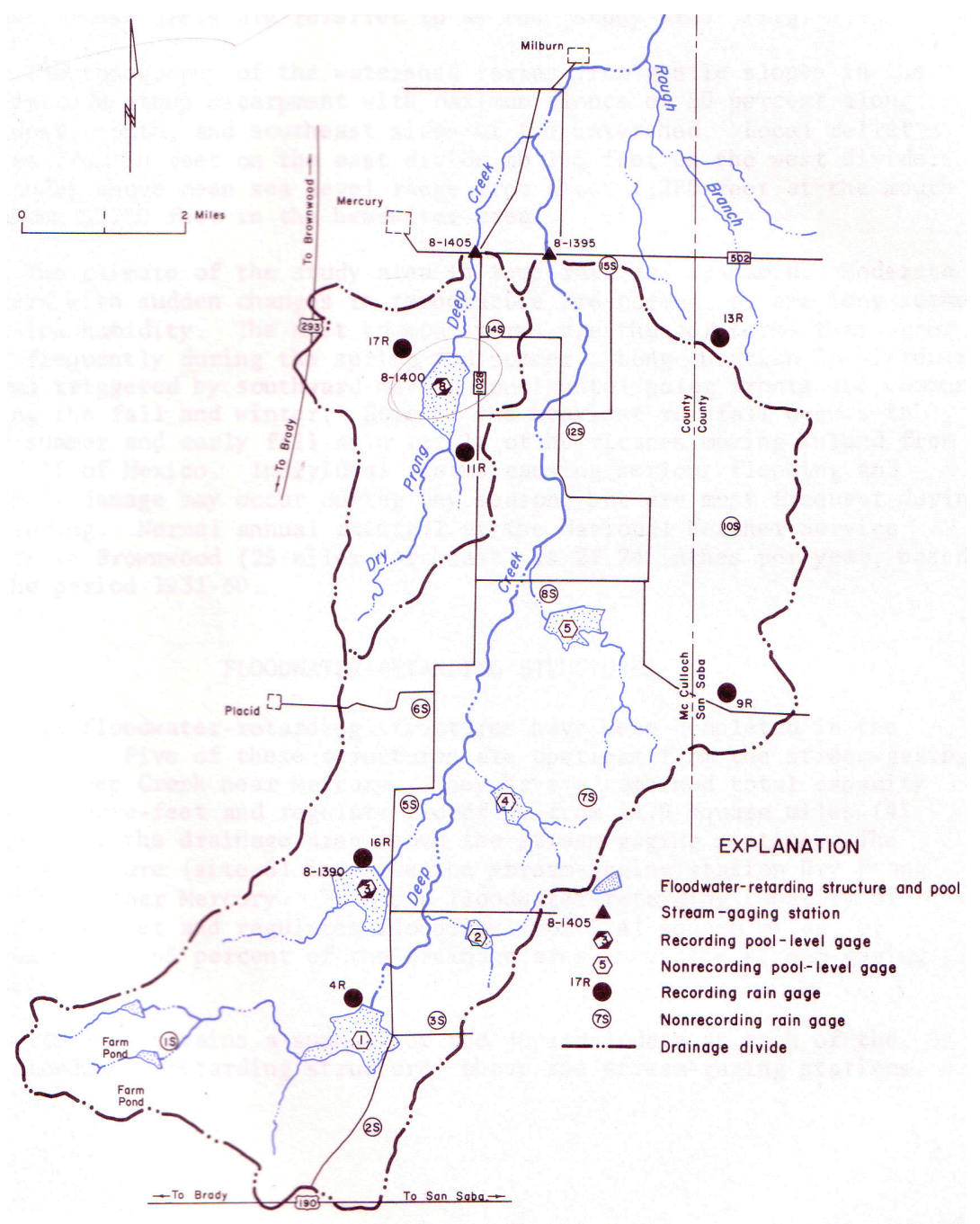

Figure 21. Location map for sma 11 rura 1 sheds database module for Deep Creek watershed adopted from Hejl, 1972.

U.S. Geological Survey, 1966, Compilation of hydrologic data of small watersheds Mukewater Creek, Colorado River Basin, Texas, 1966: U.S. Geological Survey, Texas District, $56 \mathrm{p}$.

U.S. Geological Survey, 1967, Compilation of hydrologic data of small watersheds Mukewater Creek, Colorado River Basin, Texas, 1967: U.S. Geological Survey, Texas District, $87 \mathrm{p}$.

U.S. Geological Survey, 1968, Compilation of hydrologic data of small watersheds Mukewater Creek, Colorado River Basin, Texas, 1968: U.S. Geological Survey, Texas District, $81 \mathrm{p}$.

Hejl, H.R., 1969, Annual compilation and analysis of hydrologic data for Mukewater Creek, Colorado River Basin, Texas, 1969: U.S. Geological Survey, Texas District, $84 \mathrm{p}$. 
Hejl, H.R., 1972, Annual compilation and analysis of hydrologic data for Mukewater Creek, Colorado River Basin, Texas, 1970: U.S. Geological Survey Open-File Report, $69 \mathrm{p}$.

Lee, J.N., 1973, Annual compilation and analysis of hydrologic data for Mukewater Creek, Colorado River Basin, Texas, 1971: U.S. Geological Survey Open-File Report, $89 \mathrm{p}$.

Lee, J.N., 1974, Hydrologic data for Mukewater Creek, Colorado River Basin, Texas, 1972: U.S. Geological Survey Open-File Report, 67 p.

Lee, J.N., 1975, Hydrologic data for Mukewater Creek, Colorado River Basin, Texas, 1973: U.S. Geological Survey Open-File Report, 65 p.

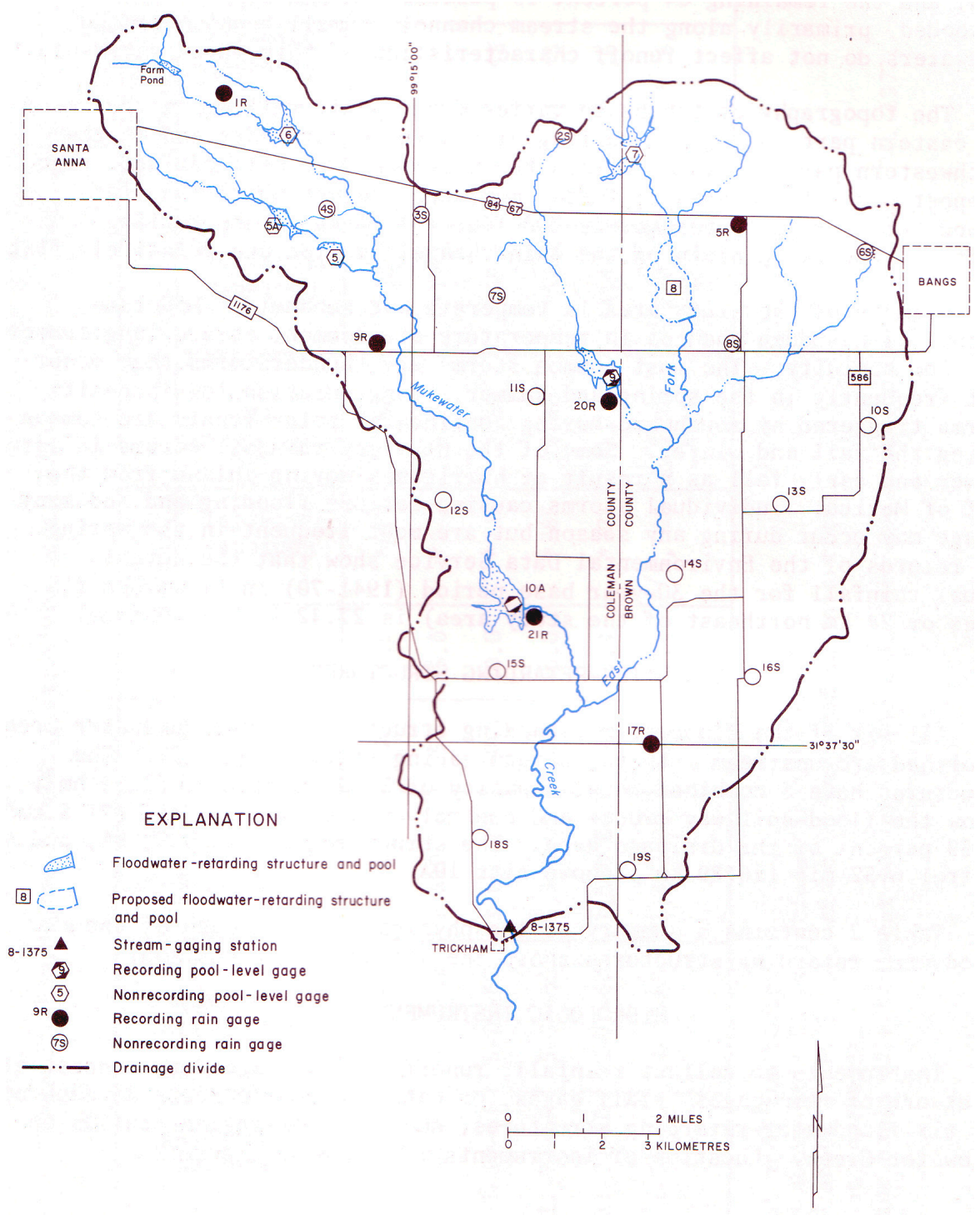

Figure 22. Location map for sma 11 rura 1 sheds database module for Mukewater Creek watershed adopted from Lee, 1975. 
SAN ANTONIO RIVER BASIN: Calaveras Creek Watershed Studies

U.S. Geological Survey, 1960, Hydrologic studies of small watersheds, Calaveras Creek Basin, Texas, 1960: U.S. Geological Survey, Texas District, 36 p.

U.S. Geological Survey, 1961, Hydrologic studies of small watersheds, Calaveras Creek study area, Texas, 1961: U.S. Geological Survey, Texas District, [variously paged].

U.S. Geological Survey, 1962, Hydrologic data of Calaveras Creek, San Antonio River Basin, Texas, 1962: U.S. Geological Survey, Texas District, 41 p.

U.S. Geological Survey, 1963, Compilation of hydrologic data, Calaveras Creek, San Antonio River Basin, Texas, 1963: U.S. Geological Survey, Texas District, 41 p.

U.S. Geological Survey, 1964, Compilation of hydrologic data, Calaveras Creek, San Antonio River Basin, Texas, 1964: U.S. Geological Survey, Texas District, 50 p.

U.S. Geological Survey, 1965, Compilation of hydrologic data, Calaveras Creek, San Antonio River Basin, Texas, 1965: U.S. Geological Survey, Texas District, 62 p.

U.S. Geological Survey, 1966, Compilation of hydrologic data, Calaveras Creek, San Antonio River Basin, Texas, 1966: U.S. Geological Survey, Texas District, 34 p.

U.S. Geological Survey, 1967, Compilation of hydrologic data, Calaveras Creek, San Antonio River Basin, Texas, 1967: U.S. Geological Survey, Texas District, 47 p.

U.S. Geological Survey, 1968, Compilation of hydrologic data, Calaveras Creek, San Antonio River Basin, Texas, 1968: U.S. Geological Survey, Texas District, 66 p.

Alexander, J.M., 1969, Annual compilation and analysis of hydrologic data for Calaveras Creek, San Antonio River Basin, Texas, 1969: U.S. Geological Survey, Texas District, $56 \mathrm{p}$.

Reddy, D.R., 1971, Annual compilation and analysis of hydrologic data for Calaveras Creek, San Antonio River Basin, Texas, 1970: U.S. Geological Survey Open-File Report, 63 p.

U.S. Geological Survey, 1972, Hydrologic studies of small watersheds, Calaveras Creek, San Antonio River Basin, Texas, 1955-68: U.S. Geological Survey, Texas District, $109 \mathrm{p}$.

Steger, R.D., 1973, Annual compilation and analysis of hydrologic data for Calaveras and Escondido Creeks, San Antonio River Basin, Texas, 1971: U.S. Geological Survey Open-File Report, 74 p. 


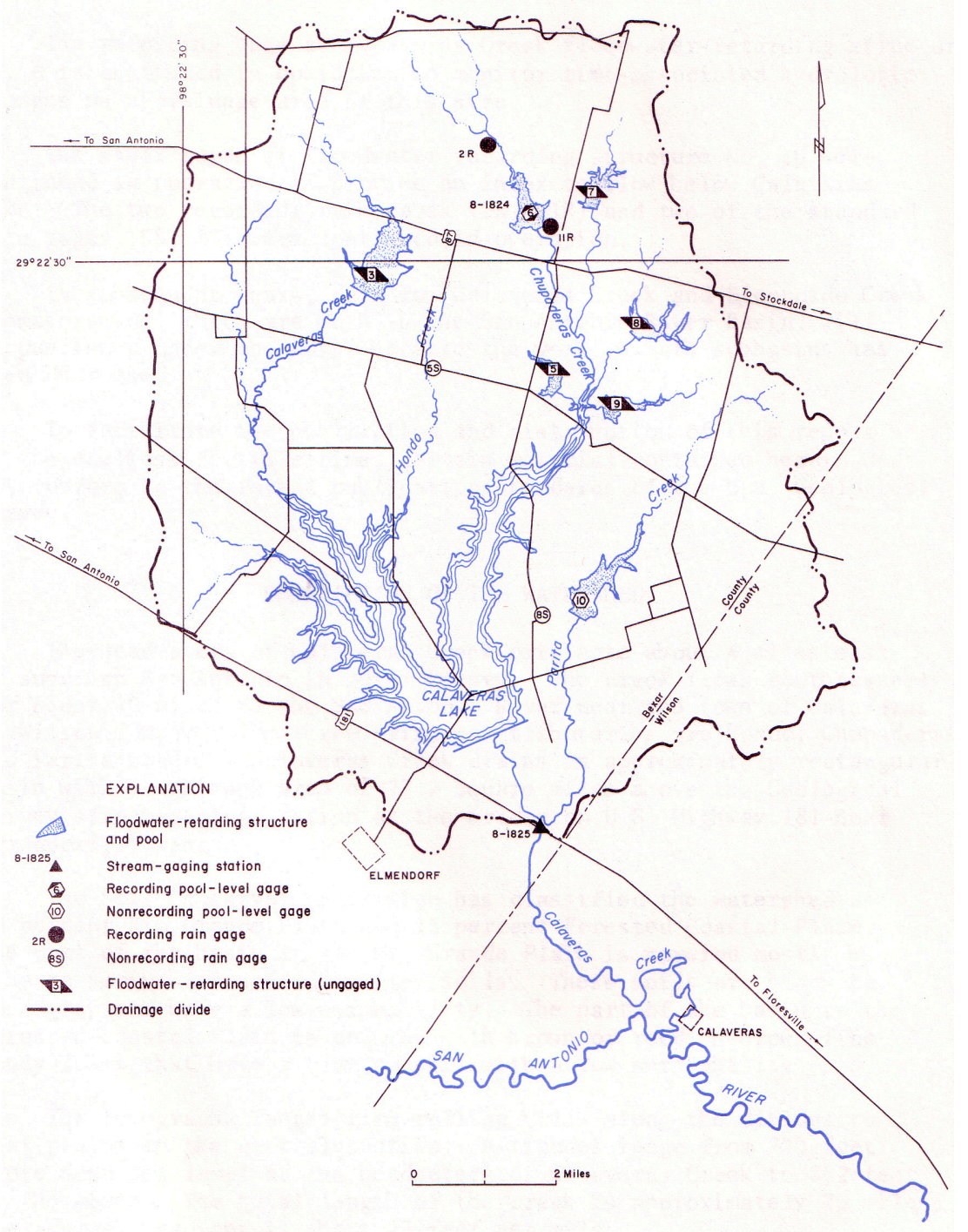

Figure 23. Location map for sma 11 rura 1 sheds database module for Calaveras Creek watershed adopted from Reddy, 1971.

\section{SAN ANTONIO RIVER BASIN: Escondido Creek Watershed Studies}

U.S. Geological Survey, 1960, Hydrologic studies of small watersheds, Escondido Creek Basin, Texas, 1960: U.S. Geological Survey, Texas District, 45 p.

U.S. Geological Survey, 1961, Hydrologic studies of small watersheds, Escondido Creek Basin, Texas, 1961: U.S. Geological Survey, Texas District, 70 p.

U.S. Geological Survey, 1962, Hydrologic data of Escondido Creek, San Antonio River Basin, Texas, 1962: U.S. Geological Survey, Texas District, 43 p.

U.S. Geological Survey, 1963, Compilation of hydrologic data, Escondido Creek, San Antonio River Basin, Texas, 1963: U.S. Geological Survey, Texas District, 85 p. 
U.S. Geological Survey, 1964, Compilation of hydrologic data, Escondido Creek, San Antonio River Basin, Texas, 1964: U.S. Geological Survey, Texas District, 86 p.

U.S. Geological Survey, 1965, Compilation of hydrologic data, Escondido Creek, San Antonio River Basin, Texas, 1965: U.S. Geological Survey, Texas District, 72 p.

U.S. Geological Survey, 1966, Compilation of hydrologic data, Escondido Creek, San Antonio River Basin, Texas, 1966: U.S. Geological Survey, Texas District, 44 p.

U.S. Geological Survey, 1967, Hydrologic studies of small watersheds, Escondido Creek, San Antonio River Basin, Texas, 1955-63: U.S. Geological Survey, Texas District, $123 \mathrm{p}$.

U.S. Geological Survey, 1967, Compilation of hydrologic data, Escondido Creek, San Antonio River Basin, Texas, 1967: U.S. Geological Survey, Texas District, 73 p.

U.S. Geological Survey, 1968, Compilation of hydrologic data, Escondido Creek, San Antonio River Basin, Texas, 1968: U.S. Geological Survey, Texas District, 73 p.

Reddy, D.R., 1971, Annual compilation and analysis of hydrologic data for Escondido Creek, San Antonio River Basin Texas, 1969: U.S. Geological Survey, Texas District, $62 \mathrm{p}$.

Reddy, D.R., 1971, Annual compilation and analysis of hydrologic data for Escondido Creek, San Antonio River Basin, Texas, 1970: U.S. Geological Survey Open-File Report, 65 p.

Steger, R.D., 1973, Annual compilation and analysis of hydrologic data for Calaveras and Escondido Creeks, San Antonio River Basin, Texas, 1971: U.S. Geological Survey Open-File Report, 74 p. [also listed in SAN ANTONIO RIVER BASIN: Calaveras Creek Watershed Studies section]

\section{TRINITY RIVER BASIN: EIm Fork Trinity River Watershed Studies}

U.S. Geological Survey, 1961, Hydrologic studies of small watersheds, Elm Fork Trinity River study area, Texas, 1961: U.S. Geological Survey, Texas District, 43 p.

U.S. Geological Survey, 1962, Hydrologic studies of small watersheds, Elm Fork Trinity River Basin, Montague and Cooke Counties, Texas 1956-60: U.S. Geological Survey, Texas District, 77 p.

U.S. Geological Survey, 1962, Hydrologic data of Elm Fork Trinity River, Trinity River Basin, Texas, 1962: U.S. Geological Survey, Texas District, 60 p.

U.S. Geological Survey, 1963, Compilation of hydrologic data, Elm Fork Trinity River, Trinity River Basin, Texas, 1963: U.S. Geological Survey, Texas District, $60 \mathrm{p}$.

U.S. Geological Survey, 1965, Compilation of hydrologic data, Elm Fork Trinity River, Trinity River Basin, Texas, 1964-65: U.S. Geological Survey, Texas District, 135 p. 


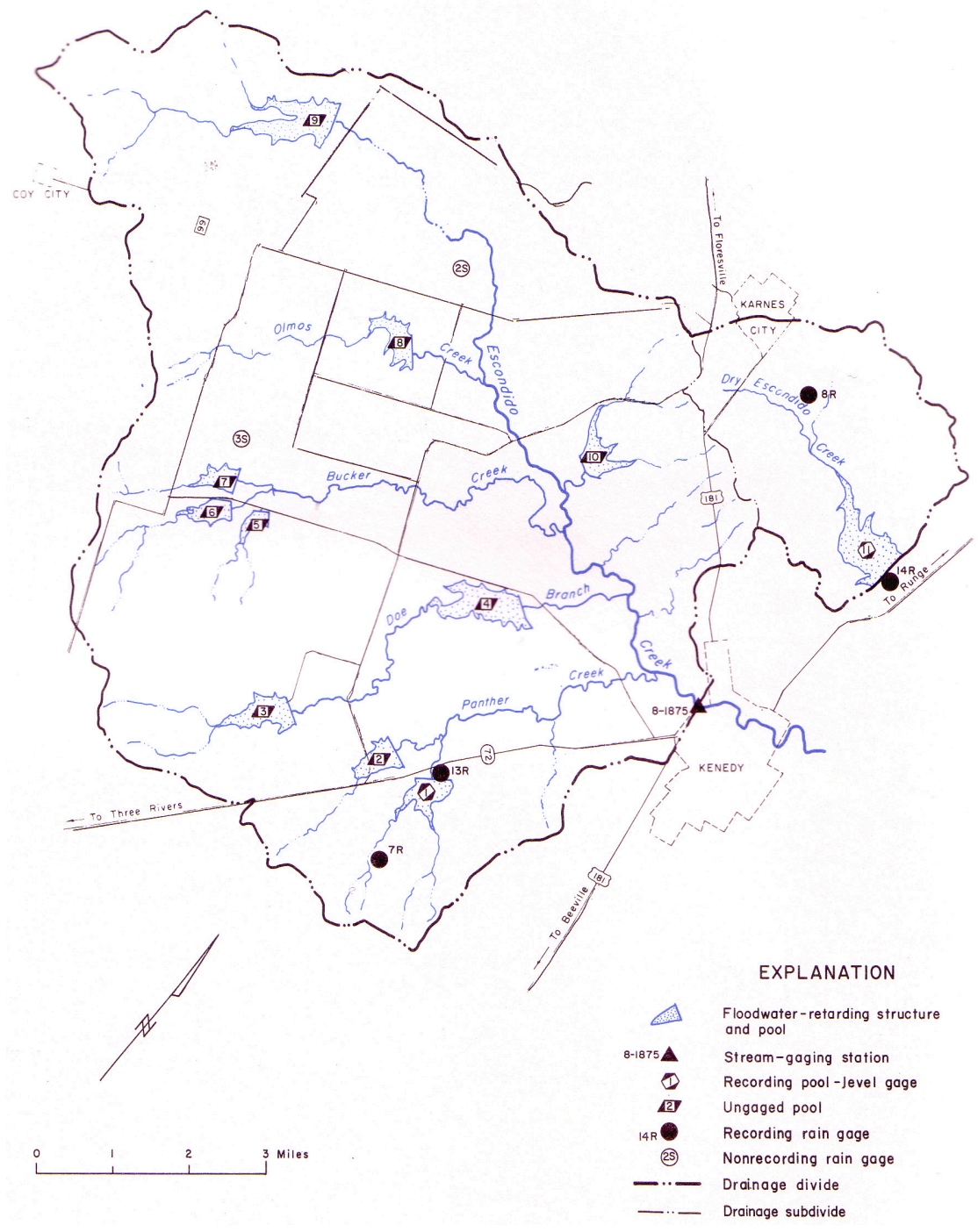

Figure 24. Location map for sma 11 rura 1 sheds database module for Escondido Creek watershed adopted from Steger, 1973.

U.S. Geological Survey, 1966, Compilation of hydrologic data, Elm Fork Trinity River, Trinity River Basin, Texas, 1966: U.S. Geological Survey, Texas District, 60 p.

U.S. Geological Survey, 1967, Compilation of hydrologic data, Elm Fork Trinity River, Trinity River Basin, Texas, 1967: U.S. Geological Survey, Texas District, 54 p.

U.S. Geological Survey, 1968, Compilation of hydrologic data, Elm Fork Trinity River, Trinity River Basin, Texas, 1968: U.S. Geological Survey, Texas District, 84 p.

Sansom, J.N., 1969, Annual compilation and analysis of hydrologic data for Elm Fork Trinity River, Trinity River Basin, Texas, 1969: U.S. Geological Survey, Texas District, $47 \mathrm{p}$. 
Sansom, J.N., 1972, Annual compilation and analysis of hydrologic data for Elm Fork Trinity River, Trinity River Basin, Texas, 1970: U.S. Geological Survey Open-File Report, 47 p.

Lucero, E.D., 1973, Annual compilation and analysis of hydrologic data for Elm Fork Trinity River, Trinity River Basin, Texas, 1971: U.S. Geological Survey Open-File Report, 27 p.

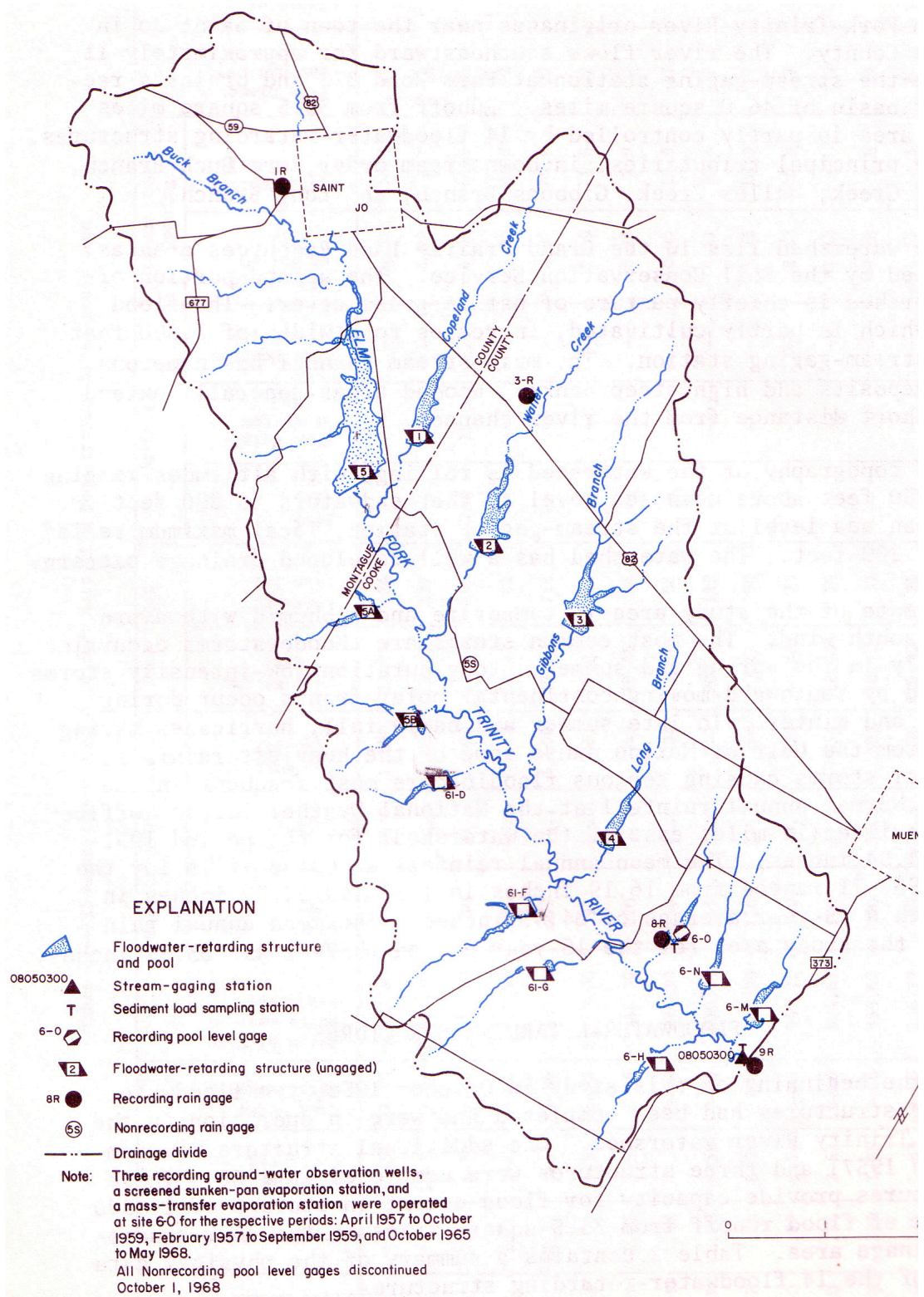

Figure 25. Location map for sma 11 rura 1 sheds database module for Elm Fork Trinity River watershed adopted from Lucero, 1973. 


\section{TRINITY RIVER BASIN: Honey Creek Watershed Studies}

U.S. Geological Survey, 1960, Hydrologic studies of small watersheds, Honey Creek Basin, Texas, 1960: U.S. Geological Survey, Texas District, 89 p.

U.S. Geological Survey, 1961, Hydrologic studies of small watersheds, Honey Creek Basin, Texas, 1961: U.S. Geological Survey, Texas District, 73 p.

U.S. Geological Survey, 1962, Hydrologic studies of small watersheds, Honey Creek Basin, Collin and Grayson Counties, Texas, 1953-59: U.S. Geological Survey, Texas District, $102 \mathrm{p}$.

U.S. Geological Survey, 1962, Hydrologic data of Honey Creek, Trinity River Basin, Texas, 1962: U.S. Geological Survey, Texas District, 148 p.

U.S. Geological Survey, 1963, Compilation of hydrologic data, Honey Creek, Trinity River Basin, Texas, 1963: U.S. Geological Survey, Texas District, 83 p.

U.S. Geological Survey, 1964, Compilation of hydrologic data, Honey Creek, Trinity River Basin, Texas, 1964: U.S. Geological Survey, Texas District, 77 p.

U.S. Geological Survey, 1965, Compilation of hydrologic data, Honey Creek, Trinity River basin Texas, 1965: U.S. Geological Survey, Texas District, 79 p.

U.S. Geological Survey, 1966, Compilation of hydrologic data, Honey Creek, Trinity River Basin, Texas, 1966: U.S. Geological Survey, Texas District, 126 p.

U.S. Geological Survey, 1967, Compilation of hydrologic data, Honey Creek, Trinity River Basin, Texas, 1967: U.S. Geological Survey, Texas District, 68 p.

U.S. Geological Survey, 1968, Compilation of hydrologic data, Honey Creek, Trinity River Basin, Texas, 1968: U.S. Geological Survey, Texas District, 78 p.

Sansom, J.N., 1969, Annual compilation and analysis of hydrologic data, Honey Creek, Trinity River Basin, Texas, 1969: U.S. Geological Survey Open-File Report, 85 p.

Sansom, J.N., 1972, Annual compilation and analysis of hydrologic data, Honey Creek, Trinity River Basin, Texas, 1970: U.S. Geological Survey Open-File Report, 66 p.

Hampton, B.B., 1973, Annual compilation and analysis of hydrologic data for Honey Creek, Trinity River Basin, Texas, 1971: U.S. Geological Survey Open-File Report, $28 \mathrm{p}$. 


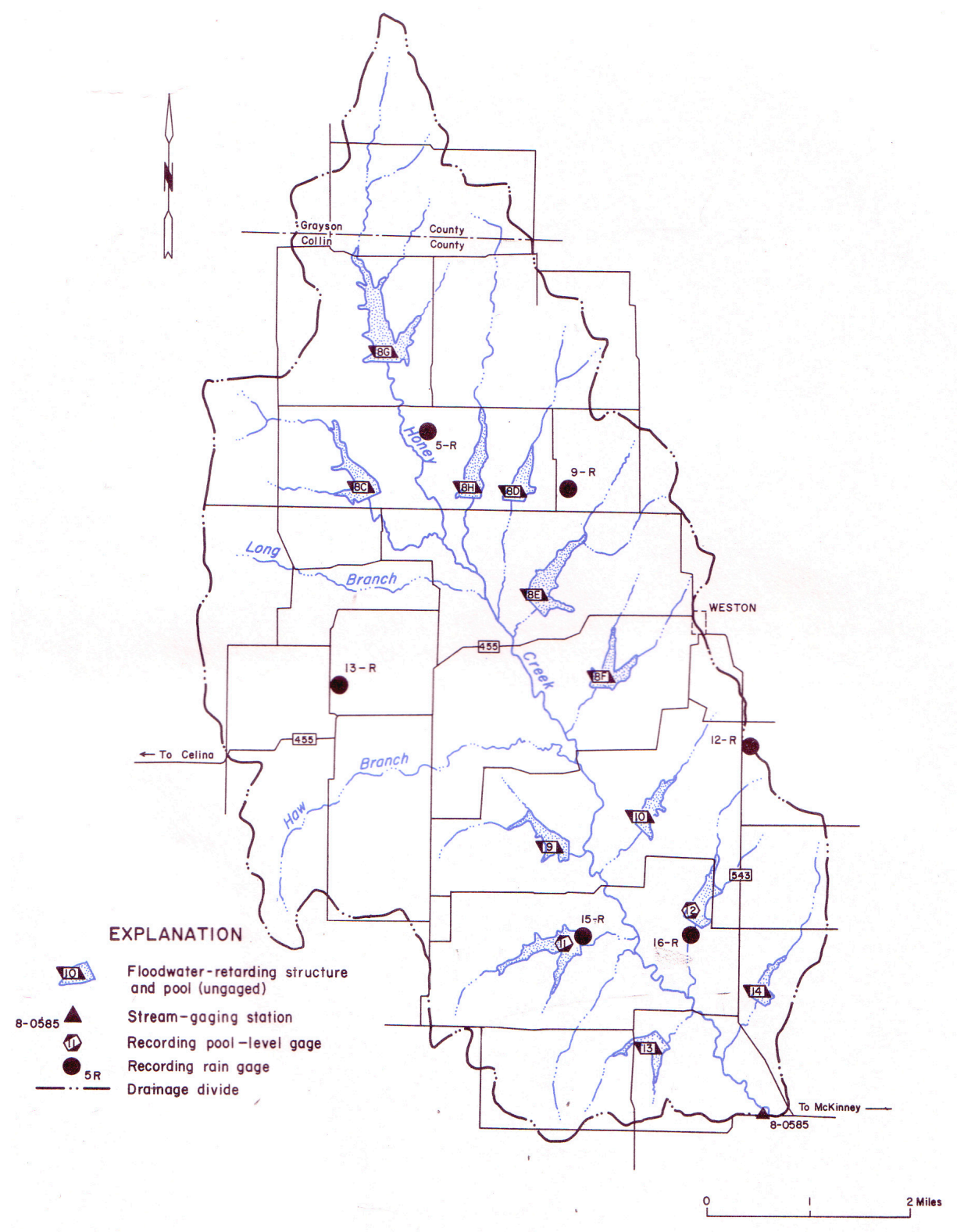

Figure 26. Location map for sma 11 rura 1 sheds database module for Honey Creek watershed adopted from Hampton, 1973.

TRINITY RIVER BASIN: Little EIm Creek Watershed Studies

U.S. Geological Survey, 1960, Hydrologic studies of small watersheds, Little Elm Creek Basin, Texas, 1960: U.S. Geological Survey, Texas District, 27 p.

U.S. Geological Survey, 1961, Hydrologic studies of small watersheds, Little Elm Creek study area, Trinity River Basin, Texas, 1961: U.S. Geological Survey, Texas District, $23 \mathrm{p}$.

U.S. Geological Survey, 1962, Hydrologic data of Little Elm Creek, Trinity River Basin, Texas, 1962: U.S. Geological Survey, Texas District, 35 p. 
U.S. Geological Survey, 1963, Compilation of hydrologic data, Little Elm Creek, Trinity River Basin, Texas, 1963: U.S. Geological Survey, Texas District, 34 p.

U.S. Geological Survey, 1964, Compilation of hydrologic data, Little Elm Creek, Trinity River Basin, Texas, 1964: U.S. Geological Survey, Texas District, 28 p.

U.S. Geological Survey, 1965, Compilation of hydrologic data, Little Elm Creek, Trinity River Basin, Texas, 1965: U.S. Geological Survey, Texas District, 28 p.

U.S. Geological Survey, 1966, Hydrologic studies of small watersheds, Little Elm Creek, Trinity River Basin, Texas, 1956-62: U.S. Geological Survey, Texas District, 59 p.

U.S. Geological Survey, 1966, Compilation of hydrologic data, Little Elm Creek, Trinity River Basin, Texas, 1966: U.S. Geological Survey, Texas District, 69 p.

U.S. Geological Survey, 1967, Compilation of hydrologic data, Little Elm Creek, Trinity River Basin, Texas, 1967: U.S. Geological Survey, Texas District, 81 p.

U.S. Geological Survey, 1968, Compilation of hydrologic data, Little Elm Creek, Trinity River Basin, Texas, 1968: U.S. Geological Survey, Texas District, 83 p.

Hampton, B.B., 1971, Annual compilation and analysis of hydrologic data for Little Elm Creek, Trinity River Basin, Texas, 1969: U.S. Geological Survey, Texas District, 68 p.

Hampton, B.B., 1972, Annual compilation and analysis of hydrologic data for Little Elm Creek, Trinity River Basin, Texas, 1970: U.S. Geological Survey, Texas District, $100 \mathrm{p}$.

Hampton, B.B., 1973, Annual compilation and analysis of hydrologic data for Little Elm Creek, Trinity River Basin, Texas, 1971: U.S. Geological Survey, Texas District, 47 p.

Hampton, B.B., 1974, Hydrologic data for Little Elm Creek, Trinity River Basin, Texas, 1972: U.S. Geological Survey Open-File Report, 74 p.

Slade, R.M., Jr., and Taylor, J.M., 1975, Hydrologic data for Little Elm Creek, Trinity River Basin, Texas, 1973: U.S. Geological Survey Open-File Report, 74 p.

Slade, R.M., Jr., and Taylor, J.M., 1976, Hydrologic data for Little Elm Creek, Trinity River Basin, Texas, 1974: U.S. Geological Survey Open-File Report, 73 p.

Slade, R.M., Jr., and Taylor, J.M., 1977, Hydrologic data for Little Elm Creek, Trinity River Basin, Texas, 1975: U.S. Geological Survey Open-File Report 77-83, 108 p.

Slade, R.M., Jr., and Taylor, J.M., 1978, Hydrologic data for Little Elm Creek, Trinity River Basin, Texas, 1976: U.S. Geological Survey Open-File Report 78-100, 78 p.

\section{TRINITY RIVER BASIN: North Creek Watershed Studies}

U.S. Geological Survey, 1960, Hydrologic studies of small watersheds, North Creek Basin, Texas: U.S. Geological Survey, Texas District, 19 p.

U.S. Geological Survey, 1961, Hydrologic studies of small watersheds, North Creek study area, Texas: U.S. Geological Survey, Texas District, [variously paged]. 


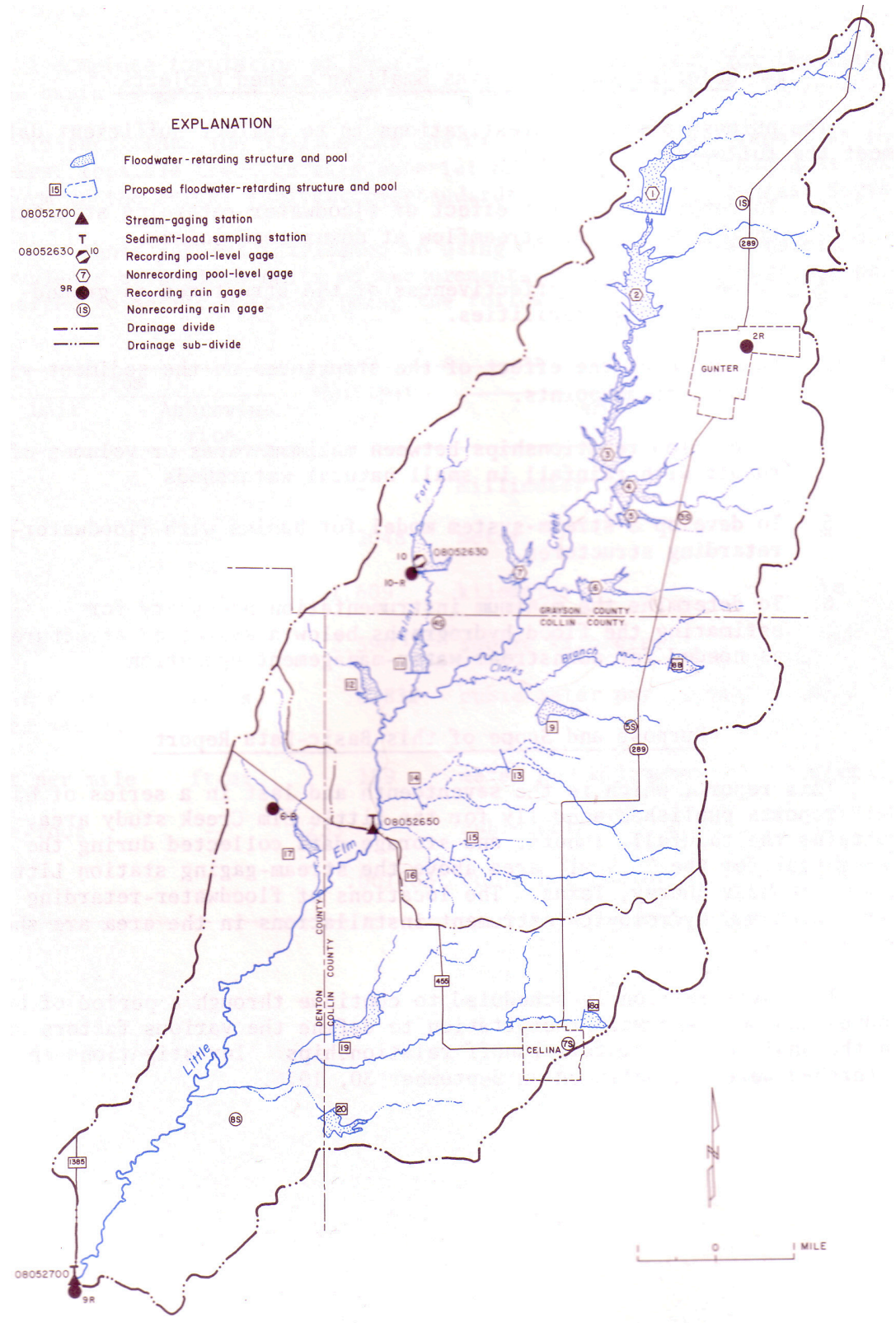

Figure 27. Location map for sma 11 rura 1 sheds database module for Little EIm Creek watershed adopted from Slade and Taylor, 1978.

U.S. Geological Survey, 1963, Compilation of hydrologic data, North Creek, Trinity River Basin, Texas, 1962-63: U.S. Geological Survey, Texas District, 47 p.

U.S. Geological Survey, 1964, Compilation of hydrologic data, North Creek, Trinity River Basin, Texas, 1964: U.S. Geological Survey, Texas District, 19 p.

U.S. Geological Survey, 1965, Compilation of hydrologic data, North Creek, Trinity River Basin, Texas, 1965: U.S. Geological Survey, Texas District, 30 p. 
U.S. Geological Survey, 1966, Compilation of hydrologic data, North Creek, Trinity River Basin, Texas, 1966: U.S. Geological Survey, Texas District, 28 p.

U.S. Geological Survey, 1967, Compilation of hydrologic data, North Creek, Trinity River Basin, Texas, 1967: U.S. Geological Survey, Texas District, 27 p.

U.S. Geological Survey, 1968, Compilation of hydrologic data, North Creek, Trinity River Basin, Texas, 1968: U.S. Geological Survey, Texas District, 36 p.

Kidwell, C.C., 1969, Annual compilation and analysis of hydrologic data for North Creek, Trinity River Basin, Texas, 1969: U.S. Geological Survey, Texas District, 32 p.

Maderak, M.L. and Lucero, E.D., 1971, Annual compilation and analysis of hydrologic data for North Creek, Trinity River Basin, Texas, 1970: U.S. Geological Survey OpenFile Report, $32 \mathrm{p}$.

Lucero, E.D., 1973, Annual compilation and analysis of hydrologic data for North Creek, Trinity River Basin, Texas, 1971: U.S. Geological Survey Open-File Report, 23 p.

Slade, R.M., Jr., 1974, Hydrologic data for North Creek Trinity River Basin, Texas, 1972: U.S. Geological Survey Open-File Report, 31 p.

Slade, R.M., Jr., 1975, Hydrologic data for North Creek Trinity River Basin, Texas, 1973: U.S. Geological Survey Open-File Report, 44 p.

Slade, R.M., Jr., 1976, Hydrologic data for North Creek Trinity River Basin, Texas, 1974: U.S. Geological Survey Open-File Report, 40 p.

Kidwell, C.C., 1977, Hydrologic data for North Creek Trinity River Basin, Texas, 1975: U.S. Geological Survey Open-File Report 76-724, 50 p.

Kidwell, C.C., 1978, Hydrologic data for North Creek Trinity River Basin, Texas, 1976: U.S. Geological Survey Open-File Report 77-732, 42 p.

Kidwell, C.C., 1979, Hydrologic data for North Creek Trinity River Basin, Texas, 1977: U.S. Geological Survey Open-File Report 79-335, 39 p.

Kidwell, C.C., 1980, Hydrologic data for North Creek Trinity River Basin, Texas, 1978: U.S. Geological Survey Open-File Report 80-573, 44 p.

Kidwell, C.C., 1981, Hydrologic data for North Creek Trinity River Basin, Texas, 1979: U.S. Geological Survey Open-File Report 81-823, 38 p.

\section{TRINITY RIVER BASIN: Pin Oak Creek Watershed Studies}

U.S. Geological Survey, 1960, Hydrologic studies of small watersheds, Pin Oak Creek, Trinity River Basin, Texas, 1956-62: U.S. Geological Survey, Texas District, 69 p.

U.S. Geological Survey, 1960, Hydrologic studies of small watersheds, Pin Oak Creek Basin, Texas, 1960: U.S. Geological Survey, Texas District, 44 p.

U.S. Geological Survey, 1961, Hydrologic studies of small watersheds, Pin Oak Creek study area, Texas 1961: U.S. Geological Survey, Texas District, 38 p. 


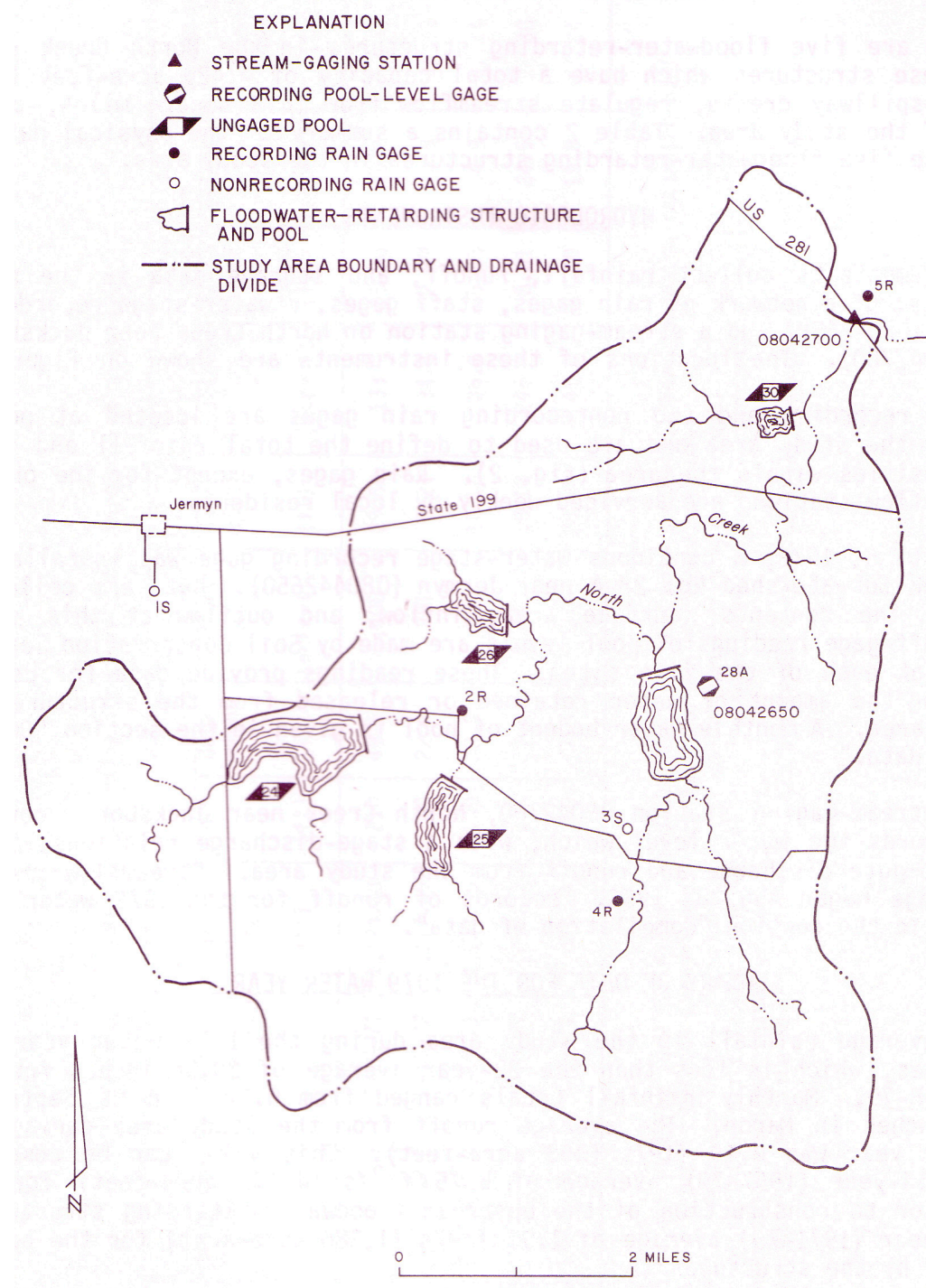

Figure 28. Location map for sma 11 rura 1 sheds database module for North Creek watershed adopted from Kidwell, 1979.

U.S. Geological Survey, 1964, Compilation of hydrologic data, Pin Oak Creek, Trinity River Basin, Texas, 1962-64: U.S. Geological Survey, Texas District, 36 p.

U.S. Geological Survey, 1965, Compilation of hydrologic data, Pin Oak Creek, Trinity River Basin, Texas, 1965: U.S. Geological Survey, Texas District, 30 p.

U.S. Geological Survey, 1966, Compilation of hydrologic data, Pin Oak Creek, Trinity River Basin, Texas, 1966: U.S. Geological Survey, Texas District, 27 p.

U.S. Geological Survey, 1967, Compilation of hydrologic data, Pin Oak Creek, Trinity River Basin, Texas, 1967: U.S. Geological Survey, Texas District, 25 p.

U.S. Geological Survey, 1968, Compilation of hydrologic data, Pin Oak Creek, Trinity River Basin Texas, 1968: U.S. Geological Survey, Texas District, 44 p. 
Hampton, B.B., and Myers, D.R., 1969, Annual compilation and analysis of hydrologic data, Pin Oak Creek, Trinity River Basin Texas, 1969: U.S. Geological Survey, Texas District, $28 \mathrm{p}$.

Hampton, B.B., and Myers, D.R., 1972, Annual compilation and analysis of hydrologic data, Pin Oak Creek, Trinity River Basin Texas, 1970: U.S. Geological Survey OpenFile Report, 50 p.

Hampton, B.B., 1973, Annual compilation and analysis of hydrologic data for Pin Oak Creek, Trinity River Basin Texas, 1971: U.S. Geological Survey Open-File Report, $18 \mathrm{p}$.

Hampton, B.B., 1974, Hydrologic data for Pin Oak Creek, Trinity River Basin Texas, 1972: U.S. Geological Survey Open-File Report, 26 p.

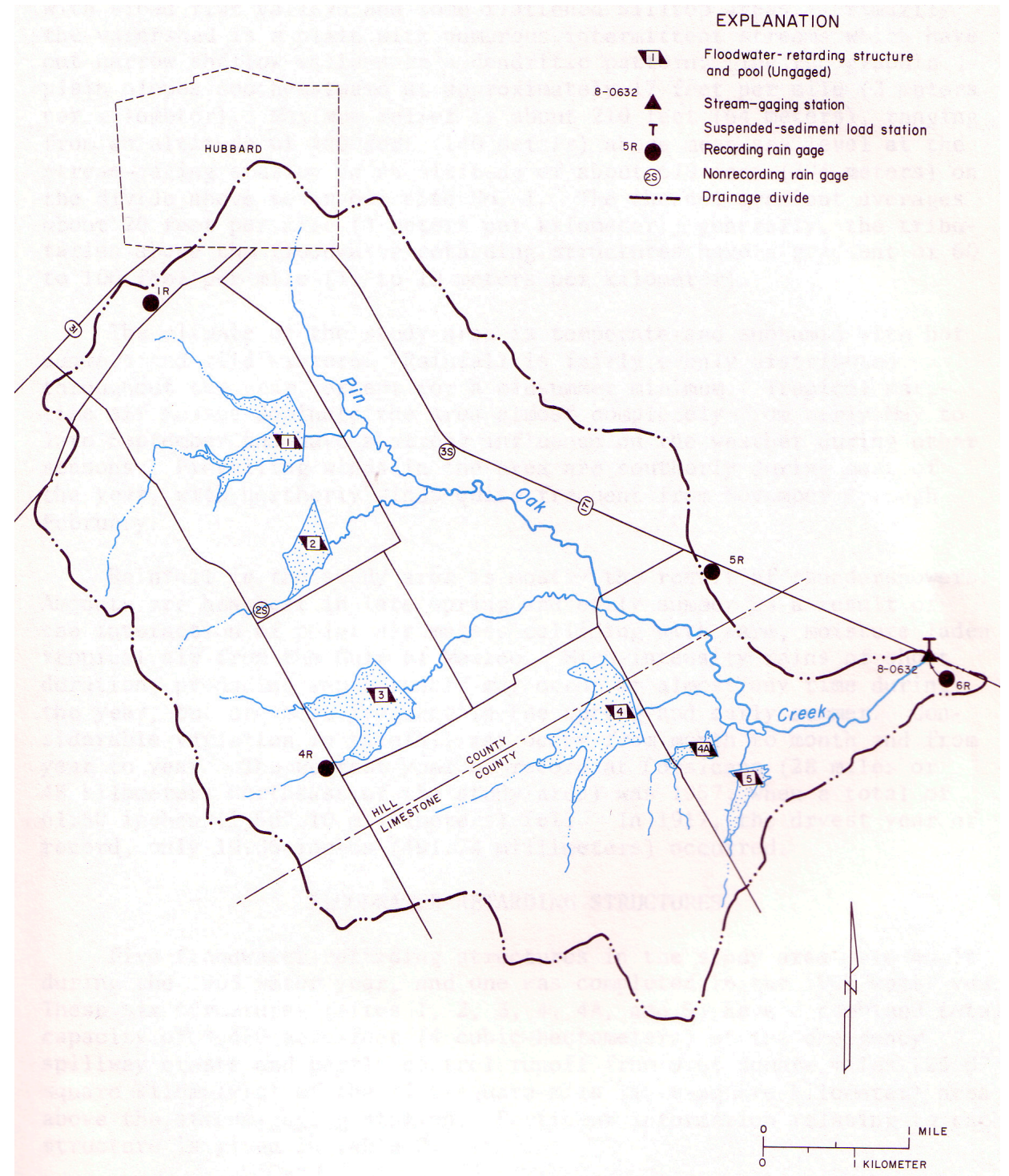

Figure 29. Location map for sma 11 rura 1 sheds database module for Pin Oak Creek watershed adopted from Hampton, 1974. 
This page intentionally left blank. 